\title{
Using Line Shapes to Discriminate between Binding Mechanisms for the $X(3872)$
}

\author{
Pierre Artoisenet, Eric Braaten, and Daekyoung Kang \\ Physics Department, Ohio State University, Columbus, Ohio 43210, USA
}

(Dated: November 20, 2018)

\begin{abstract}
We construct line shapes for the $X(3872)$ that generalize the Flatté and zero-range line shapes that have been considered previously. These line shapes are associated with scattering amplitudes that are exactly unitary for real values of the interaction parameters and can be derived from a renormalizable quantum field theory. The new line shapes can be used to discriminate between the alternative binding mechanisms in which the $X(3872)$ is generated either dynamically by charm meson interactions or by a resonance near the $D^{* 0} \bar{D}^{0}$ threshold. If the resonance is identified with the $\mathrm{P}$-wave charmonium state $\chi_{c 1}^{\prime}$, the interaction parameters can be constrained by using charmonium phenomenology. We analyze data on the $X(3872)$ and also data from the Belle and Babar Collaborations on the invariant mass distribution of the charm mesons from the decay $B \rightarrow K+D^{* 0} \bar{D}^{0}$ up to $4000 \mathrm{MeV}$. Our analysis is compatible with the mechanism for the $X(3872)$ being either a fine-tuning of charm meson interactions or the fine-tuning of the $\chi_{c 1}^{\prime}$ to the $D^{* 0} \bar{D}^{0}$ threshold. In particular, the data do not exclude a separate $\chi_{c 1}^{\prime}$ resonance between the $D^{*+} D^{-}$ threshold and $4000 \mathrm{MeV}$.
\end{abstract}

PACS numbers: 12.38.-t, 12.39.St, 13.20.Gd, 14.40.Rt 


\section{INTRODUCTION}

The discovery of the $X(3872)$ resonance by the Belle Collaboration in 2003 [1] marked the beginning of a new era in charmonium spectroscopy. About a dozen new $c \bar{c}$ mesons above the open charm threshold have been discovered and many of them have properties that seem incompatible with their identification as conventional charmonium states. This presents a serious challenge to our understanding of the $c \bar{c}$ sector of QCD. Of all the new $c \bar{c}$ mesons, the $X(3872)$ is the one for which the most experimental information is available. The preponderance of this information implies that the $X(3872)$ is a charm meson molecule whose constituents are a superposition of $D^{* 0} \bar{D}^{0}$ and $D^{0} \bar{D}^{* 0}$. The structure of this molecule is remarkable, with the charm mesons almost always very well separated. However this identification of the $X(3872)$ is not universally accepted within the field. The leading alternatives are the ${ }^{3} P_{1}$ charmonium state $\chi_{c 1}(2 P)$ or a compact tetraquark $c \bar{c}$ meson. Even among those who lean toward its identification as a charm meson molecule, the remarkable structure of the $X(3872)$ is not universally appreciated.

The only experimental information that is necessary to make the identification of the $X(3872)$ as a loosely-bound charm-meson molecule is the determination of its quantum numbers and the measurements of its mass. The quantum numbers of the $X(3872)$ can be inferred to be $1^{++}$by combining the following information:

- the observation of its decay into $J / \psi \gamma[2,3]$ or $\psi(2 S) \gamma[4]$, which implies that it is even under charge conjugation,

- analyses of the momentum distributions from its decay into $J / \psi \pi^{+} \pi^{-}$, which imply that its spin and parity are $1^{+}$or $2^{-}[5,6]$,

- either the observation of its decays into $D^{0} \bar{D}^{0} \pi^{0}[7]$, which disfavors $2^{-}$because of angular-momentum suppression, or the observation of its decay into $\psi(2 S) \gamma[4]$, which disfavors $2^{-}$because of multipole suppression.

A recent analysis of decays into $J / \psi \pi^{+} \pi^{-} \pi^{0}$ by the Babar Collaboration favors the quantum numbers $2^{-+}$, but does not exclude $1^{++}[8]$. In the absence of definitive evidence to the contrary, we will assume that the quantum numbers of the $X(3872)$ are $1^{++}$.

The mass of the $X(3872)$ can be determined most directly by measurements in the $J / \psi \pi^{+} \pi^{-}$decay channel. As pointed out in Ref. [9], measurements in the $D^{0} \bar{D}^{0} \pi^{0}$ decay channel are biased by the associated threshold enhancement just above the $D^{* 0} \bar{D}^{0}$ threshold. As pointed out in Ref. [10], measurements in the $D^{* 0} \bar{D}^{0}$ decay channel are biased by the analysis procedure in which $D^{0} \pi^{0}$ with invariant mass near the mass of $D^{* 0}$ is constrained to have invariant mass exactly equal to $M_{* 0}$. This procedure assigns an energy above the $D^{* 0} \bar{D}^{0}$ threshold to a $D^{0} \bar{D}^{0} \pi^{0}$ event whose energy is below the threshold. Using the most recent measurements of the mass of the $X(3872)$ in the $J / \psi \pi^{+} \pi^{-}$decay channel by the Belle, CDF, Babar, and D0 Collaborations [11-14], the combined average for the position of the $X(3872)$ resonance relative to the $D^{* 0} \bar{D}^{0}$ threshold is

$$
M_{X}-\left(M_{* 0}+M_{0}\right)=-0.42 \pm 0.39 \mathrm{MeV}
$$

where $M_{* 0}$ and $M_{0}$ are the masses of $D^{* 0}$ and $D^{0}$.

The reason the quantum numbers $1^{++}$and the measurement in Eq. (1) are sufficient to determine the nature of the $X(3872)$ is that quantum mechanics implies that an S-wave 
resonance whose energy is sufficiently close to the threshold has universal properties that are determined by its energy. If the energy is below the threshold, the state is a bound molecule consisting of pairs of particles that are almost always very well separated. One of the universal predictions is a relation between the mean-square separation of the constituents and the binding energy $E_{X}:\left\langle r^{2}\right\rangle_{X}=\left(4 \mu E_{X}\right)^{-1}$, where $\mu$ is the reduced mass. In the case of the $X(3872)$, the quantum numbers $1^{++}$imply that there is an S-wave coupling to $D^{* 0} \bar{D}^{0}$. The tiny energy relative to the $D^{* 0} \bar{D}^{0}$ threshold implies that it is a resonant coupling. Thus the $X(3872)$ is an S-wave threshold resonance. The binding energy given by Eq. (1) implies that the root-mean-square separation of the charm mesons in the $X(3872)$ is $\left\langle r^{2}\right\rangle_{X}^{1 / 2}=4.9_{-1.3}^{+13.4} \mathrm{fm}$. This huge separation of the charm mesons makes the $X(3872)$ a truly remarkable hadron.

One should distinguish between the nature of the $X(3872)$, which is a weakly-bound charm meson molecule, and its origin, which has to do with the binding mechanism for the molecule. Identifying the origin of the $X(3872)$ is crucial to understanding its implications for the other new $c \bar{c}$ mesons above the $D \bar{D}$ threshold. Universality is a double-edged sword. While it allows the nature of the $X(3872)$ to be determined unambiguously from limited experimental information, universality makes it more difficult to identify the origin of the state. There are two primary candidates for the binding mechanism of the $X(3872)$ :

- dynamical. The $X(3872)$ could be generated dynamically by the interactions between the charm mesons. The interaction between $D^{* 0}$ and $\bar{D}^{0}$ in the isospin- $01^{++}$channel could be tuned to near the critical strength for the formation of a bound state.

- $\underline{\text { resonance. }}$ The $X(3872)$ could be generated by an isospin-0 resonance whose energy is tuned to near the $D^{* 0} \bar{D}^{0}$ threshold. An obvious candidate for the resonance is the $\chi_{c 1}(2 P)$, but it could also be any other type of $c \bar{c}$ meson with the appropriate quantum numbers, such as a compact tetraquark.

One set of clues to the origin of the $X(3872)$ is its decay pattern. There are 6 decay modes that have been observed thus far: $J / \psi \pi^{+} \pi^{-}, J / \psi \pi^{+} \pi^{-} \pi^{0}[3], J / \psi \gamma[3,4], \psi(2 S) \gamma[4]$ $D^{0} \bar{D}^{0} \pi^{0}[7]$, and $D^{0} \bar{D}^{0} \gamma[15,16]$. In order to exploit this information, one would need to understand the pattern of branching fractions that follow from each of the binding mechanisms. There is a well-developed phenomenology for decays of charmonium states, but there is no corresponding phenomenology for decays of compact tetraquark $c \bar{c}$ mesons or for the inelastic scattering of charm meson pairs. This makes it difficult to constrain the origin of the $X(3872)$ using the observed branching ratios.

Another class of observables that can provide clues to the origin of the $X(3872)$ is the energy distribution or line shape in specific decay channels. At energies much less than $8 \mathrm{MeV}$ from the $D^{* 0} \bar{D}^{0}$ threshold, the line shapes in these and other channels are universal in the sense that they are determined only by the binding energy and width of the $X(3872)$ resonance [9]. The universal line shapes in the $D^{0} \bar{D}^{0} \pi^{0}$ and $D^{0} \bar{D}^{0} \gamma$ channels, which receive contributions from the decay of a constituent $D^{* 0}$ or $\bar{D}^{* 0}$, are different from those in other decay channels, such as $J / \psi \pi^{+} \pi^{-}$. Braaten and Lu presented simple analytic expressions for the universal line shapes that take into account the width of the $D^{* 0}$ and inelastic scattering channels for $D^{* 0} \bar{D}^{0}[9]$. The accuracy of these line shapes in the $D^{* 0} \bar{D}^{0}$ threshold region was verified in Ref. [17].

There have been several theoretical analyses of the line shapes for $X(3872)$ produced by the decays $B \rightarrow K+X$. The specific decay channels for which the line shapes have been 
measured by the Belle and Babar collaborations are $J / \psi \pi^{+} \pi^{-}[11-14,18,19], D^{0} \bar{D}^{0} \pi^{0}[7]$, and $D^{* 0} \bar{D}^{0}[15,16]$. Hanhart et al. used a Flatté parameterization of the line shapes to analyze the data on the $J / \psi \pi^{+} \pi^{-}$and $D^{0} \bar{D}^{0} \pi^{0}$ channels [20]. They concluded that the data favored the $X(3872)$ being a virtual state with energy above the $D^{* 0} \bar{D}^{0}$ threshold. Their analysis was biased towards such a result, because they assumed that the line shape in $D^{0} \bar{D}^{0} \pi^{0}$ vanishes below the $D^{* 0} \bar{D}^{0}$ threshold, thus ignoring any contributions from a resonance below the $D^{* 0} \bar{D}^{0}$ threshold. Braaten and Lu used the universal line shapes to analyze data on the $J / \psi \pi^{+} \pi^{-}$and $D^{0} \bar{D}^{0} \pi^{0}$ channels [9]. They concluded that the data favored the $X(3872)$ being a bound state with energy below the $D^{* 0} \bar{D}^{0}$ threshold. Zhang, Meng, and Zheng [21] followed Ref. [20] in using the Flatté line shapes and ignoring $D^{0} \bar{D}^{0} \pi^{0}$ and $D^{0} \bar{D}^{0} \gamma$ events from a resonance below the $D^{* 0} \bar{D}^{0}$ threshold. They concluded that $X(3872)$ can be identified as a ${ }^{3} P_{1}$ charmonium state that is strongly distorted by coupledchannel effects. Braaten and Stapleton used the universal line shapes to analyze data on the $J / \psi \pi^{+} \pi^{-}$and $D^{* 0} \bar{D}^{0}$ channels [22]. They pointed out that the analysis procedure for $D^{* 0} \bar{D}^{0}$ in Refs. $[15,16]$ assigns an energy above the $D^{* 0} \bar{D}^{0}$ threshold to a $D^{0} \bar{D}^{0} \pi^{0}$ or $D^{0} \bar{D}^{0} \gamma$ event from decay of a resonance just below the $D^{* 0} \bar{D}^{0}$ threshold. When this effect is taken into account, the analysis of the $D^{* 0} \bar{D}^{0}$ data favors the $X(3872)$ being a bound state with energy below the $D^{* 0} \bar{D}^{0}$ threshold. Kalashnikova and Nefediev [23] followed Ref. [20] in using the Flatté line shapes and ignoring $D^{0} \bar{D}^{0} \pi^{0}$ and $D^{0} \bar{D}^{0} \gamma$ events from a resonance below the $D^{* 0} \bar{D}^{0}$ threshold. They concluded that the Babar data prefers the $X(3872)$ to be a virtual state with a small ${ }^{3} P_{1}$ charmonium component while the Belle data prefers a bound state with a ${ }^{3} P_{1}$ charmonium component of about $30 \%$.

Since the lines shapes very close to the $D^{* 0} \bar{D}^{0}$ threshold are universal, information about the origin of the $X(3872)$ can only come from the energy distributions away from the threshold. The line shapes outside the universal region depend on the binding mechanism. The Flatté line shapes used in Refs. [20, 21, 23] can be derived from the assumption that the charm mesons scatter only through their couplings to a resonance with isospin 0 . These would be the appropriate line shapes if the $X(3872)$ arises from a tuning of the energy of a resonance. Alternative line shapes have been derived by Braaten and Lu under the assumption that the charm mesons scatter through zero-range interactions between the coupled channels consisting of neutral charm mesons and charged charm mesons [10]. These would be the appropriate line shapes if the $X(3872)$ arises dynamically from interactions between the charm mesons. The zero-range line shapes in the $J / \psi \pi^{+} \pi^{-}$and $J / \psi \pi^{+} \pi^{-} \pi^{0}$ channels had been considered previously by Voloshin [24], but his results were incorrect because of conceptual errors related to the treatment of isospin symmetry [10].

In order to discriminate between the two binding mechanisms identified above, it is necessary to use line shapes that allow for either possibility. There are two criteria that we use as guiding principles in constructing the line shapes:

- unitarity. The line shapes should correspond to multichannel scattering amplitudes that are exactly unitary for real values of the interaction parameters. Complex deformations of the parameters can then be used to take into account effects of additional channels that are not treated explicitly.

- renormalizability. The scattering amplitudes should be derivable from a renormalizable local quantum field theory. This guarantees that sensitivity to physics at much higher energies can be absorbed into the interaction parameters.

In a local quantum field theory, the production of particles by a short-distance process can 
be represented by local operators. An advantage of a renormalizable field theory is that one can construct renormalized operators whose matrix elements are insensitive to physics at much higher energies. The line shapes from that production process are then determined by the interaction parameters and by the short-distance coefficients of those renormalized operators.

In this paper, we present a solution to the coupled-channel problem for pairs of neutral charm mesons $D^{* 0} \bar{D}^{0}$ and $D^{0} \bar{D}^{* 0}$ and pairs of charged charm mesons $D^{*+} D^{-}$and $D^{+} D^{*-}$ that scatter through zero-range interactions and also through an isospin-0 resonance. The resulting scattering amplitudes satisfy the conditions of unitarity and renormalizability. They depend on 4 interaction parameters and reduce to the Flatté scattering amplitudes of Ref. [20] and to the zero-range scattering amplitudes of Ref. [10] in the appropriate limits. We also solve the renormalization problem for the local operators that represent the production at short distances of pairs of neutral charm mesons, pairs of charged charm mesons, and the resonance. The line shapes for $X(3872)$ produced by the decays $B \rightarrow K+X$ depend on the interaction parameters and on the short-distance coefficients associated with the $B \rightarrow K$ transition. We assume that the resonance is the $\chi_{c 1}(2 P)$ and we include constraints on the interaction parameters and on the $B \rightarrow K$ short-distance coefficients from charmonium phenomenology. We use our line shapes to analyze data on the $X(3872)$ and data from the Belle and Babar collaborations on the production of $D^{* 0} \bar{D}^{0}$ above the threshold up to $4000 \mathrm{MeV}$. We try to determine whether the data can discriminate between the two mechanisms for generating the $X(3872)$ that were described above.

We begin in Section II by establishing our notation. In Section III, we summarize the zero-range and Flatté scattering amplitudes that have been used in previous analyses and we present more general scattering amplitudes that include both as special cases. In Section IV, we present experimental and phenomenological constraints on the interaction parameters in the low-energy scattering amplitudes. In Section V, we express the line shapes of the $X(3872)$ from $B$ meson decays in terms of short-distance coefficients associated with the $B \rightarrow K$ transition. In Section VI, we present experimental and phenomenological constraints on the short-distance coefficients. In Section VII, we analyze data on $X(3872)$ and data on $B \rightarrow K+D^{* 0} \bar{D}^{0}$ to see whether they can discriminate between the two binding mechanisms. We summarize our results in Section VIII. In an Appendix, we present the quantum field theory formulation of the problem of charm mesons that scatter through both zero-range interactions and coupling to a resonance. We determine the renormalization of the interaction parameters in the scattering amplitudes. We also solve the renormalization problem for the local operators that create the charm meson pairs and the resonance.

\section{NOTATION}

The standard isospin multiplets for the charm mesons are $\left(-D^{+}, D^{0}\right),\left(\bar{D}^{0}, D^{-}\right)$, $\left(-D^{*+}, D^{* 0}\right)$, and $\left(\bar{D}^{* 0}, D^{*-}\right)$, where the first and second states are the upper and lower components of the multiplet, respectively. The most natural charge-conjugation phase conventions are $C D^{0}=+\bar{D}^{0}$ and $C D^{* 0}=-\bar{D}^{* 0}$. The $D^{*} \bar{D}$ channels with charge conjugation quantum number $C=+$ are then $[25]$

$$
\begin{aligned}
\left(D^{* 0} \bar{D}^{0}\right)_{+} & =\frac{1}{\sqrt{2}}\left(D^{* 0} \bar{D}^{0}-D^{0} \bar{D}^{* 0}\right) \\
\left(D^{*+} D^{-}\right)_{+} & =-\frac{1}{\sqrt{2}}\left(D^{*+} D^{-}-D^{+} D^{*-}\right) .
\end{aligned}
$$


We will refer to $\left(D^{* 0} \bar{D}^{0}\right)_{+}$and $\left(D^{*+} D^{-}\right)_{+}$as the neutral and charged channels, respectively.

We use concise notation for the masses and widths of the charm mesons. We denote the masses of $D^{0}, D^{+}, D^{* 0}$, and $D^{*+}$ by $M_{0}, M_{1}, M_{* 0}$, and $M_{* 1}$, respectively. (The numerical subscript is the absolute value of the electric charge of the meson.) The reduced mass for $D^{* 0} \bar{D}^{0}$ is $\mu=966.5 \mathrm{MeV}$. The reduced mass for $D^{*+} D^{-}$is larger by about $0.3 \%$, but we will ignore this difference. We measure energies relative to the $D^{* 0} \bar{D}^{0}$ threshold at $M_{* 0}+M_{0}=3871.9 \mathrm{MeV}$. The energy splitting between the $D^{*+} D^{-}$and $D^{* 0} \bar{D}^{0}$ thresholds is

$$
\nu_{11} \equiv\left(M_{* 1}+M_{1}\right)-\left(M_{* 0}+M_{0}\right)=8.1 \mathrm{MeV} .
$$

The corresponding momentum scale is

$$
\kappa_{11} \equiv \sqrt{2 \mu \nu_{11}}=125 \mathrm{MeV}
$$

The total width of the $D^{*+}$ is measured and the total width of the $D^{* 0}$ can be predicted from measurements of $D^{*}$ decays and isospin symmetry [9]. We denote these widths by $\Gamma_{* 1}$ and $\Gamma_{* 0}$, respectively. The PDG value for $\Gamma_{* 1}[26]$ and the predicted value for $\Gamma_{* 0}$ are

$$
\begin{aligned}
& \Gamma_{* 1}=96 \pm 22 \mathrm{keV}, \\
& \Gamma_{* 0}=66 \pm 15 \mathrm{keV} .
\end{aligned}
$$

The effects of decays of the $D^{* 0}$ and $D^{*+}$ on charm meson scattering can be partially taken into account through energy-dependent widths. In Ref. [9], energy-dependent widths $\Gamma_{* 0}(E)$ and $\Gamma_{* 1}(E)$ that depend on the energy $E$ of the pair of charm mesons in their center-of-mass frame were defined by scaling the physical partial widths for the decays $D^{*} \rightarrow D \pi$. The use of these energy-dependent widths above the $D^{*} \bar{D}$ thresholds was an error. If the energy is above the $D^{* 0} \bar{D}^{0}$ threshold at $E=0$, the appropriate width for the $D^{* 0}$ is the physical width $\Gamma_{* 0}$. If the energy is above the $D^{*+} D^{-}$threshold at $E=\nu_{11}$, the appropriate width for the $D^{*+}$ is the physical width $\Gamma_{* 1}$. Below the $D^{*} \bar{D}$ thresholds, the energy-dependent widths of Ref. [9] give thresholds at the correct energies for the 3-body $D \bar{D} \pi$ states. However, as pointed out in [17], they do not give the correct energy dependence just above these thresholds. Moreover, the effects of the energy-dependent widths on the line shape of the $X(3872)$ are numerically small. We will therefore ignore any energy dependence of the widths in this paper.

\section{LOW-ENERGY $D^{*} \bar{D}$ SCATTERING}

In this section, we discuss the low-energy scattering of the charm mesons $D^{*}$ and $\bar{D}$. We first summarize the universal scattering amplitude of Ref. [9], which takes into account the large scattering length in the neutral channel. We then describe the coupled-channel scattering amplitudes of Ref. [10], which take into account zero-range scattering in the neutral and charged channels, and the Flatté scattering amplitudes introduced in Ref. [20], which take into account scattering through a resonance. Finally, we present more general scattering amplitudes that allow for scattering both through a resonance and through zerorange interactions. 


\section{A. Universal scattering amplitude}

We first consider neutral charm meson pairs with scattering only in the channel $\left(D^{* 0} \bar{D}^{0}\right)_{+}$ defined in Eq. (2a). The transition amplitude $\mathcal{A}(E)$ for the scattering of nonrelativistically normalized charm meson pairs can be written in the form

$$
\mathcal{A}(E)=\frac{2 \pi}{\mu} f(E)
$$

where $f(E)$ is the conventional nonrelativistic scattering amplitude expressed as a function of the total energy $E$ of the charm mesons in the center-of-mass frame. We measure $E$ relative to the $D^{* 0} \bar{D}^{0}$ threshold. The universal scattering amplitude for an S-wave threshold resonance is

$$
f(E) \equiv \frac{1}{-\gamma+\kappa(E)},
$$

where $\kappa(E)=(-2 \mu E-i \varepsilon)^{1 / 2}$ and $\gamma$ is the inverse scattering length. If $\gamma$ is a real parameter, $f(E)$ satisfies the constraints of unitarity for a single-channel system exactly. This universal scattering amplitude can be derived from a renormalizable nonrelativistic quantum field theory with a contact interaction in a single scattering channel.

The imaginary part of $f(E)$ can be interpreted as a spectral function for the resonance. The spectral function associated with the scattering amplitude in Eq. (7) is

$$
\operatorname{Im} f(E)=|f(E)|^{2}[\operatorname{Im} \gamma-\operatorname{Im} \kappa(E)] .
$$

If $\gamma$ is a real parameter, the spectral function reduces to

$$
\operatorname{Im} f(E)=\theta(E)|f(E)|^{2} \sqrt{2 \mu E}+\theta(\gamma) \frac{\pi \gamma}{\mu} \delta\left(E+\gamma^{2} /(2 \mu)\right)
$$

There is a threshold enhancement associated with production of $D^{* 0} \bar{D}^{0}$ and $D^{0} \bar{D}^{* 0}$ with a peak at $E=+\gamma^{2} /(2 \mu)$. If $\gamma>0$, there is also a delta function contribution at $E=-\gamma^{2} /(2 \mu)$ associated with a bound state with binding energy $\gamma^{2} /(2 \mu)$.

Scattering in the $\left(D^{* 0} \bar{D}^{0}\right)+$ channel cannot be exactly unitary, because the $D^{* 0}$ has a nonzero width and because the charm meson pair has inelastic scattering channels. Following Ref. [9], the dominant effects of decays of the $D^{* 0}$ can be taken into account by replacing $\kappa(E)$ by

$$
\kappa(E)=\sqrt{-2 \mu E-i \mu \Gamma_{* 0}},
$$

where $\Gamma_{* 0}$ is the width of the $D^{* 0}$ given in Eq. (5b). Following Ref. [9], the effects of inelastic scattering channels for the charm-meson pair other than $D^{0} \bar{D}^{0} \pi^{0}$ and $D^{0} \bar{D}^{0} \gamma$ can be taken into account by making $\gamma$ a complex parameter with a positive imaginary part. In the expression for the imaginary part of $f(E)$ in Eq. (8), the term proportional to $\operatorname{Im} \kappa(E)$ is the contribution from channels whose ultimate final states are $\left(D^{0} \bar{D}^{0} \pi^{0}, D^{0} \bar{D}^{0} \gamma\right)$, including $D^{* 0} \bar{D}^{0}$ and $D^{0} \bar{D}^{* 0}$. The term proportional to $\operatorname{Im} \gamma$ is the contribution from all other channels, including $J / \psi \pi^{+} \pi^{-}$. The $\operatorname{Im} \kappa(E)$ term has a threshold enhancement just above the $D^{* 0} \bar{D}^{0}$ threshold. If $\operatorname{Re}(\gamma)>0$, both terms have a resonant peak just below the threshold that can be identified with the $X(3872)$. Its position and width are determined by the pole of the scattering amplitude $f(E)$ in Eq. (7). The complex energy of the pole can be expressed as

$$
E_{\text {pole }}=-\gamma^{2} /(2 \mu)-i \Gamma_{* 0} / 2 .
$$

Quantitative constraints on the real and imaginary parts of $\gamma$ are presented in Section IV A. 


\section{B. Zero-Range scattering amplitudes}

In Ref. [10], the universal scattering amplitude of Section III A was generalized to the case of coupled channels $\left(D^{* 0} \bar{D}^{0}\right)_{+}$and $\left(D^{*+} D^{-}\right)_{+}$defined by Eqs. (2) that scatter through zero-range interactions.

\section{General case}

We label the two channels $\left(D^{* 0} \bar{D}^{0}\right)_{+}$and $\left(D^{*+} D^{-}\right)_{+}$by the indices 0 and 1 , respectively. The transition amplitudes $\mathcal{A}_{i j}(E)$ among these two channels define scattering amplitudes $f_{i j}(E)$ that depend on the total energy $E$ relative to the $D^{* 0} \bar{D}^{0}$ threshold:

$$
\mathcal{A}_{i j}(E)=\frac{2 \pi}{\mu} f_{i j}(E)
$$

If the charm mesons scatter through a zero-range interaction, the inverse of the $2 \times 2$ matrix of scattering amplitudes has the form

$$
f(E)^{-1}=-\Lambda^{-1}+K(E),
$$

where $\Lambda$ is $2 \times 2$ symmetric matrix. The dependence on the energy $E$ is in the diagonal matrix

$$
K(E)=\left(\begin{array}{cc}
\kappa(E) & 0 \\
0 & \kappa_{1}(E)
\end{array}\right)
$$

whose diagonal entries are $\kappa(E)=(-2 \mu E-i \varepsilon)^{1 / 2}$ and $\kappa_{1}(E)=\left(-2 \mu\left(E-\nu_{11}\right)-i \varepsilon\right)^{1 / 2}$. If the three interaction parameters $\Lambda_{00}, \Lambda_{01}$, and $\Lambda_{11}$ are all real valued, the scattering amplitudes $f_{i j}(E)$ satisfy the constraints of unitarity for this two-channel system exactly. They can be derived from a renormalizable nonrelativistic quantum field theory with zerorange interactions.

\section{Isospin symmetry}

The approximate isospin symmetry of QCD reduces the three interaction parameters $\Lambda_{i j}$ to two independent parameters $\gamma_{0}$ and $\gamma_{1}$ :

$$
\Lambda=\left(1 / \gamma_{0}\right) \mathcal{P}_{0}+\left(1 / \gamma_{1}\right) \mathcal{P}_{1}
$$

where the matrices $\mathcal{P}_{0}$ and $\mathcal{P}_{1}$ are projectors onto the isospin 0 and 1 channels:

$$
\begin{aligned}
& \mathcal{P}_{0}=\frac{1}{2}\left(\begin{array}{rr}
1 & -1 \\
-1 & 1
\end{array}\right), \\
& \mathcal{P}_{1}=\frac{1}{2}\left(\begin{array}{ll}
1 & 1 \\
1 & 1
\end{array}\right) .
\end{aligned}
$$


The scattering amplitudes obtained by inverting Eq. (13) reduce to

$$
\begin{aligned}
& f_{00}(E)=\frac{-\left(\gamma_{0}+\gamma_{1}\right)+2 \kappa_{1}(E)}{D_{0}(E)} \\
& f_{01}(E)=\frac{\gamma_{1}-\gamma_{0}}{D_{0}(E)} \\
& f_{11}(E)=\frac{-\left(\gamma_{0}+\gamma_{1}\right)+2 \kappa(E)}{D_{0}(E)}
\end{aligned}
$$

where the denominator is

$$
D_{0}(E)=2 \gamma_{1} \gamma_{0}-\left(\gamma_{1}+\gamma_{0}\right)\left[\kappa_{1}(E)+\kappa(E)\right]+2 \kappa_{1}(E) \kappa(E) .
$$

At energies $E$ far from the charm meson thresholds at 0 and $\nu_{11}$, the difference between $\kappa_{1}(E)$ and $\kappa(E)$ can be neglected and the isospin symmetry becomes exact. By considering this limit, we can identify $\gamma_{0}$ and $\gamma_{1}$ as the isoscalar and isovector inverse scattering lengths, respectively. We will refer to the model defined by the scattering amplitudes in Eqs. (17) as the Zero-Range model.

\section{Optical theorem}

Scattering in the $\left(D^{* 0} \bar{D}^{0}\right)_{+}$and $\left(D^{*+} D^{-}\right)_{+}$channels cannot be exactly unitary, because the $D^{* 0}$ and $D^{*+}$ have nonzero widths and because the charm meson pairs have inelastic scattering channels. Following Ref. [10], the dominant effects of decays of $D^{* 0}$ and $D^{*+}$ can be taken into account by replacing $\kappa(E)$ by the expression in Eq. (10) and by replacing $\kappa_{1}(E)$ by

$$
\kappa_{1}(E)=\sqrt{-2 \mu\left(E-\nu_{11}\right)-i \mu \Gamma_{* 1}}
$$

where $\Gamma_{* 1}$ is the width of the $D^{*+}$ given in Eq. (5a). The contribution of $\Gamma_{* 1}$ to the imaginary part of $\kappa_{1}(E)$ is only important near the $D^{*+} D^{-}$threshold. Near the $D^{* 0} \bar{D}^{0}$ threshold, $\kappa_{1}(E)$ is well approximated by the real quantity $\kappa_{11}=125 \mathrm{MeV}$. Following Ref. [10], the effects of inelastic scattering channels other than $D \bar{D} \pi$ and $D \bar{D} \gamma$ can be taken into account by taking $\gamma_{0}$ and $\gamma_{1}$ to be complex parameters with positive imaginary parts.

The imaginary parts of the scattering amplitudes in Eq. (17) can be expressed in forms that are consistent with the Cutkosky cutting rules:

$$
\begin{aligned}
\operatorname{Im} f_{i j}(E)= & \operatorname{Im} \gamma_{0} \sum_{k, l} f_{i k}(E) \mathcal{P}_{0, k l} f_{l j}^{*}(E)+\operatorname{Im} \gamma_{1} \sum_{k, l} f_{i k}(E) \mathcal{P}_{1, k l} f_{l j}^{*}(E) \\
& -\operatorname{Im} \kappa(E) f_{i 0}(E) f_{0 j}^{*}(E)-\operatorname{Im} \kappa_{1}(E) f_{i 1}(E) f_{1 j}^{*}(E)
\end{aligned}
$$

The terms in Eq. (20) proportional to $\operatorname{Im} \kappa(E)$ and $\operatorname{Im} \kappa_{1}(E)$ are the contributions from channels whose ultimate final states are $(D \bar{D} \pi, D \bar{D} \gamma)$, including $D^{*} \bar{D}$ and $D \bar{D}^{*}$. The terms proportional to $\operatorname{Im} \gamma_{0}$ and $\operatorname{Im} \gamma_{1}$ correspond to other inelastic scattering channels with isospin 0 and 1 , respectively.

\section{4. $D^{* 0} \bar{D}^{0}$ threshold region}

The interaction parameters $\gamma_{0}$ and $\gamma_{1}$ can be tuned so that there is a bound state just below the $D^{* 0} \bar{D}^{0}$ threshold that can be identified with the $X(3872)$. The scattering amplitudes 
$f_{i j}(E)$ have a pole at a complex energy $E_{\text {pole }}$ that satisfies Eq. (11) with $\gamma \equiv \kappa\left(E_{\text {pole }}\right)$. If the tiny difference between $\kappa_{1}\left(E_{\text {pole }}\right)$ and $\kappa_{11}$ is neglected, the vanishing of the denominator $D_{0}(E)$ in Eq. (18) reduces to a linear equation for $\gamma$, whose solution is

$$
\gamma=\frac{2 \gamma_{1} \gamma_{0}-\left(\gamma_{1}+\gamma_{0}\right) \kappa_{11}}{\gamma_{1}+\gamma_{0}-2 \kappa_{11}}
$$

An inverse scattering length $\gamma$ that is small compared to $\kappa_{11}$ requires a fine-tuning of $\gamma_{0}$ and $\gamma_{1}$ so that $2 \gamma_{1} \gamma_{0} \approx\left(\gamma_{1}+\gamma_{0}\right) \kappa_{11}$. If $\left|\gamma_{1}\right| \gg \kappa_{11}, \gamma_{0}$ must be fine-tuned to near $\kappa_{11} / 2 \approx+63 \mathrm{MeV}$. Since line shapes near the $D^{* 0} D^{0}$ threshold are extremely sensitive to $\gamma$, it is advantageous to take $\gamma$ to be one of the independent interaction parameters. This can be accomplished by eliminating $\gamma_{0}$ in favor of $\gamma$ using

$$
\gamma_{0}=\frac{\gamma_{1} \kappa_{11}+\left(\gamma_{1}-2 \kappa_{11}\right) \gamma}{\left(2 \gamma_{1}-\kappa_{11}\right)-\gamma}
$$

which follows from Eq. (21).

The scattering amplitudes $f_{i j}(E)$ in Eqs. (17) all have poles in the energy variable $\kappa(E)$ at $\kappa(E)=\gamma$. The residue of the pole of $f_{i j}(E)$ has the form $Z_{i}^{1 / 2} Z_{j}^{1 / 2}$. The ratio of the residue factors is

$$
\frac{Z_{1}^{1 / 2}}{Z_{0}^{1 / 2}}=-\frac{\gamma_{1}-\gamma}{\gamma_{1}-\kappa_{11}} .
$$

If the tiny difference between $\kappa_{1}\left(E_{\text {pole }}\right)$ and $\kappa_{11}$ is neglected, the residue $Z_{0}$ is

$$
Z_{0}=\left(1+\frac{\left(\gamma_{1}-\gamma\right)^{2} \gamma}{\left(\gamma_{1}-\kappa_{11}\right)^{2} \kappa_{11}}\right)^{-1}
$$

The behavior of the elastic scattering amplitude $f_{00}(E)$ in the entire $D^{* 0} \bar{D}^{0}$ threshold region defined by $|E| \ll \nu_{11}$ is dominated by the pole at $\kappa(E)=\gamma$. It reduces in this region to $Z_{0} f(E)$, where $f(E)$ is the universal elastic scattering amplitude in Eq. (7). As $\gamma \rightarrow 0, Z_{0}$ approaches 1.

\section{Flatté scattering amplitudes}

In Ref. [20], Hanhart, Nefediev, and Kalashnikova proposed Flatté line shapes for the $X(3872)$ resonance. The Flatté scattering amplitudes can be derived by assuming that the coupled channels $\left(D^{* 0} \bar{D}^{0}\right)_{+}$and $\left(D^{*+} D^{-}\right)_{+}$scatter only through their couplings to a resonance.

\section{Isospin symmetry}

If two channels scatter only through their couplings to an isospin- 0 resonance, the entries of the $2 \times 2$ matrix of scattering amplitude defined by Eq. (12) are

$$
f_{00}(E)=f_{11}(E)=-f_{01}(E)=f_{\text {Flatté }}(E)
$$


where $f_{\text {Flatté }}(E)$ is the Flatté scattering amplitude:

$$
f_{\text {Flatté }}(E) \equiv \frac{-g^{2} / 2}{E-\nu-\left(g^{2} / 2\right)\left[\kappa_{1}(E)+\kappa(E)\right]} .
$$

The threshold functions $\kappa(E)$ and $\kappa_{1}(E)$ are given in Eqs. (10) and (19). If we take $g$ to be real, the spectral function associated with the Flatté scattering amplitude in Eq. (26) is

$$
\operatorname{Im} f_{\text {Flatté }}(E)=\left|f_{\text {Flatté }}(E)\right|^{2}\left[-\frac{2}{g^{2}} \operatorname{Im} \nu-\operatorname{Im} \kappa_{1}(E)-\operatorname{Im} \kappa(E)\right] \text {. }
$$

The notation of Ref. [20] can be obtained by the substitutions

$$
\begin{aligned}
g^{2} & \longrightarrow g \\
\nu & \longrightarrow E_{f}-i \Gamma(E) / 2 .
\end{aligned}
$$

In Ref. [20], the effects of the widths of the $D^{* 0}$ and $D^{*+}$ were not taken into account. Thus the expressions for $\kappa(E)$ and $\kappa_{1}(E)$ were Eq. (10) with $\Gamma_{* 0}=0$ and Eq. (19) with $\Gamma_{* 1}=0$. The authors of Ref. [20] did however allow for energy dependence in $\operatorname{Im} \nu$, as indicated by Eq. (28b).

\section{2. $D^{* 0} \bar{D}^{0}$ threshold region}

The interaction parameters $\nu$ and $g$ can be tuned so that there is a bound state just below the $D^{* 0} \bar{D}^{0}$ threshold that can be identified with the $X(3872)$. The scattering amplitude will have a pole at a complex energy $E_{\text {pole }}$ that satisfies Eq. (11) with $\gamma \equiv \kappa\left(E_{\text {pole }}\right)$. If the tiny difference between $\kappa_{1}\left(E_{\text {pole }}\right)$ and $\kappa_{11}$ is neglected, the vanishing of the denominator in Eq. (26) reduces to a quadratic equation for $\gamma$, one of whose solutions is

$$
\gamma=-\frac{1}{2} g^{2} \mu+\sqrt{\left(\frac{1}{2} g^{2} \mu\right)^{2}-\mu\left(2 \nu+g^{2} \kappa_{11}+i \Gamma_{* 0}\right)} .
$$

The residues of the poles of the scattering amplitudes $f_{i j}(E)$ at $\kappa(E)=\gamma$ are $Z_{i}^{1 / 2} Z_{j}^{1 / 2}$, where

$$
Z_{0}^{1 / 2}=-Z_{1}^{1 / 2}=\left(1+\frac{\gamma}{\kappa_{11}}+\frac{2 \gamma}{g^{2} \mu}\right)^{-1 / 2} .
$$

An inverse scattering length $\gamma$ that is small compared to $\kappa_{11}$ can be obtained by a fine-tuning of $\nu$ such that $\left|\nu+g^{2} \kappa_{11} / 2\right| \ll g^{4} \mu / 8, g^{2} \kappa_{11} / 2$. The solution for $\gamma$ in Eq. (29) then reduces to $\gamma \approx-\left(2 \nu+g^{2} \kappa_{11}+i \Gamma_{* 0}\right) / g^{2}$. In this case, the elastic scattering amplitude $f_{00}(E)$ reduces in the entire $D^{* 0} \bar{D}^{0}$ threshold region $|E| \ll \nu_{11}$ to $Z_{0} f(E)$, where $f(E)$ is the universal scattering amplitude $f(E)$ in Eq. (7).

An inverse scattering length $\gamma$ that is small compared to $\kappa_{11}$ can also be obtained by a double fine-tuning of $g$ and $\nu$ so that $g^{2} \mu \ll \kappa_{11}$ and $\mu|\nu| \ll \kappa_{11}^{2}$. In this case, the denominator in Eq. (26) has two zeroes near the $D^{* 0} \bar{D}^{0}$ threshold. The energy dependence of $f_{00}(E)$ in the $D^{* 0} \bar{D}^{0}$ threshold region is therefore more complicated than the universal scattering amplitude. 


\section{Resonance far above $D^{*} \bar{D}$ threshold}

Alternatively, the parameters $\nu$ and $g$ can be chosen so that, instead of having a pole just below the $D^{* 0} \bar{D}^{0}$ threshold, the Flatté scattering amplitude in Eq. (26) has a pole at an energy well above the $D^{*+} D^{-}$threshold. Such a pole could be associated with the charmonium state $\chi_{c 1}(2 P)$. If the difference between $\kappa_{1}(E)$ and $\kappa(E)$ is neglected, we get a quadratic equation for $\kappa$. The solution for the complex pole is

$$
E_{\chi}-i \Gamma_{\chi} / 2 \approx \nu-g^{4} \mu-i g^{2} \sqrt{2 \mu \nu-g^{4} \mu^{2}}
$$

If $\nu$ has a small imaginary part, the energy and width of the resonance are approximately

$$
\begin{aligned}
& E_{\chi} \approx \operatorname{Re} \nu-g^{4} \mu \\
& \Gamma_{\chi} \approx-2 \operatorname{Im} \nu+2 g^{2} \sqrt{2 \mu\left(\operatorname{Re} \nu-g^{4} \mu\right)+g^{4} \mu^{2}} .
\end{aligned}
$$

In the expression for the width, the term with the square root is the partial width for decays into $D^{*} \bar{D}$. The term proportional to $\operatorname{Im} \nu$ corresponds to all other decay channels. As the energy $E_{\chi}$ is increased by adjusting $\nu$, the width $\Gamma_{\chi}$ increases as $E_{\chi}^{1 / 2}$. This behavior is characteristic of an ordinary resonance. Near the resonance, the Flatté scattering amplitude in Eq. (26) can be approximated by

$$
f_{\text {Flatté }}(E) \approx\left(1-i \frac{g^{2} \mu}{\sqrt{2 \mu E_{\chi}-i \mu \Gamma_{\chi}}}\right)^{-1} f_{\mathrm{BW}}(E),
$$

where $f_{\mathrm{BW}}(E)$ is the Breit-Wigner scattering amplitude:

$$
f_{\mathrm{BW}}(E) \equiv \frac{-g^{2} / 2}{E-E_{\chi}+i \Gamma_{\chi} / 2}
$$

\section{Zero-Range+Resonance scattering amplitudes}

We now generalize the scattering amplitudes of Sections III B and III C to the case of coupled channels $\left(D^{* 0} \bar{D}^{0}\right)_{+}$and $\left(D^{*+} D^{-}\right)_{+}$that scatter through a resonance as well as through zero-range interactions. We will refer to the resonance channel as $\chi$.

\section{General case}

The transition amplitudes $\mathcal{A}_{i j}(E)$ in Eq. (12) define a $2 \times 2$ matrix of scattering amplitudes $f_{i j}(E)$. The expression in Eq. (13) for the inverse of that $2 \times 2$ matrix in the case of zerorange scattering can be generalized to one that also takes into account the coupling to a resonance:

$$
f(E)^{-1}=-\left(\Lambda+G \frac{1}{E-\nu} G^{T}\right)^{-1}+K(E),
$$

where $\Lambda$ is a $2 \times 2$ symmetric matrix, $G$ is a 2 -component column vector, and $K(E)$ is the diagonal matrix in Eq. (14). The propagator for this resonance is

$$
P(E)=\left[E-\nu+G^{T}\left(\Lambda-K^{-1}\right)^{-1} G\right]^{-1} .
$$


If the six interaction parameters $\Lambda_{00}, \Lambda_{01}, \Lambda_{11}, G_{0}, G_{1}$, and $\nu$ are all real valued and if $\Gamma_{* 0}$ and $\Gamma_{* 1}$ are set to 0 in the expressions for Eqs. (10) and (19), the amplitudes $f_{i j}(E)$ satisfy the constraints of unitarity exactly. The scattering amplitudes and the resonance propagator can be derived from a renormalizable nonrelativistic quantum field theory with two scattering channels and a resonance that interact only through contact interactions. The renormalization of this quantum field theory is described in the Appendix.

\section{Isospin symmetry}

The approximate isospin symmetry of QCD reduces the three interaction parameters in the matrix $\Lambda$ to two independent parameters $\gamma_{0}$ and $\gamma_{1}$ defined by Eq. (15). The assumption that the resonance has isospin 0 reduces the two interaction parameters in the column vector $G$ to a single parameter $g$ :

$$
G=\frac{g}{\sqrt{2}}\left(\begin{array}{r}
1 \\
-1
\end{array}\right)
$$

Thus the inverse matrix in Eq. (35) reduces to

$$
\left(\Lambda+G \frac{1}{E-\nu} G^{T}\right)^{-1}=\left(\frac{1}{\gamma_{0}}+\frac{g^{2}}{E-\nu}\right)^{-1} \mathcal{P}_{0}+\gamma_{1} \mathcal{P}_{1}
$$

The scattering amplitudes obtained by inverting the matrix in Eq. (35) can be obtained from those in Eq. (17) by replacing $\gamma_{0}$ by $\left[1 / \gamma_{0}+g^{2} /(E-\nu)\right]^{-1}$ :

$$
\begin{aligned}
& f_{00}(E)=\frac{\left[-\gamma_{1}-\gamma_{0}+2 \kappa_{1}(E)\right]\left(E-\nu+g^{2} \gamma_{0}\right)+g^{2} \gamma_{0}^{2}}{D(E)} \\
& f_{01}(E)=\frac{\left(\gamma_{1}-\gamma_{0}\right)\left(E-\nu+g^{2} \gamma_{0}\right)+g^{2} \gamma_{0}^{2}}{D(E)} \\
& f_{11}(E)=\frac{\left[-\gamma_{1}-\gamma_{0}+2 \kappa(E)\right]\left(E-\nu+g^{2} \gamma_{0}\right)+g^{2} \gamma_{0}^{2}}{D(E)}
\end{aligned}
$$

where the denominator is

$$
\begin{aligned}
D(E)= & {\left[2 \gamma_{1} \gamma_{0}-\left(\gamma_{1}+\gamma_{0}\right)\left[\kappa_{1}(E)+\kappa(E)\right]+2 \kappa_{1}(E) \kappa(E)\right]\left(E-\nu+g^{2} \gamma_{0}\right) } \\
& +g^{2} \gamma_{0}^{2}\left[-2 \gamma_{1}+\kappa_{1}(E)+\kappa(E)\right] .
\end{aligned}
$$

The resonance propagator in Eq. (36) is

$$
P(E)=\frac{2 \gamma_{1} \gamma_{0}-\left(\gamma_{1}+\gamma_{0}\right)\left[\kappa_{1}(E)+\kappa(E)\right]+2 \kappa_{1}(E) \kappa(E)}{D(E)} .
$$

At energies $E$ far from the charm meson thresholds at 0 and $\nu_{11}$, the difference between $\kappa_{1}(E)$ and $\kappa(E)$ can be neglected and the isospin symmetry becomes exact. The scattering amplitudes in Eqs. (39) and the resonance propagator in Eq. (41) depend on four independent interaction parameters: $\gamma_{0}, \gamma_{1}, g$, and $\nu$. We will refer to the model with the scattering amplitudes in Eqs. (39) and the resonance propagator in Eq. (41) as the Zero-Range+Resonance model. 


\section{Optical theorem}

The system consisting of the three channels $\left(D^{* 0} \bar{D}^{0}\right)_{+},\left(D^{*+} D^{-}\right)_{+}$, and $\chi$ cannot be exactly unitary, because the $D^{* 0}$ and $D^{*+}$ have nonzero widths, the charm meson pairs have inelastic scattering channels, and $\chi$ may have decay channels other than $D^{*} \bar{D}$ and $D \bar{D}^{*}$. We can take into account the dominant effects of decays of $D^{* 0}$ and $D^{*+}$ by replacing $\kappa(E)$ and $\kappa_{1}(E)$ by the expressions in Eqs. (10) and (19), respectively. We can take into account the effects of inelastic scattering channels other than $D \bar{D} \pi$ and $D \bar{D} \gamma$ by taking $\gamma_{0}$ and $\gamma_{1}$ to be complex parameters with positive imaginary parts. We can take into account the effects of decay channels for $\chi$ other than $D^{*} \bar{D}$ and $D \bar{D}^{*}$ by taking $\nu$ to be a complex parameter with a negative imaginary part. We choose $g^{2}$ to be a real parameter. The imaginary parts of the scattering amplitudes in Eq. (39) can be expressed in forms that are consistent with the Cutkosky cutting rules:

$$
\begin{aligned}
\operatorname{Im} f_{i j}(E)= & \left(\frac{|E-\nu|^{2}}{\left|E-\nu+g^{2} \gamma_{0}\right|^{2}} \operatorname{Im} \gamma_{0}-\frac{g^{2}\left|\gamma_{0}\right|^{2}}{\left|E-\nu+g^{2} \gamma_{0}\right|^{2}} \operatorname{Im} \nu\right) \sum_{k, l} f_{i k}(E) \mathcal{P}_{0, k l} f_{l j}^{*}(E) \\
& +\operatorname{Im} \gamma_{1} \sum_{k, l} f_{i k}(E) \mathcal{P}_{1, k l} f_{l j}^{*}(E) \\
& -\operatorname{Im} \kappa(E) f_{i 0}(E) f_{0 j}^{*}(E)-\operatorname{Im} \kappa_{1}(E) f_{i 1}(E) f_{1 j}^{*}(E) .
\end{aligned}
$$

The terms proportional to $\operatorname{Im} \kappa(E)$ and $\operatorname{Im} \kappa_{1}(E)$ are the contributions from channels whose ultimate final states are $(D \bar{D} \pi, D \bar{D} \gamma)$, including $D^{*} \bar{D}$ and $D \bar{D}^{*}$. The terms proportional to $\operatorname{Im} \gamma_{0}, \operatorname{Im} \gamma_{1}$, and $\operatorname{Im} \nu$ correspond to other inelastic $D^{*} \bar{D}$ scattering channels with isospin 0 ,

other inelastic $D^{*} \bar{D}$ scattering channels with isospin 1, and decay channels of $\chi$ with isospin 0 , respectively.

\section{Zero-range limit}

In the limits $g \rightarrow 0$ or $\nu \rightarrow \infty$, the resonance decouples and the scattering amplitudes for the Zero-Range+Resonance model in Eqs. (39) reduce to those for the Zero-Range model in Eqs. (17). The Zero-Range model is a good approximation if $|E-\nu| \gg g^{2}\left|\gamma_{0}\right|$. This energy region includes the $D^{* 0} \bar{D}^{0}$ threshold if $|\nu| \gg g^{2}\left|\gamma_{0}\right|$, in which case the condition on the energy reduces to $|E| \ll|\nu|$.

The Zero-Range model actually has a larger domain of validity if one allows for renormalization of the parameter $\gamma_{0}$. In the region $|E| \ll|\nu|,\left|\nu-g^{2} \gamma_{0}\right|$, the scattering amplitudes in Eqs. (39) reduce to those in Eqs. (17) with the substitution $\gamma_{0} \rightarrow \gamma_{0} \nu /\left(\nu-g^{2} \gamma_{0}\right)$.

\section{Flatté limit}

In the limits $\gamma_{0}, \gamma_{1} \rightarrow \infty$, scattering proceeds only through the resonance and the scattering amplitudes for the Zero-Range+Resonance model in Eqs. (39) reduce to the Flatté scattering amplitudes in Eqs. (25) and (26). The resonance propagator in Eq. (41) reduces in this limit to $P(E) \approx\left(-2 / g^{2}\right) f_{\text {Flatté }}(E)$. The Flatté model is a good approximation in the region $\left|\gamma_{1}\right| \gg \kappa_{11}$ if the energy satisfies $|E| \ll\left|\gamma_{1}\right|^{2} / \mu$ and $|E-\nu| \ll g^{2}\left|\gamma_{0}\right|, g^{2}\left|\gamma_{1}\right|$. This energy region includes the $D^{* 0} \bar{D}^{0}$ threshold if $|\nu| \ll g^{2}\left|\gamma_{0}\right|, g^{2}\left|\gamma_{1}\right|$. 
The Flatté model actually has a larger domain of validity if one allows for renormalization of the coupling constant $g$. If $\left|\gamma_{1}\right| \gg \kappa_{11}$ and if the energy satisfies $|E| \ll\left|\gamma_{1}\right|^{2} / \mu,\left|\nu-g^{2} \gamma_{0}\right|$ and $|E-\nu| \ll\left|\left(g^{2}-\nu / \gamma_{0}\right) \gamma_{1}\right|$, the scattering amplitudes in Eqs. (39) reduce to those in Eqs. (25) with the substitution $g^{2} \rightarrow g^{2}-\nu / \gamma_{0}$. This energy region includes the $D^{* 0} \bar{D}^{0}$ threshold if $\left|\gamma_{1}\right| \gg\left|\gamma_{0} \nu /\left(\nu-g^{2} \gamma_{0}\right)\right|$.

\section{6. $\quad D^{* 0} \bar{D}^{0}$ threshold region}

The interaction parameters $\gamma_{0}, \gamma_{1}, g$, and $\nu$ can be tuned so that there is a bound state just below the $D^{* 0} \bar{D}^{0}$ threshold that can be identified with the $X(3872)$. The scattering amplitudes will have poles at a complex energy $E_{\text {pole }}$ that satisfies Eq. (11) with $\gamma \equiv \kappa\left(E_{\text {pole }}\right)$. The denominator defined in Eq. (40) must vanish at $E_{\text {pole }}$. If the tiny difference between $\kappa_{1}\left(E_{\text {pole }}\right)$ and $\kappa_{11}$ is neglected, the equation $D\left(E_{\text {pole }}\right)=0$ reduces to a cubic polynomial equation for $\gamma$, which can be written

$$
\begin{array}{r}
{\left[\gamma_{1} \kappa_{11}+\left(\gamma_{1}-2 \kappa_{11}\right) \gamma\right]\left[2 \mu\left(\nu-g^{2} \gamma_{0}\right)+\gamma^{2}+i \mu \Gamma_{* 0}\right]} \\
=\gamma_{0}\left(2 \gamma_{1}-\kappa_{11}-\gamma\right)\left(2 \mu \nu+\gamma^{2}+i \mu \Gamma_{* 0}\right)
\end{array}
$$

The general behavior of the three poles has been analyzed in Ref. [27] for the case $\Gamma_{* 0}=0$. The approximate fine tuning required to obtain a small inverse scattering length can be obtained by setting $\gamma=0$ and $\Gamma_{* 0}=0$ :

$$
\gamma_{1} \kappa_{11}\left(\nu-g^{2} \gamma_{0}\right) \approx \gamma_{0}\left(2 \gamma_{1}-\kappa_{11}\right) \nu
$$

If $\left|\gamma_{1}\right| \gg \kappa_{11}$, this fine-tuning condition reduces to $1 / \gamma_{0}-g^{2} / \nu \approx 2 / \kappa_{11}$. An inverse scattering length $\gamma$ that is small compared to $\kappa_{11}$ can be obtained by fine-tuning $\gamma_{0}$ to near $\kappa_{11} / 2 \approx$ $+63 \mathrm{MeV}$ with $|\nu| / g^{2} \gg \kappa_{11}$ or by fine-tuning $\nu$ to near $-g^{2} \kappa_{11} / 2$ with $\left|\gamma_{0}\right| \gg \kappa_{11}$.

Since the line shapes near the $D^{* 0} \bar{D}^{0}$ threshold are extremely sensitive to $\gamma$, it is advantageous to take $\gamma$ to be one of the independent interaction parameters. The equation $D\left(E_{\text {pole }}\right)=0$ can be solved for $\gamma_{0}$ as a function of $\gamma_{1}, g, \nu$, and $\gamma$. If the tiny difference between $\kappa_{1}\left(E_{\text {pole }}\right)$ and $\kappa_{11}$ is neglected, the solution is

$$
\gamma_{0}=\left(\frac{\left(2 \gamma_{1}-\kappa_{11}\right)-\gamma}{\gamma_{1} \kappa_{11}+\left(\gamma_{1}-2 \kappa_{11}\right) \gamma}+\frac{g^{2}}{\nu-E_{\text {pole }}}\right)^{-1}
$$

If the equation for $\gamma$ in Eq. (43) is expanded to first order in the imaginary parts of all the variables, one can solve for the imaginary part of $\gamma$ :

$$
\operatorname{Im} \gamma \approx \frac{1}{2}\left(\frac{\gamma_{1} \kappa_{11}}{\left(\gamma_{1}-\kappa_{11}\right) \gamma_{0}}\right)^{2} \operatorname{Im} \gamma_{0}+\frac{1}{2}\left(\frac{\kappa_{11}}{\gamma_{1}-\kappa_{11}}\right)^{2} \operatorname{Im} \gamma_{1}-\frac{1}{2}\left(\frac{g \gamma_{1} \kappa_{11}}{\left(\gamma_{1}-\kappa_{11}\right) \nu}\right)^{2}\left(\operatorname{Im} \nu+\frac{1}{2} \Gamma_{* 0}\right) .
$$

We have simplified the coefficients by setting $\gamma=0$. We have also used the fine-tuning condition in Eq. (44) to express the coefficients in a manifestly positive form.

The scattering amplitudes $f_{i j}(E)$ in Eqs. (39) have poles at $\kappa(E)=\gamma$ with residues $Z_{i}^{1 / 2} Z_{j}^{1 / 2}$. If the tiny difference between $\kappa_{1}\left(E_{\text {pole }}\right)$ and $\kappa_{11}$ is neglected, the ratio of the residue factors is

$$
\frac{Z_{1}^{1 / 2}}{Z_{0}^{1 / 2}}=-\frac{\gamma_{1}-\gamma}{\gamma_{1}-\kappa_{11}}
$$


This ratio does not depend on the resonance parameters $\nu$ and $g$. The residue $Z_{0}$ is

$$
Z_{0}=\left[1+\frac{\left(\gamma_{1}-\gamma\right)^{2} \gamma}{\left(\gamma_{1}-\kappa_{11}\right)^{2} \kappa_{11}}+\frac{g^{2}\left[\gamma_{1} \kappa_{11}+\left(\gamma_{1}-2 \kappa_{11}\right) \gamma\right]^{2} \gamma}{2 \mu\left(\gamma_{1}-\kappa_{11}\right)^{2}\left(\nu-E_{\text {pole }}\right)^{2}}\right]^{-1}
$$

As $\gamma \rightarrow 0$, this residue approaches 1 . If the small value of $\gamma$ arises from either the fine tuning $\gamma_{0} \approx \kappa_{11} / 2$ or the fine tuning $\nu \approx-g^{2} \kappa_{11} / 2$, the scattering amplitudes $f_{i j}(E)$ in the entire $D^{* 0} \bar{D}^{0}$ threshold region defined by $|E| \ll \nu_{11}=8.1 \mathrm{MeV}$ are dominated by the pole at $\kappa(E)=\gamma$. They reduce to the universal scattering amplitude $f(E)$ given in Eq. (7) multiplied by residue factors:

$$
f_{i j}(E) \approx Z_{i}^{1 / 2} f(E) Z_{j}^{1 / 2}
$$

An inverse scattering length $\gamma$ that is small compared to $\kappa_{11}$ can also be obtained by a double fine-tuning of $\gamma_{0}$ and $\nu$ so that they satisfy $|\nu| \ll g^{2} \kappa_{11},\left|\gamma_{0}\right| \ll \kappa_{11}$, and $\left|\nu-g^{2} \gamma_{0}\right| \ll$ $|\nu|$. In this case, the residue $Z_{0}$ in Eq. (48) can be significantly smaller than 1 . The energy dependence of $f_{i j}(E)$ can also differ significantly from that of the universal amplitude in Eq. (7).

\section{Resonance far above $D^{*} \bar{D}$ threshold}

One region of parameter space in which the amplitudes simplify is when the resonance parameter $\nu$ is much larger than the energy scale $\nu_{11}=8.1 \mathrm{MeV}$ associated with isospin splitting. In this case, the existence of the $X(3872)$ requires the fine-tuning $\gamma_{0} \approx \kappa_{11} / 2$. In addition to the $X(3872)$ resonance just below the $D^{* 0} \bar{D}^{0}$ threshold, there is a second resonance $\chi$ well above the $D^{*+} D^{-}$threshold. This second resonance could be identified with the P-wave charmonium state $\chi_{c 1}^{\prime}$.

We first consider this system at energies in the $D^{*} \bar{D}$ threshold region $|E| \lesssim \nu_{11}$. In this region, the scattering amplitudes in Eqs. (39) reduce to those in Eqs. (17) for the Zero-Range model with the substitution $\gamma_{0} \rightarrow \gamma_{0} \nu /\left(\nu-g^{2} \gamma_{0}\right)$.

We next consider this system at energies in the $\chi$ resonance region. In this region, the difference between $\kappa(E)$ and $\kappa_{1}(E)$ can be neglected and the resonance propagator in Eq. (41) reduces to

$$
P(E) \approx \frac{1}{E-\nu+g^{2} /\left[1 / \gamma_{0}-1 / \kappa(E)\right]} .
$$

It has a pole at a complex energy $E_{\chi}-i \Gamma_{\chi} / 2$ near $\nu-g^{2} \gamma_{0}$. The position of the pole can be calculated by iterating around this approximate solution. The solution to first order in $\gamma_{0} / \kappa\left(E_{\chi}\right)$ is

$$
E_{\chi}-i \Gamma_{\chi} / 2 \approx \nu-g^{2} \gamma_{0}-i \frac{g^{2} \gamma_{0}^{2}}{\sqrt{2 \mu\left(\nu-g^{2} \gamma_{0}\right)}} .
$$

If $\gamma_{0}$ and $\nu$ have small imaginary parts, the energy and width of the resonance are approximately

$$
\begin{aligned}
& E_{\chi} \approx \operatorname{Re}\left(\nu-g^{2} \gamma_{0}\right) \\
& \Gamma_{\chi} \approx-2 \operatorname{Im} \nu+2 g^{2} \operatorname{Im} \gamma_{0}+\frac{2 g^{2}\left(\operatorname{Re} \gamma_{0}\right)^{2}}{\sqrt{2 \mu \operatorname{Re}\left(\nu-g^{2} \gamma_{0}\right)}}
\end{aligned}
$$


The last term in Eq. (52b) is the partial width for decays into $D^{*} \bar{D}$ and $D \bar{D}^{*}$. The terms proportional to $\operatorname{Im} \nu$ and $\operatorname{Im} \gamma_{0}$ correspond to other decay channels of $\chi_{c 1}^{\prime}$ and to inelastic isospin-0 scattering channels for charm meson pairs, respectively. As the energy $E_{\chi}$ is increased by adjusting $\nu$, the partial width into $D^{*} \bar{D}$ and $D \bar{D}^{*}$ decreases to 0 as $E_{\chi}^{-1 / 2}$. This behavior is characteristic of a Feshbach resonance [28].

The scattering amplitudes also simplify near the resonance. Using the approximation $\left|\gamma_{0}\right| \ll\left|2 \mu E_{\chi}\right|^{1 / 2}$, they reduce to

$$
f_{00}(E) \approx f_{11}(E) \approx-f_{01}(E) \approx-\frac{\gamma_{0}^{2}}{2 \mu E_{\chi}} f_{\mathrm{BW}}(E)
$$

where $f_{\mathrm{BW}}(E)$ is the Breit-Wigner amplitude in Eq. (34).

\section{ESTIMATES OF THE INTERACTION PARAMETERS}

The scattering amplitudes for the Zero-Range+Resonance model in Section III D depend on the interaction parameters $\gamma, \gamma_{1}, g$, and $\nu$. In this section, we analyze the constraints on these parameters.

\section{A. Inverse scattering length}

The complex inverse scattering length $\gamma$ can be determined from measurements of the position and width of the $X(3872)$ resonance in the $J / \psi \pi^{+} \pi^{-}$decay channel. The position of the $X(3872)$ resonance relative to the $D^{* 0} \bar{D}^{0}$ threshold is given in Eq. (1). There are only upper limits on the width of the $X(3872)$ resonance in the $J / \psi \pi^{+} \pi^{-}$decay channel. The upper limit from combining the results of the Belle and Babar Collaborations $[1,12]$ is

$$
\Gamma_{X}<2.2 \mathrm{MeV} \quad(90 \% \text { C.L. }) .
$$

A lower bound on the width $\Gamma_{X}$ is the width $\Gamma_{* 0}$ of the constituent $D^{* 0}$, which is given in Eq. (5b). This contribution to the width of the $X(3872)$ can be identified with the decay modes $\left(D^{0} \bar{D}^{0} \pi^{0}, D^{0} \bar{D}^{0} \gamma\right)$.

We denote the real and imaginary parts of the complex inverse scattering length by $\gamma_{\mathrm{re}}$ and $\gamma_{\mathrm{im}}$ :

$$
\gamma=\gamma_{\mathrm{re}}+i \gamma_{\mathrm{im}}
$$

An alternative pair of variables that can in principle be measured directly are the peak position $E_{\max }$ of the resonance and its full width at half-maximum $\Gamma_{\text {fwhm. }}$. The variables $E_{\max }$ and $\Gamma_{\text {fwhm }}$ are functions of $\gamma_{\mathrm{re}}, \gamma_{\mathrm{im}}$, and the $D^{* 0}$ width $\Gamma_{* 0}$. They can be expanded in powers of $\Gamma_{* 0}[22]$ :

$$
\begin{aligned}
E_{\max } & =-\frac{\gamma_{\mathrm{re}}^{2}}{2 \mu}-\frac{\gamma_{\mathrm{im}}}{2 \gamma_{\mathrm{re}}} \Gamma_{* 0}+\ldots, \\
\Gamma_{\mathrm{fwhm}} & =\frac{2 \gamma_{\mathrm{re}} \gamma_{\mathrm{im}}}{\mu}+\Gamma_{* 0}+\ldots
\end{aligned}
$$

In the expression for $\Gamma_{\text {fwhm }}$ in Eq. $(56 \mathrm{~b})$, the second term $\Gamma_{* 0}$ can be identified with the partial width for decay into $\left(D^{0} \bar{D}^{0} \pi^{0}, D^{0} \bar{D}^{0} \gamma\right)$ while the first term can be identified with the partial width into other decay modes. 


\begin{tabular}{l|cc|c} 
Reference & Mass $(\mathrm{MeV})$ & Partial width $(\mathrm{MeV})$ & $g$ \\
\hline BG [32] & 3953 & 118 & 0.37 \\
ELQ [33] & 3968 & 150 & 0.40 \\
BGS [34] & 3925 & 165 & 0.46 \\
ELQ [35] & 3920 & 81 & 0.35
\end{tabular}

TABLE I: Results from coupled-channel potential models for the mass of the $\chi_{c 1}(1 P)$, its partial width into $D^{*} \bar{D}$ and $D \bar{D}^{*}$, and the coupling constant $g$ inferred from Eqs. (32).

The result in Eq. (1) can be interpreted as a measurement of $E_{\max }$. Keeping only the leading term in the expansion for $E_{\max }$ in Eq. (56a), we obtain a determination of $\gamma_{\mathrm{re}}$ :

$$
\gamma_{\mathrm{re}}=28_{-20}^{+12} \mathrm{MeV} \text {. }
$$

The result in Eq. (54) can be interpreted as an upper limit on $\Gamma_{\text {fwhm. }}$. Keeping only the first two terms in the expansion for $\Gamma_{\mathrm{fwhm}}$ in Eq. (56b), we obtain an upper limit on the product of $\gamma_{\mathrm{re}}$ and $\gamma_{\mathrm{im}}$ :

$$
0<\gamma_{\mathrm{re}} \gamma_{\mathrm{im}}<(32 \mathrm{MeV})^{2}
$$

\section{B. Resonance parameters}

The resonance parameters are the energy variable $\nu$ and the coupling constant $g$. If $\nu$ is much larger than the energy scale $\nu_{11}=8.1 \mathrm{MeV}$ of isospin splitting, the ZeroRange+Resonance model predicts a narrow resonance $\chi$ whose energy is well above the $D^{*+} D^{-}$threshold. This resonance could be identified with the P-wave charmonium state $\chi_{c 1}^{\prime} \equiv \chi_{c 1}(2 P)$ or a $1^{++} c \bar{c}$ tetraquark meson or some other $1^{++}$meson. If $\chi$ is identified with the $\chi_{c 1}^{\prime}$, we can take advantage of the well-developed charmonium phenomenology based on quark potential models to constrain the resonance parameters.

Predictions from potential models for the mass of $\chi_{c 1}^{\prime}$ range from about $3920 \mathrm{MeV}$ to about $4010 \mathrm{MeV}$ [29-36]. These predictions are all $50 \mathrm{MeV}$ or more higher than the mass of the $X(3872)$. Since the effects of couplings of charmonium states to pairs of charm mesons are not well understood, we cannot exclude the possibility that they shift the mass of the $\chi_{c 1}^{\prime}$ down into the $D^{*} \bar{D}$ threshold region. We will take the real part of $\nu$ to be an adjustable parameter.

We can use potential models to estimate the coupling constant $g$. Conventional potential models contain no information about charm mesons. Coupled-channel potential models include additional interactions that couple a charm-quark and antiquark to pairs of charm mesons. These models can be used to calculate the partial widths for decays of charmonium states into pairs of charm mesons. The partial width of $\chi_{c 1}^{\prime}$ into $D^{*} \bar{D}$ and $D \bar{D}^{*}$ has been calculated using the ${ }^{3} P_{0}$ model $[32,34]$ and the CCC model $[33,35]$. The results for the mass of the $\chi_{c 1}^{\prime}$ and its partial width into $D^{*} \bar{D}$ and $D \bar{D}^{*}$ are given in Table I. Although these coupled-channel potential models allow scattering of charm mesons, there is no reason to expect the scattering lengths to be much larger than the range of charm meson interactions in the absence of the fine-tuning of a charmonium state to the $D^{* 0} \bar{D}^{0}$ threshold. Thus the appropriate limit of the Zero-Range+Resonance model in Sec. III D is the Flatté limit $\gamma_{0}, \gamma_{1} \rightarrow \infty$, in which the charm mesons scatter only through their coupling to the resonance. The energy $E_{\chi}$ and the width $\Gamma_{\chi}$ of the resonance in this limit are given in Eqs. (32). By 
fitting these expressions for the energy and the width, we obtain the coupling constants listed in Table I. The average value is

$$
g=0.40
$$

and the variations are less than 15\%. We will use the value in Eq. (59) in the numerical analysis in Section VII.

The effects of other decay modes of the $\chi_{c 1}^{\prime}$ besides $D^{*} \bar{D}$ and $D \bar{D}^{*}$ can be taken into account through the imaginary part of the parameter $\nu$. The next most important decay modes are expected to be the radiative transitions $\chi_{c 1}^{\prime} \rightarrow J / \psi \gamma$ and $\chi_{c 1}^{\prime} \rightarrow \psi(2 S) \gamma$. The partial widths scale like the cube of the photon energy, so they depend on the mass of the $\chi_{c 1}^{\prime}$. They have been calculated using quark potential models [32-36]. If the mass of the $\chi_{c 1}^{\prime}$ is close to $3872 \mathrm{MeV}$, the partial widths are roughly $10 \mathrm{keV}$ for $J / \psi \gamma$ and roughly $60 \mathrm{keV}$ for $\psi(2 S) \gamma$. These are small enough that the imaginary part of $\nu$ will not have a dramatic effect on the line shapes near the $D^{* 0} \bar{D}^{0}$ threshold. We will therefore set $\operatorname{Im} \nu=0$.

\section{Charm meson scattering parameters}

The charm meson scattering parameters are $\gamma_{0}$ and $\gamma_{1}$. We first consider the natural scale for these parameters. Since the low-energy scattering of charm mesons is dominated by pion exchange, the obvious estimate for the range of the interaction is $1 / m_{\pi}$. The corresponding estimate of the natural scale for $\gamma_{0}$ and $\gamma_{1}$ is $m_{\pi} \approx 140 \mathrm{MeV}$. Suzuki has suggested that the range of the interaction could be much larger than $1 / m_{\pi}[37]$. The denominator of the propagator for the exchange of a $\pi^{0}$ of momentum $\boldsymbol{q}$ between $D^{* 0}$ and $\bar{D}^{0}$ is $q^{2}+m_{\pi^{0}}^{2}-\left(M_{* 0}-M_{0}\right)^{2}$. There is a near cancellation between the terms $m_{\pi^{0}}^{2}$ and $\left(M_{* 0}-M_{0}\right)^{2}$, because $M_{* 0}-M_{0}$ is larger than $m_{\pi^{0}}$ by only $7.1 \mathrm{MeV}$. If we define an effective mass $m$ by expressing the denominator as $q^{2}+m^{2}$, this effective mass is pure imaginary: $m^{2}=-(44 \mathrm{MeV})^{2}$. One might be tempted to take $|m|$ as the natural scale for $\gamma_{0}$ and $\gamma_{1}$, but this would be incorrect. This can be seen by considering the limit $m_{\pi^{0}} \rightarrow M_{* 0}-M_{0}$, which implies $m \rightarrow 0$. In this limit, the potential from the exchange of $\pi^{0}$ reduces to a $1 / r^{3}$ potential that couples the S-wave and D-wave components of the wavefunction. Although this is a long-range potential, it is not a scale-invariant potential. The coefficient $C_{3}$ in the potential $C_{3} / r^{3}$ provides a scale. In Ref. [38], this coefficient was expressed as $C_{3}=g^{2} /\left(4 \pi f_{\pi}^{2}\right)$, where $g / f_{\pi}$ is the coupling constant for the $D^{*}-D \pi$ interaction. The corresponding momentum scale is $\left(2 \mu C_{3}\right)^{-1}=360 \mathrm{MeV}$. This is the natural scale for $\gamma_{0}$ and $\gamma_{1}$ when $m=0$.

Phenomenological estimates of $\gamma_{0}$ and $\gamma_{1}$ can be obtained from any model that can be used to calculate the scattering amplitudes for charm mesons. One such class of models is meson potential models, in which the degrees of freedom are mesons and their interactions are defined by potentials. The simplest such model is one in which the charm mesons interact only through the exchange of pions [38-40]. More elaborate meson potential models include the effects of the exchange of other mesons [25, 41] or the exchange of quarks [42]. These models typically require an ultraviolet cutoff to regularize singularities in the potentials at short distances. The scattering amplitudes can be calculated by solving the Schrödinger equation. Another class of models that can be used to calculate the scattering amplitudes are meson scattering models, which are defined by scattering parameters. In the simplest such models, such as the universal theory described in Section III A and the Zero-Range model described in Section III B, the only degrees of freedom are charm mesons. Other hadrons 
can also be included as degrees of freedom in meson scattering models. The Flatté model in Section III C and the Zero-Range+Resonance model in Section III D include a resonance that can be identified with the charmonium state $\chi_{c 1}^{\prime}$. More elaborate meson scattering models that include many other mesons as degrees of freedom have also been considered [43]. In meson scattering models, the scattering amplitudes are calculated by solving integral equations, such as the Lippmann-Schwinger equation. These models typically require an ultraviolet cutoff to regularize singularities at large momenta. If the model is renormalizable, like those described in Sections III A, III B, III C, and III D, all dependence on the cutoff can be absorbed into the scattering parameters. Although many meson potential models and meson scattering models have been applied to the $X(3872)$, charm meson scattering lengths have not been calculated in these models. Thus the published results for these models do not provide any direct estimates of the scattering parameters $\gamma_{0}$ and $\gamma_{1}$.

A particularly convenient observable for constraining $\gamma_{0}$ and $\gamma_{1}$ is the ratio of the residues of the poles in the elastic scattering amplitudes $f_{00}(E)$ for $D^{* 0} \bar{D}^{0}$ and $f_{11}(E)$ for $D^{*+} D^{-}$ at the $X(3872)$ resonance. We denote the residue of the pole in $f_{i j}(E)$ at $\kappa(E)=\gamma$ by $Z_{i}^{1 / 2} Z_{j}^{1 / 2}$. The quantity $\left|Z_{1} / Z_{0}\right|^{1 / 2}$ can be interpreted as the ratio of the wavefunctions at the origin for the $\left(D^{*+} D^{-}\right)_{+}$and $\left(D^{* 0} \bar{D}^{0}\right)_{+}$components of the $X(3872)$ [44]. For the Zero-Range+Resonance model, the ratio $Z_{1}^{1 / 2} / Z_{0}^{1 / 2}$ is given in Eq. (47). It is determined primarily by $\gamma_{1}$, because $|\gamma| \ll \kappa_{11}$. It does not depend on the resonance parameters $g$ and $\nu$. This ratio has been calculated in a meson scattering model whose degrees of freedom are $S U(4)$ multiplets of pseudoscalar and vector mesons [43]. The parameters of the model were fine-tuned so that the binding energy of the $X(3872)$ is $0.4 \mathrm{MeV}$. The absolute value of the ratio of the residues was calculated to be $\left|Z_{11} / Z_{00}\right|^{1 / 2}=0.9923$. If we insert the expression for the ratio of the residues in Eq. (47), we obtain two solutions for $\gamma_{1}$ : $+76 \mathrm{MeV}$ and $-12,500 \mathrm{MeV}$. We expect $\gamma_{1}$ to be large compared to $\kappa_{11}=125 \mathrm{MeV}$ and positive, because the pion-exchange interaction is repulsive in the $C=+$ isospin- $1 D^{*} \bar{D}$ channel. Neither of the two solutions for $\gamma_{1}$ are consistent with this expectation. It is possible that the model of [43] does not describe scattering in the isospin-1 channel with sufficient accuracy.

\section{LINE SHAPES OF $X(3872)$ IN $B$ MESON DECAY}

In this section, we consider the line shapes of the $X(3872)$ resonance in the decay $B \rightarrow$ $K+X$. We first present a general formulation of the problem of production by a shortdistance process in terms of an effective field theory that describes the $D^{*} \bar{D}$ threshold region. We summarize the universal line shapes of Ref. [9], which take into account only the large scattering length in the neutral channel. We summarize the line shapes of Ref. [10], which take into account zero-range scattering in the neutral and charged channels. We then describe the Flatté line shapes introduced in Ref. [20], which take into account scattering through a resonance. Finally, we present more general line shapes that allow for both scattering through a resonance and through zero-range interactions. 


\section{A. Effective field theory formulation}

Our starting point for the derivation of the line shapes produced by the decay $B^{+} \rightarrow$ $K^{+}+X$ is the optical theorem for the width of the $B^{+}$:

$$
\Gamma\left[B^{+}\right]=\frac{1}{M_{B}} \operatorname{Im} \mathcal{A}\left[B^{+} \rightarrow B^{+}\right]
$$

where $i \mathcal{A}\left[B^{+} \rightarrow B^{+}\right]$is the one-meson-irreducible forward amplitude for $B^{+}$at leading order in the electroweak interactions and to all orders in QCD interactions. The imaginary part of this amplitude has a contribution from the intermediate state $K^{+}+X(3872)$. In addition to the $X(3872)$ itself, there are other sets of particles with the quantum numbers $J^{P C}=1^{++}$that have enhanced production rates near the $D^{* 0} \bar{D}^{0}$ threshold. We will denote these states collectively by the symbol $\left(1^{++}\right)$. We will use the phrase $X(3872)$ resonance to refer specifically to the peak in the energy distribution just below the $D^{* 0} \bar{D}^{0}$ threshold.

Decays of the $B^{+}$proceed through weak interactions that are mediated by the $W$ boson. Because the mass of the $W$ is so much larger than that of the $B$ meson, the decays can be described completely within QCD using effective field theory methods. The effects of $W$ exchange can be reproduced by an effective weak Hamiltonian. The leading terms that contribute to $B^{+} \rightarrow K^{+}+X$ are current-current operators:

$$
\mathcal{H}_{\text {weak }}=C_{c c} \bar{b} \gamma^{\mu}\left(1-\gamma_{5}\right) c \bar{c} \gamma_{\mu}\left(1-\gamma_{5}\right) s+C_{n c} \bar{b} \gamma^{\mu}\left(1-\gamma_{5}\right) s \bar{c} \gamma_{\mu}\left(1-\gamma_{5}\right) c,
$$

where $C_{c c}$ and $C_{n c}$ are short-distance coefficients. The forward amplitude in Eq. (60) can be expressed as the expectation value in the $B^{+}$meson of the Fourier transform of a bilocal QCD operator:

$$
\mathcal{A}\left[B^{+} \rightarrow B^{+}\right]=i \int d^{4} x\left\langle B^{+}\left|\mathrm{T} \mathcal{H}_{\text {weak }}^{\dagger}(x) \mathcal{H}_{\text {weak }}(0)\right| B^{+}\right\rangle .
$$

The $K^{+}$and $X(3872)$ produced by the decay of $B^{+}$have recoil momenta of $1140 \mathrm{MeV}$, which is large compared to the momentum scale associated with the resonance. If the $X(3872)$ can be described using an effective field theory (EFT) for charm mesons, the decay $B^{+} \rightarrow K^{+}+X(3872)$ can also be described completely within that EFT. In the rest frame of the $X(3872)$, the $B^{+} \rightarrow K^{+}$transition acts like a point source for pairs of charm mesons and possibly other degrees of freedom described by the EFT. As far as the resonance is concerned, the effects of the $B^{+} \rightarrow K^{+}$transition can be reproduced by local EFT operators $\mathcal{O}_{i}^{m}$ acting on the EFT vacuum. The superscript $m$ is a Cartesian vector index and the subscript $i$ labels the various operators. The contribution to the forward amplitude in Eq. (62) from transitions to $K^{+}$that create $1^{++}$states with invariant mass in the $D^{*} \bar{D}$ threshold region can be expressed in terms of expectation values in the EFT vacuum of the Fourier transforms of bilocal EFT operators:

$$
\begin{aligned}
\mathcal{A}_{\left(1^{++}\right)}= & \int \frac{d^{4} P_{K}}{(2 \pi)^{4}} \frac{i}{P_{K}^{2}-m_{K}^{2}+i \varepsilon} \sum_{i, j}\left(C^{j, n}\right)^{*} C^{i, m} \\
& \times i \int d t e^{i E t} \int d^{3} r\left\langle 0\left|\mathrm{TO}_{j}^{n \dagger}(\boldsymbol{r}, t) \mathcal{O}_{i}^{m}(\mathbf{0}, 0)\right| 0\right\rangle .
\end{aligned}
$$

The energy $E$ is defined by $\left(P_{B}-P_{K}\right)^{2}=\left(M_{* 0}+M_{0}+E\right)^{2}$, where $P_{B}$ and $P_{K}$ are the 4momenta of the $B^{+}$and $K^{+}$. The integral over the 4-momentum $P_{K}$ of the $K^{+}$is implicitly 
restricted to the region near the $D^{*} \bar{D}$ threshold where $E$ is small. The short-distance coefficients $C^{i, m}$ specify the linear combinations of EFT operators $\sum_{i} C^{i, m} \mathcal{O}_{i}^{m}$ (with an implied sum on the vector index $m$ ) that reproduce the effects of the $B^{+} \rightarrow K^{+}$transition.

The short-distance coefficients $C^{i, m}$ can in principle be determined by matching matrix elements of $\mathcal{H}_{\text {weak }}$ in QCD with matrix elements of $\sum_{i} C^{i, m} \mathcal{O}_{i}^{m}$ in the EFT. If we consider a $D^{* 0} \bar{D}^{0}$ state in the $D^{*} \bar{D}$ threshold region, the matching condition is

$$
\left\langle K^{+} D^{* 0} \bar{D}^{0}\left|\mathcal{H}_{\text {weak }}(0)\right| B^{+}\right\rangle=\sum_{i} C^{i, m}\left\langle D^{* 0} \bar{D}^{0}\left|\mathcal{O}_{i}^{m}(\mathbf{0}, 0)\right| 0\right\rangle .
$$

Although we cannot calculate the QCD matrix element on the left side, we can use the Lorentz invariance of QCD to determine the tensor structure of the coefficients $C^{i, m}$. We choose the charm meson pair $D^{* 0} \bar{D}^{0}$ to have zero relative momentum. The QCD matrix element on the left side of Eq. (64) must be linear in the polarization 4-vector $\epsilon^{\mu}$ of the $D^{* 0}$ and it can depend only on the external 4-momenta $P_{B}, P_{K}$, and $P_{X} \equiv P_{B}-P_{K}$ of the $B^{+}$, $K^{+}$, and $D^{* 0} \bar{D}^{0}$ pair. Since the operator $\mathcal{H}_{\text {weak }}$ is a Lorentz scalar and $P_{X} \cdot \epsilon=0$, the QCD matrix element must be $P_{B} \cdot \epsilon$ multiplied by a constant. The EFT matrix element on the right side of Eq. (64) must be linear in the polarization 3-vector $\boldsymbol{\epsilon}$ of the $D^{* 0}$ in the $D^{* 0} \bar{D}^{0}$ center-of-mass frame and there are no 3-momenta that it can depend on. Since the operator $\mathcal{O}_{i}^{m}$ is a Cartesian vector with index $m$, the EFT matrix element on the left side of Eq. (64) must be $\epsilon^{m}$ multiplied by a constant. By matching the two sides of Eq. (64), we conclude that the short-distance coefficients must have the form

$$
C^{i, m}=\frac{1}{\sqrt{M_{B}}} C_{B^{+}}^{K^{+}, i} P_{B}^{\mu} L_{\mu}^{m}
$$

where the coefficients $C_{B^{+}}^{K^{+}, i}$ are complex numbers and $L_{\mu}^{m}$ is the boost matrix from a general Lorentz frame to the center-of-mass frame of the $D^{* 0} \bar{D}^{0}$ pair. The prefactor $M_{B}^{-1 / 2}$ has been inserted for later convenience. The boost matrix satisfies $P_{X}^{\mu} L_{\mu}^{m}=0$ and the tensor identities

$$
\begin{aligned}
\delta^{m n} L_{\mu}^{m} L_{\nu}^{n} & =-g_{\mu \nu}+P_{X \mu} P_{X \nu} / P_{X}^{2}, \\
g^{\mu \nu} L_{\mu}^{m} L_{\nu}^{n} & =-\delta^{m n} .
\end{aligned}
$$

The imaginary part of $\mathcal{A}_{\left(1^{++}\right)}$can be obtained from Eq. (63) by using cutting rules. The relevant cuts run through the $K^{+}$propagator and through the EFT Green's function:

$$
\begin{aligned}
\operatorname{Im} \mathcal{A}_{\left(1^{++}\right)}= & \frac{1}{M_{B}} \sum_{i, j} C_{B^{+}}^{K^{+}, i}\left(C_{B^{+}}^{K^{+}, j}\right)^{*} \int \frac{d^{4} P_{K}}{(2 \pi)^{4}} 2 \pi \delta\left(P_{K}^{2}-m_{K}^{2}\right) P_{B}^{\mu} P_{B}^{\nu} L_{\mu}^{m} L_{\nu}^{n} \\
& \times \operatorname{Im} i \int d t e^{i E t} \int d^{3} r\left\langle 0\left|\operatorname{TO}_{j}^{n \dagger}(\boldsymbol{r}, t) \mathcal{O}_{i}^{m}(\mathbf{0}, 0)\right| 0\right\rangle .
\end{aligned}
$$

Rotational invariance implies that the EFT matrix element is proportional to $\delta^{m n}$. Using Eq. (66a), the integrand of the integral over $P_{K}$ in Eq. (67) becomes Lorentz invariant. It can be reduced to an integral over $\left(P_{B}-P_{K}\right)^{2}$ or, equivalently, $E$ :

$$
\int \frac{d^{4} P_{K}}{(2 \pi)^{4}} 2 \pi \delta\left(P_{K}^{2}-m_{K}^{2}\right) P_{B}^{\mu} P_{B}^{\nu}\left(L_{\mu}^{m} L_{\nu}^{n}\right) \delta^{m n}=\int d E \frac{\lambda^{3 / 2}\left(M_{B}, m_{K}, M_{* 0}+M_{0}+E\right)}{32 \pi^{2} M_{B}^{2}\left(M_{* 0}+M_{0}+E\right)} .
$$


In the $D^{*} \bar{D}$ threshold region, the energy $E$ is negligible compared to $M_{* 0}+M_{0}$. The amplitude in Eq. (67) therefore reduces to

$$
\operatorname{Im} \mathcal{A}_{\left(1^{++}\right)}=\frac{\lambda^{3 / 2}\left(M_{B}, m_{K}, M_{* 0}+M_{0}\right)}{96 \pi^{2} M_{B}^{3}\left(M_{* 0}+M_{0}\right)} \sum_{i, j} C_{B^{+}}^{K^{+}, i}\left(C_{B^{+}}^{K^{+}, j}\right)^{*} \int d E \operatorname{Im} F_{i j}(E),
$$

where $F_{i j}(E)$ is the EFT matrix element:

$$
F_{i j}(E)=i \int d t e^{i E t} \int d^{3} r\left\langle 0\left|\mathrm{TO}_{j}^{m \dagger}(\boldsymbol{r}, t) \mathcal{O}_{i}^{m}(\mathbf{0}, 0)\right| 0\right\rangle .
$$

Note that there is an implied sum over the repeated Cartesian vector index $m$. Inserting Eq. (69) into Eq. (60), we get a factorization formula for the inclusive energy distribution summed over $1^{++}$states:

$$
\frac{d \Gamma}{d E}\left[B^{+} \rightarrow K^{+}+\left(1^{++}\right)\right]=\Gamma_{B}^{K} \sum_{i, j} C_{B^{+}}^{K^{+}, i}\left(C_{B^{+}}^{K^{+}, j}\right)^{*} \operatorname{Im} F_{i j}(E) .
$$

The prefactor has dimensions of energy:

$$
\Gamma_{B}^{K}=\frac{\lambda^{3 / 2}\left(M_{B}, m_{K}, M_{* 0}+M_{0}\right)}{96 \pi^{2} M_{B}^{4}\left(M_{* 0}+M_{0}\right)} .
$$

If the short-distance coefficients $C_{B^{+}}^{K^{+}, i}$ are chosen to be dimensionless, the functions $F_{i j}(E)$ have the same dimensions (momentum $)^{-1}$ as a scattering amplitude.

\section{B. Universal line shapes}

In Ref. [9], Braaten and Lu derived expressions for the universal line shapes for $1^{++}$states near the $D^{* 0} \bar{D}^{0}$ threshold produced by the decays $B \rightarrow K+\left(1^{++}\right)$that take into account the large scattering length in the neutral channel $\left(D^{* 0} \bar{D}^{0}\right)_{+}$. The $D^{* 0} \bar{D}^{0}$ threshold region can be described by an effective field theory for the neutral charm mesons $D^{0}, D^{* 0}, \bar{D}^{0}$, and $\bar{D}^{* 0}$ in which the only interaction is zero-range scattering in the $\left(D^{* 0} \bar{D}^{0}\right)_{+}$channel. The scattering amplitude $f(E)$ is given in Eq. (7), with $\kappa(E)$ given by Eq. (10). It depends on a single interaction parameter $\gamma$.

The effects of the $B^{+} \rightarrow K^{+}$transition can be reproduced in this effective field theory by a local operator $\mathcal{O}_{0}^{m}$ with a Cartesian vector index $m$ that creates a pair of charm mesons in the $\left(D^{* 0} \bar{D}^{0}\right)_{+}$channel. The Green's function defined by Eq. (70) for this operator is proportional to the scattering amplitude $f(E)$. The normalization of the operator $\mathcal{O}_{0}^{n}$ can be chosen so that $F_{00}(E)$ is exactly $f(E)$. The resulting factorization formula for the inclusive energy distribution of $1^{++}$states produced by the transition $B^{+} \rightarrow K^{+}$is

$$
\frac{d \Gamma}{d E}\left[B^{+} \rightarrow K^{+}+\left(1^{++}\right)\right]=\Gamma_{B}^{K}\left|C_{B^{+}}^{K^{+}, 0}\right|^{2} \operatorname{Im} f(E),
$$

where $C_{B^{+}}^{K^{+}, 0}$ is a complex short-distance coefficient. The imaginary part of the scattering amplitude in Eq. (73) is given by the optical theorem in Eq. (8). The term proportional to $\operatorname{Im} \kappa(E)$ is the contribution from channels whose ultimate final states are $\left(D^{0} \bar{D}^{0} \pi^{0}, D^{0} \bar{D}^{0} \gamma\right)$. The term proportional to $\operatorname{Im} \gamma$ is the contribution from all other channels. 
The inclusive energy distribution for $1^{++}$states produced by the transition $B^{0} \rightarrow K^{0}$ is given by an expression identical to Eq. (73) except that $C_{B^{+}}^{K^{+}, 0}$ is replaced by an independent short-distance coefficient $C_{B^{0}}^{K^{0}, 0}$. Since the rate in Eq. (73) does not depend on the phase of $C_{B^{+}}^{K^{+}, 0}$, the short-distance coefficients for the $B^{+} \rightarrow K^{+}$and $B^{0} \rightarrow K^{0}$ transitions depend on only two independent real constants.

\section{Zero-range line shapes}

In Ref. [10], the universal line shapes in Section V B were generalized to the Zero-Range model of Section III B. This model can be derived from a renormalizable quantum field theory for neutral and charged charm mesons with zero-range interactions among the channels $\left(D^{* 0} \bar{D}^{0}\right)_{+}$and $\left(D^{*+} D^{-}\right)_{+}$. The effects of the $B^{+} \rightarrow K^{+}$transition can be reproduced by a linear combination of two local operators that create pairs of charm mesons in the $\left(D^{* 0} \bar{D}^{0}\right)_{+}$ and $\left(D^{*+} D^{-}\right)_{+}$channels. The two operators mix under renormalization. For an appropriate choice of the renormalized operators $\mathcal{O}_{0}^{n}$ and $\mathcal{O}_{1}^{n}$, the Green's functions $F_{i j}(E)$ defined by Eq. (70) are simply the scattering amplitudes $f_{i j}(E)$. The resulting factorization formula for the inclusive energy distribution of $1^{++}$states produced by the transition $B^{+} \rightarrow K^{+}$is

$$
\frac{d \Gamma}{d E}\left[B^{+} \rightarrow K^{+}+\left(1^{++}\right)\right]=\Gamma_{B}^{K} \sum_{i, j} C_{B^{+}}^{K^{+}, i}\left(C_{B^{+}}^{K^{+}, j}\right)^{*} \operatorname{Im} f_{i j}(E),
$$

where the sums are over $i, j \in\{0,1\}$. The short-distance coefficients $C_{B^{+}}^{K^{+}, i}$ can be complex. Since the rate in Eq. (74) does not depend on a common phase in $C_{B^{+}, 0}^{K^{+},}$and $C_{B^{+}}^{K^{+}, 1}$, the shortdistance coefficients are determined by three independent real parameters. The scattering amplitudes $f_{i j}(E)$ are given in Eqs. (17) and expressions for their imaginary parts are given in Eq. (20). Note that Eq. (20) gives two different decompositions of the imaginary part of the function $f_{10}(E)=f_{01}(E)$ into terms linear in the imaginary parts of $\kappa(E), \kappa_{1}(E), \gamma_{0}$, and $\gamma_{1}$. To obtain an expression for $d \Gamma / d E$ that is consistent with the Cutkosky cutting rules, these two different expressions must be inserted for $\operatorname{Im} f_{01}(E)$ and $\operatorname{Im} f_{10}(E)$ in Eq. (74).

The inclusive energy distribution for $1^{++}$states produced by the transition $B^{0} \rightarrow K^{0}$ is given by an expression identical to Eq. (74) except that the short-distance coefficients $C_{B^{+}}^{K^{+}, i}$ are replaced by $C_{B^{0}}^{K^{0}, i}$. Since the effective weak hamiltonian $\mathcal{H}_{\text {weak }}$ in Eq. (61) is an isospin scalar, the operator $\sum_{i} C^{i, m} \mathcal{O}_{i}^{m}$ must also be an isospin scalar. The approximate isospin symmetry of QCD can then be used to relate $C_{B^{0}}^{K^{0}, i}$ to $C_{B^{+}}^{K^{+}, i}[10]$ :

$$
\begin{aligned}
& C_{B^{0}}^{K^{0}, 0}=C_{B^{+}, 1}^{K^{+},}, \\
& C_{B^{0}}^{K^{0}, 1}=C_{B^{+}}^{K^{+}, 0} .
\end{aligned}
$$

Thus the two short-distance coefficients for the $B^{0} \rightarrow K^{0}$ transition are determined by the same three independent real constants as the short-distance coefficients for the $B^{+} \rightarrow K^{+}$ transition. In Ref. [10], the expressions for $C_{B^{0}}^{K^{0}, i}$ had the opposite signs from those in Eqs. (75). This error did not affect any of the physical results in Ref. [10]. 


\section{Flatté line shapes}

In Ref. [20], Hanhart, Nefediev, and Kalashnikova proposed line shapes for the $X(3872)$ resonance in the $D^{* 0} \bar{D}^{0}$ threshold region that correspond to the Flatté parameterization of the $D^{* 0} \bar{D}^{0}$ elastic scattering amplitude in Eq. (26). Their line shapes in the $D^{* 0} \bar{D}^{0}$ and $J / \psi \pi^{+} \pi^{-}$decay channels are

$$
\begin{aligned}
\frac{d \Gamma}{d E}\left[B^{+} \rightarrow K^{+}\left(D^{* 0} \bar{D}^{0}, D^{0} \bar{D}^{* 0}\right)\right] & =\frac{2 \mathcal{B} \Gamma\left[B^{+}\right]}{\pi g^{2}}\left|f_{\text {Flatté }}(E)\right|^{2} \sqrt{2 \mu E} \theta(E), \\
\frac{d \Gamma}{d E}\left[B^{+} \rightarrow K^{+} J / \psi \pi^{+} \pi^{-}\right] & =\frac{2 \mathcal{B} \Gamma\left[B^{+}\right]}{\pi g^{4}}\left|f_{\text {Flatté }}(E)\right|^{2} \Gamma_{J / \psi \pi \pi}(E)
\end{aligned}
$$

where $\Gamma\left[B^{+}\right]$is the total width of the $B^{+}$and $\mathcal{B}$ is a short-distance coefficient associated with the $B^{+} \rightarrow K^{+}$transition. The energy dependence of the function $\Gamma_{J / \psi \pi \pi}(E)$ is the decay rate of a resonance of energy $E$ into $J / \psi \pi^{+} \pi^{-}$through the decay into $J / \psi \rho$ followed by $\rho \rightarrow \pi^{+} \pi^{-}$.

The Flatté line shapes can be derived in the effective field theory framework of Section V A by assuming that the short-distance process is the creation of the resonance through an operator $\mathcal{O}_{2}^{m}$. The Green's function $F_{22}(E)$ is proportional to the propagator of the resonance. The constant factor can be chosen so that $F_{22}(E)$ is equal to the Flatté scattering amplitude $f_{\text {Flatté }}(E)$ in Eq. (26). The inclusive energy distribution in Eq. (71) is then

$$
\frac{d \Gamma}{d E}\left[B^{+} \rightarrow K^{+}+\left(1^{++}\right)\right]=\Gamma_{B}^{K}\left|C_{B^{+}}^{K^{+}, 2}\right|^{2} \operatorname{Im} f_{\text {Flatté }}(E)
$$

where $C_{B^{+}}^{K^{+}, 2}$ is the short-distance coefficient for the operator that creates the resonance. The imaginary part of the Flatté scattering amplitude is given in Eq. (27).

The line shapes in Eqs. (76a) and $(76 \mathrm{~b})$ can be obtained by replacing $\Gamma_{B}^{K}\left|C_{B^{+}}^{K^{+}, 2}\right|^{2}$ by $2 \mathcal{B} \Gamma\left[B^{+}\right] /\left(\pi g^{2}\right)$ and by making the substitutions

$$
\begin{aligned}
-\operatorname{Im} \kappa(E) & \longrightarrow \sqrt{2 \mu E} \theta(E), \\
-\operatorname{Im} \nu & \longrightarrow \Gamma_{J / \psi \pi \pi}(E) / 2 .
\end{aligned}
$$

The line shape for $D^{* 0} \bar{D}^{0}$ in Eq. (76a) vanishes for $E<0$. Thus the substitution in Eq. (78a) discards all contributions to $\left(D^{0} \bar{D}^{0} \pi^{0}, D^{0} \bar{D}^{0} \gamma\right)$ final states from the $X(3872)$ resonance below the $D^{* 0} \bar{D}^{0}$ threshold.

\section{E. Zero-range+resonance line shapes}

We now generalize the line shapes of Sections V C and V D to the Zero-Range+Resonance model of Section IIID, in which the coupled channels $\left(D^{* 0} \bar{D}^{0}\right)_{+}$and $\left(D^{*+} D^{-}\right)_{+}$scatter through a resonance as well as through zero-range interactions.

\section{General case}

In the Zero-Range+Resonance model, the scattering amplitudes $f_{i j}(E)$ are given by Eq. (35) and the resonance propagator $P(E)$ is given in Eq. (36). They depend on the 
three independent entries of the symmetric matrix $\Lambda$, the two components of the column vector $G$, and $\nu$. As described in the Appendix, this model can be derived from a renormalizable field theory for the neutral and charged charm mesons and the resonance $\chi$.

The effects of the $B^{+} \rightarrow K^{+}$transition can be reproduced in this model by a linear combination of three local operators: two of them create pairs of charm mesons in the $\left(D^{* 0} \bar{D}^{0}\right)_{+}$and $\left(D^{*+} D^{-}\right)_{+}$channels and the third creates the resonance. The solution to the renormalization problem for these operators is presented in the Appendix. Two of the renormalized operators $\mathcal{O}_{0}^{n}$ and $\mathcal{O}_{1}^{n}$ can be chosen so that the Green's functions $F_{i j}(E)$ defined in Eq. (70) for $i, j=0,1$ are just the scattering amplitudes:

$$
F_{i j}(E)=f_{i j}(E), \quad i, j \in\{0,1\} .
$$

The remaining operator $\mathcal{O}_{2}^{n}$ can be chosen so that $F_{22}(E)$ is the resonance propagator $P(E)$ given in Eq. (36) multiplied by a constant:

$$
F_{22}(E)=-\left(G^{T} G / 2\right) P(E) .
$$

The multiplicative factor of $G^{T} G$ has been inserted only to ensure that $F_{22}(E)$ has the same dimensions as a scattering amplitude. The two remaining Green's functions $F_{02}(E)$ and $F_{12}(E)$ are then given by

$$
F_{i 2}(E)=\left(G^{T} G / 2\right)^{1 / 2}\left(f(E)\left[(E-\nu) \Lambda+G G^{T}\right]^{-1} G\right)_{i} .
$$

The inclusive energy distribution for $1^{++}$states produced by the transition $B^{+} \rightarrow K^{+}$is given by the factorization formula in Eq. (71), where the sums are over $i, j \in\{0,1,2\}$. The

short-distance coefficients $C_{B^{+}}^{K^{+}, i}$ can be complex. Since the rate in Eq. (71) is not affected by a common phase in $C_{B^{+}}^{K^{+}, i}$, these three coefficients are determined by 5 independent real constants.

\section{Isospin symmetry}

Using the approximate isospin symmetry of QCD, the matrix $\Lambda$ can be expressed in terms of two independent parameters $\gamma_{0}$ and $\gamma_{1}$ using Eq. (15) and the column vector $G$ can be expressed in terms of a single coupling constant $g$ using Eq. (37). The scattering amplitudes $f_{i j}(E)$ are given in Eqs. (39). The remaining Green functions $F_{i j}(E)$ in Eqs. (81) and $(80)$ reduce to

$$
\begin{aligned}
& F_{02}(E)=\frac{g^{2} \gamma_{0}\left[-\gamma_{1}+\kappa_{1}(E)\right]}{D(E)} \\
& F_{12}(E)=-\frac{g^{2} \gamma_{0}\left[-\gamma_{1}+\kappa(E)\right]}{D(E)} \\
& F_{22}(E)=\frac{-g^{2} D_{0}(E)}{2 D(E)}
\end{aligned}
$$

where the denominator $D(E)$ is given in Eq. (40) and $D_{0}(E)$ is given in Eq. (18).

The inclusive energy distribution for $1^{++}$states produced by the transition $B^{0} \rightarrow K^{0}$ is given by a factorization formula identical to Eq. (71) except that the short-distance coefficients $C_{B^{+}}^{K^{+}, i}$ are replaced by $C_{B^{0}}^{K^{0}, i}$. Isospin symmetry implies the relations between $C_{B^{0}}^{K^{0}, i}$ 
and $C_{B^{+}}^{K^{+} i}$ for $i=0,1$ in Eqs. (75). The assumption that the resonance has isospin 0 implies a similar relation between the short-distance coefficients for $i=2$ :

$$
C_{B^{+}}^{K^{+}, 2}=C_{B^{0}}^{K^{0}, 2}
$$

Thus the three complex short-distance coefficients for the $B^{0} \rightarrow K^{0}$ transition are determined by the same five independent real constants as the short-distance coefficients for the $B^{+} \rightarrow K^{+}$transition.

\section{Optical theorem}

The inclusive energy distribution in Eq. (71) depends on the imaginary parts of the amplitudes $F_{i j}(E)$. Expressions for the imaginary parts of $F_{i j}(E)$ for $i, j \in\{0,1\}$ that are consistent with the Cutkosky cutting rules are given in Eqs. (42). We also need expressions for the imaginary parts of $F_{02}(E), F_{12}(E)$, and $F_{22}(E)$ that are consistent with the Cutkosky cutting rules. The imaginary parts of $F_{i 2}(E)=F_{2 i}(E)$ can be expressed as

$$
\begin{aligned}
\operatorname{Im} F_{i 2}(E)= & \frac{g^{2}}{2} \operatorname{Im}\left[f_{i 0}(E)-f_{i 1}(E)\right]\left(\frac{\gamma_{0}}{E-\nu+g^{2} \gamma_{0}}\right)^{*} \\
& +\frac{g^{2}}{2}\left[f_{i 0}(E)-f_{i 1}(E)\right]\left(\frac{E-\nu}{\left|E-\nu+g^{2} \gamma_{0}\right|^{2}} \operatorname{Im} \gamma_{0}+\frac{\gamma_{0}}{\left|E-\nu+g^{2} \gamma_{0}\right|^{2}} \operatorname{Im} \nu\right) \\
\operatorname{Im} F_{2 i}(E)= & \frac{g^{2}}{2}\left(\frac{\gamma_{0}}{E-\nu+g^{2} \gamma_{0}}\right) \operatorname{Im}\left[f_{0 i}(E)-f_{1 i}(E)\right] \\
& +\frac{g^{2}}{2}\left(\frac{E-\nu}{\left|E-\nu+g^{2} \gamma_{0}\right|^{2}} \operatorname{Im} \gamma_{0}+\frac{\gamma_{0}}{\left|E-\nu+g^{2} \gamma_{0}\right|^{2}} \operatorname{Im} \nu\right)\left[f_{0 i}^{*}(E)-f_{1 i}^{*}(E)\right]
\end{aligned}
$$

The imaginary parts of $f_{i 0}(E), f_{i 1}(E), f_{0 i}(E)$, and $f_{1 i}(E)$ can be decomposed into terms linear in the imaginary parts of $\kappa(E), \kappa_{1}(E), \gamma_{0}, \gamma_{1}$, and $\nu$ by using Eqs. (42). Note that Eqs. (84a) and (84b) give two different decompositions of the imaginary parts of the function $F_{i 2}(E)=F_{2 i}(E)$. To obtain an expression for $d \Gamma / d E$ that is consistent with the Cutkosky cutting rules, these two different expressions must be inserted for $\operatorname{Im} F_{i 2}(E)$ and $\operatorname{Im} F_{2 i}(E)$ in Eq. (71). The imaginary part of $F_{22}(E)$ can be expressed as

$$
\begin{aligned}
\operatorname{Im} F_{22}(E)= & 2\left|F_{22}\right|^{2}\left[-\frac{1}{g^{2}} \operatorname{Im} \nu\right. \\
& +\left|\frac{-\gamma_{1}\left[\kappa(E)+\kappa_{1}(E)\right]+2 \kappa_{1}(E) \kappa(E)}{D_{0}(E)}\right|^{2} \operatorname{Im} \gamma_{0}+\left|\frac{\gamma_{0}\left[\kappa(E)-\kappa_{1}(E)\right]}{D_{0}(E)}\right|^{2} \operatorname{Im} \gamma_{1} \\
& \left.-2\left|\frac{\gamma_{0}\left[-\gamma_{1}+\kappa_{1}(E)\right]}{D_{0}(E)}\right|^{2} \operatorname{Im} \kappa(E)-2\left|\frac{\gamma_{0}\left[-\gamma_{1}+\kappa(E)\right]}{D_{0}(E)}\right|^{2} \operatorname{Im} \kappa_{1}(E)\right]
\end{aligned}
$$

where $D_{0}(E)$ is the denominator given in Eq. (18). 


\section{4. $\quad D^{* 0} \bar{D}^{0}$ threshold region}

The interaction parameters $\gamma_{0}, \gamma_{1}, \nu$, and $g$ can be tuned so that there is a bound state just below the $D^{* 0} \bar{D}^{0}$ threshold that can be identified with the $X(3872)$. The amplitudes $F_{i j}(E)$ have poles at $\kappa(E)=\gamma$ with residues $Z_{i}^{1 / 2} Z_{j}^{1 / 2}$. The residue factors $Z_{0}^{1 / 2}$ and $Z_{1}^{1 / 2}$ are given by Eqs. (47) and (48). The ratio of $Z_{2}^{1 / 2}$ and $Z_{0}^{1 / 2}$ is

$$
\frac{Z_{2}^{1 / 2}}{Z_{0}^{1 / 2}}=-\frac{g^{2}\left[\gamma_{1} \kappa_{11}+\left(\gamma_{1}-2 \kappa_{11}\right) \gamma\right]}{2\left(\gamma_{1}-\kappa_{11}\right)\left(\nu-E_{\text {pole }}\right)}
$$

If the small value of $\gamma$ is obtained by a single fine-tuning of $\gamma_{0}$ or $\nu$, the behavior of these amplitudes in the entire $D^{* 0} \bar{D}^{0}$ threshold region $|E| \ll \nu_{11} \approx 8.1 \mathrm{MeV}$ is dominated by the pole at $\kappa(E)=\gamma$. They all reduce to the universal scattering amplitude $f(E)$ in Eq. (7) multiplied by residue factors:

$$
F_{i j}(E) \approx Z_{i}^{1 / 2} f(E) Z_{j}^{1 / 2}
$$

The inclusive energy distribution in Eq. (71) simplifies in the $D^{* 0} \bar{D}^{0}$ threshold region:

$$
\frac{d \Gamma}{d E}\left[B^{+} \rightarrow K^{+}+\left(1^{++}\right)\right] \approx \Gamma_{B}^{K}\left|C_{B^{+}}^{K^{+}, 0}-\frac{\gamma_{1}}{\gamma_{1}-\kappa_{11}} C_{B^{+}}^{K^{+}, 1}-\frac{g^{2} \gamma_{1} \kappa_{11}}{2\left(\gamma_{1}-\kappa_{11}\right) \nu} C_{B^{+}}^{K^{+}, 2}\right|^{2} \operatorname{Im} f(E)
$$

where $\operatorname{Im} f(E)$ is given in Eq. (8). The coefficients $Z_{j}^{1 / 2}$ of $C_{B^{+}}^{K^{+}, j}$ have been simplified by taking the limit $\gamma \rightarrow 0$ and by neglecting the imaginary parts of the interaction parameters. The result in Eq. (88) agrees with the universal factorization formula in Eq. (73) after a redefinition of the short-distance coefficient $C_{B^{+}}^{K^{+}, 0}$. By using the expression for $\operatorname{Im} \gamma$ in Eq. (46), the energy-dependent factor in Eq. (88) can be expressed as a linear combination of the imaginary parts of $\gamma_{0}, \gamma_{1}, \nu$, and $\kappa(E)$. There are additional contributions to the coefficients of the imaginary parts of $\gamma_{0}, \gamma_{1}$, and $\nu$ that come from cuts through the factors of $Z_{i}^{1 / 2}$ and $Z_{j}^{1 / 2}$ in Eq. (87), but they are suppressed compared to those in Eq. (88) by factors that are at least as small as $\gamma / \kappa_{11}$ or $\kappa(E) / \kappa_{11}$.

\section{Resonance far above $D^{*} \bar{D}$ threshold}

In the region of parameter space in which $\nu$ is much larger than the energy scale $\nu_{11}=$ 8.1 MeV of isospin splitting, there is a second resonance $\chi$ well above the $D^{*+} D^{-}$threshold in addition to the $X(3872)$ resonance just below the $D^{* 0} \bar{D}^{0}$ threshold. In this case, the amplitudes $F_{i j}(E)$ can be simplified.

For energies $E$ in the $\chi$ resonance region, the line shapes are dominated by a Breit-Wigner resonance whose energy and width are given by Eqs. (52). The scattering amplitudes $f_{i j}(E)$ reduce near the resonance to the expressions in Eq. (53). The remaining amplitudes reduce to

$$
\begin{aligned}
F_{02}(E) \approx-F_{12}(E) & \approx-\frac{i \gamma_{0}}{\sqrt{2 \mu E_{\chi}}} f_{\mathrm{BW}}(E) \\
F_{22}(E) & \approx f_{\mathrm{BW}}(E)
\end{aligned}
$$


where $f_{\mathrm{BW}}(E)$ is the Breit-Wigner scattering amplitude in Eq. (34). Note that $F_{i j}(E)$ differs from $f_{\mathrm{BW}}(E)$ by a factor of $-i \gamma_{0} / \sqrt{2 \mu E_{\chi}}$ for every subscript 0 and by a factor of $+i \gamma_{0} / \sqrt{2 \mu E_{\chi}}$ for every subscript 1 . Thus the expression in Eq. (71) for the inclusive energy distribution reduces near the resonance to

$$
\frac{d \Gamma}{d E}\left[B^{+} \rightarrow K^{+}+\left(1^{++}\right)\right] \approx \Gamma_{B}^{K}\left|-\frac{i \gamma_{0}}{\sqrt{2 \mu E_{\chi}}}\left(C_{B^{+}}^{K^{+}, 0}-C_{B^{+}}^{K^{+}, 1}\right)+C_{B^{+}}^{K^{+}, 2}\right|^{2} \frac{g^{2} \Gamma_{\chi} / 4}{\left(E-E_{\chi}\right)^{2}+\Gamma_{\chi}^{2} / 4} .
$$

Upon integrating over the energy, the partial width from the Breit-Wigner resonance is

$$
\Gamma\left[B^{+} \rightarrow K^{+}+\chi_{c 1}^{\prime}\right] \approx \frac{\pi g^{2}}{2} \Gamma_{B}^{K}\left|-\frac{i \gamma_{0}}{\sqrt{2 \mu E_{\chi}}}\left(C_{B^{+}}^{K^{+}, 0}-C_{B^{+}}^{K^{+}, 1}\right)+C_{B^{+}}^{K^{+}, 2}\right|^{2} .
$$

\section{Zero-range limit}

If the parameters satisfy $|\nu| \gg g^{2}\left|\gamma_{0}\right|$, the Zero-Range+Resonance model reduces to the Zero-Range model in the energy region $|E| \ll|\nu|$. The scattering amplitudes reduce to those in Eqs. (17). The amplitudes $F_{02}(E), F_{12}(E)$, and $F_{22}(E)$ reduce to

$$
\begin{aligned}
& F_{02}(E) \approx-\frac{g^{2} \gamma_{0}\left[-\gamma_{1}+\kappa_{1}(E)\right]}{\nu D_{0}(E)} \\
& F_{12}(E) \approx \frac{g^{2} \gamma_{0}\left[-\gamma_{1}+\kappa(E)\right]}{\nu D_{0}(E)}, \\
& F_{22}(E) \approx \frac{g^{2}}{2 \nu}
\end{aligned}
$$

They are suppressed by a factor of $g^{2} \gamma_{0} / \nu$. Thus the line shapes reduce to those of the Zero-Range model in Eq. (74).

\section{Flatté limit}

If the parameters satisfy $\left|\gamma_{1}\right| \gg \kappa_{11}$ and $\nu \ll g^{2}\left|\gamma_{0}\right|, g^{2}\left|\gamma_{1}\right|$, the Zero-Range+Resonance model reduces to the Flatté model in the energy region $|E| \ll\left|\gamma_{1}\right|^{2} / \mu, g^{2}\left|\gamma_{0}\right|, g^{2}\left|\gamma_{1}\right|$. The scattering amplitudes $f_{i j}(E)$ reduce to those of the Flatté model in Eqs. (25). The remaining amplitudes $F_{02}(E), F_{12}(E)$, and $F_{22}(E)$ reduce to

$$
F_{02}(E) \approx-F_{12}(E) \approx F_{22}(E) \approx f_{\text {Flatté }}(E) .
$$

where $f_{\text {Flatté }}(E)$ is the Flatté scattering amplitude in Eq. (26). Note that $F_{i j}(E)$ differs from $f_{\text {Flatté }}(E)$ by a factor of -1 for every subscript 1 . Thus the expression in Eq. (71) for the inclusive energy distribution reduces to

$$
\frac{d \Gamma}{d E}\left[B^{+} \rightarrow K^{+}+\left(1^{++}\right)\right] \approx \Gamma_{B}^{K}\left|C_{B^{+}}^{K^{+}, 0}-C_{B^{+}}^{K^{+}, 1}+C_{B^{+}}^{K^{+}, 2}\right|^{2} \operatorname{Im} f_{\text {Flatté }}(E)
$$


The imaginary part of the Flatté scattering amplitude is given in Eq. (27). The inclusive energy distribution in Eq. (94) agrees with that for the Flatté model in Eq. (74) after a redefinition of the short-distance coefficient $C_{B^{+}, 2}^{K^{+}}$.

If there is a resonance well above the $D^{*+} D^{-}$threshold that can be identified with $\chi_{c 1}^{\prime}$, the Flatté scattering amplitude $f_{\text {Flatté }}(E)$ in Eq. (26) reduces near the resonance to the Breit-Wigner amplitude in Eq. (34). Upon integrating over the energy, the partial width from the Breit-Wigner resonance is

$$
\Gamma\left[B^{+} \rightarrow K^{+}+\chi_{c 1}^{\prime}\right] \approx \frac{\pi g^{2}}{2} \Gamma_{B}^{K}\left|C_{B^{+}}^{K^{+}, 0}-C_{B^{+}}^{K^{+}, 1}+C_{B^{+}}^{K^{+}, 2}\right|^{2} .
$$

\section{CONSTRAINTS ON THE $B \rightarrow K$ TRANSITION COEFFICIENTS}

In this section, we analyze the constraints on the short-distance coefficients for the $B \rightarrow K$ transitions from information about $B$ meson decays.

\section{A. $B$ meson decays into $K+X(3872)$}

Products of the branching fractions for the decay $B \rightarrow K+X(3872)$ followed by the decay of $X(3872)$ into specific final states have been measured by the Belle and Babar Collaborations. The most precise measurements are for the final state $J / \psi \pi^{+} \pi^{-}[12,13]$ and for the final states $D^{0} \bar{D}^{0} \pi^{0}[7]$ and $D^{0} \bar{D}^{0} \gamma[15,16]$. We denote the final states $D^{0} \bar{D}^{0} \pi^{0}$ and $D^{0} \bar{D}^{0} \gamma$ collectively by $\left(D^{0} \bar{D}^{0} \pi^{0}, D^{0} \bar{D}^{0} \gamma\right)$. The measurements for the final state $D^{0} \bar{D}^{0} \pi^{0}$ in Ref. [7] can be interpreted as measurements for $\left(D^{0} \bar{D}^{0} \pi^{0}, D^{0} \bar{D}^{0} \gamma\right)$ multiplied by a $62 \%$ branching fraction. Since $D^{* 0}$ ultimately decays into $D^{0} \pi^{0}$ or $D^{0} \gamma$, the measurements for the final states $D^{* 0} \bar{D}^{0}$ and $D^{0} \bar{D}^{* 0}$ in Refs. $[15,16]$ can be interpreted as measurements for $\left(D^{0} \bar{D}^{0} \pi^{0}, D^{0} \bar{D}^{0} \gamma\right)$. The measurements for $B^{+}$decays are more precise than those for $B^{0}$ decays. We introduce a simple notation for the product of branching fractions in $B^{+}$decays:

$$
\mathrm{Br}_{+}[\text {final }] \equiv \operatorname{Br}\left[B^{+} \rightarrow K^{+}+X(3872) \rightarrow K^{+}+\text {final }\right]
$$

Another convenient observable is the ratio $R_{0+}$ of the products of branching fractions for $B^{0}$ decays and $B^{+}$decays:

$$
R_{0+}[\text { final }] \equiv \frac{\operatorname{Br}\left[B^{0} \rightarrow K^{0}+X(3872) \rightarrow K^{0}+\text { final }\right]}{\operatorname{Br}\left[B^{+} \rightarrow K^{+}+X(3872) \rightarrow K^{+}+\text {final }\right]}
$$

The measurements of $\mathrm{Br}_{+}$and $R_{0+}$ are summarized in Table II. To obtain the average

values of the measurements for $J / \psi \pi^{+} \pi^{-}$and $\left(D^{0} \bar{D}^{0} \pi^{0}, D^{0} \bar{D}^{0} \gamma\right)$ in Table II, statistical and systematic errors were added in quadrature. The ratio of $R_{0+}$ for $\left(D^{0} \bar{D}^{0} \pi, D^{0} \bar{D}^{0} \gamma\right)$ and $R_{0+}$ for $J / \psi \pi^{+} \pi^{-}$is $2.1 \pm 0.9$, which differs from 1 by more than 1 standard deviation. The ratio of $\mathrm{Br}_{+}$for $\left(D^{0} \bar{D}^{0} \pi, D^{0} \bar{D}^{0} \gamma\right)$ and $\mathrm{Br}_{+}$for $J / \psi \pi^{+} \pi^{-}$is $11.0 \pm 2.5$. For reasons that will be discussed below, this result does not necessarily imply that the branching fraction for $X(3872)$ into $\left(D^{0} \bar{D}^{0} \pi, D^{0} \bar{D}^{0} \gamma\right)$ is an order of magnitude larger than for $J / \psi \pi^{+} \pi^{-}$.

In the Zero-Range+Resonance model, the products of branching fractions can be calculated in terms of the scattering parameters $\gamma, \gamma_{1}, g$, and $\nu$ and the $B \rightarrow K$ transition coefficients $C_{B^{+}}^{K^{+}, i}, i=1,2,3$. The inclusive energy distribution for $1^{++}$states is given in 


\begin{tabular}{l|cll} 
Reference & Decay mode & $\mathrm{Br}_{+} \times 10^{6}$ & $R_{0+}$ \\
\hline Babar [12] & $J / \psi \pi^{+} \pi^{-}$ & $8.4 \pm 1.5 \pm 0.7$ & $0.41 \pm 0.24 \pm 0.05$ \\
Belle [13] & $J / \psi \pi^{+} \pi^{-}$ & $8.10 \pm 0.92 \pm 0.66$ & $0.82 \pm 0.22 \pm 0.05$ \\
Average & $J / \psi \pi^{+} \pi^{-}$ & $8.2 \pm 0.9$ & $0.63 \pm 0.17$ \\
\hline Belle [7] & $D^{0} \bar{D}^{0} \pi^{0}$ & $102 \pm 31_{-29}^{+21}$ & $1.63 \pm 1.03$ \\
Babar [15] & $D^{* 0} \bar{D}^{0}, D^{0} \bar{D}^{* 0}$ & $167 \pm 36 \pm 47$ & $1.33 \pm 0.69 \pm 0.43$ \\
Belle [16] & $D^{* 0} \bar{D}^{0}, D^{0} \bar{D}^{* 0}$ & $77 \pm 16 \pm 10$ & $1.26 \pm 0.65 \pm 0.06$ \\
Average & $\left(D^{0} \bar{D}^{0} \pi^{0}, D^{0} \bar{D}^{0} \gamma\right) 90 \pm 17$ & $1.35 \pm 0.46$
\end{tabular}

TABLE II: Data on products of branching fractions for $B \rightarrow K+X(3872)$ followed by the decay of $X$ into specified decay modes. The first column gives the reference for the experimental result by the Belle or Babar Collaboration. The second column specifies the decay mode for $X(3872)$. The third column gives the product $\mathrm{Br}_{+}$of the branching fractions for $B^{+}$decays. The fourth column gives the ratio $R_{0+}$ of the products of the branching fractions for $B^{0}$ decays and $B^{+}$decays. Results with both a statistical and a systematic error are experimental results. Results with a single error were obtained by combining statistical and systematic errors in quadrature.

Eq. (71). The imaginary parts of the amplitudes $F_{i j}(E)$ are given in Eqs. (42), (84), and (85). The contribution from $\left(D^{0} \bar{D}^{0} \pi^{0}, D^{0} \bar{D}^{0} \gamma\right)$ is the sum of all the terms with the factor $\operatorname{Im} \kappa(E)$. Since the decay of $X(3872)$ into $J / \psi \pi^{+} \pi^{-}$is dominated by the decay into $J / \psi$ and a virtual $\rho^{0}$, this decay mode has isospin 1 . The contributions from all isospin-1 final states other than $D \bar{D} \pi$ and $D \bar{D} \gamma$ is the sum of all terms with the factor $\operatorname{Im} \gamma_{1}$. The contribution from $J / \psi \pi^{+} \pi^{-}$is obtained by replacing $\operatorname{Im} \gamma_{1}$ by a term $\left(\operatorname{Im} \gamma_{1}\right)^{J / \psi \pi^{+} \pi^{-}}$.

We will use several results from Table II to constrain the $B \rightarrow K$ transition coefficients. We will use the average values of the ratios $R_{0+}$ :

$$
\begin{aligned}
R_{0+}\left[J / \psi \pi^{+} \pi^{-}\right] & =0.63 \pm 0.17, \\
R_{0+}\left[D^{0} \bar{D}^{0} \pi^{0}, D^{0} \bar{D}^{0} \gamma\right] & =1.35 \pm 0.46 .
\end{aligned}
$$

We will also use the product of the branching fractions for $B^{+}$to decay into $K^{+}$and $D^{0} \bar{D}^{0} \pi^{0}$ or $D^{0} \bar{D}^{0} \gamma$ :

$$
\mathrm{Br}_{+}\left[D^{0} \bar{D}^{0} \pi^{0}, D^{0} \bar{D}^{0} \gamma\right]=(9.0 \pm 1.7) \times 10^{-5} .
$$

We will not use the product of the branching fractions for $B^{+}$to decay into $K^{+}$and $J / \psi \pi^{+} \pi^{-}$, because it would introduce the additional unknown parameter $\left(\operatorname{Im} \gamma_{1}\right)^{J / \psi \pi^{+} \pi^{-}}$.

We first consider the constraints on the parameters from $R_{0+}\left[J / \psi \pi^{+} \pi^{-}\right]$. Since $J / \psi \pi^{+} \pi^{-}$ has isospin 1 , the only resonant enhancement comes from the $X(3872)$ resonance just below the $D^{* 0} \bar{D}^{0}$ threshold. The dominant contribution to the product of branching fractions will come from the $D^{* 0} \bar{D}^{0}$ threshold region, where the line shape from the decay of $B^{+}$can be approximated by the simple expression in Eq. (88). The line shape from the decay of $B^{0}$ can be approximated by the same expression with the coefficients $C_{B^{+}{ }^{{ }^{+},}}$replaced by $C_{B^{0}}^{K^{0}, i}$. According to the isospin symmetry relations in Eqs. (75) and (83), this is equivalent to the substitutions $C_{B^{+}}^{K^{+}, 0} \rightarrow C_{B^{+}}^{K^{+}, 1}$ and $C_{B^{+}}^{K^{+}, 1} \rightarrow C_{B^{+}}^{K^{+}, 0}$. Thus the ratio $R_{0+}$ for the final state $J / \psi \pi^{+} \pi^{-}$is simply

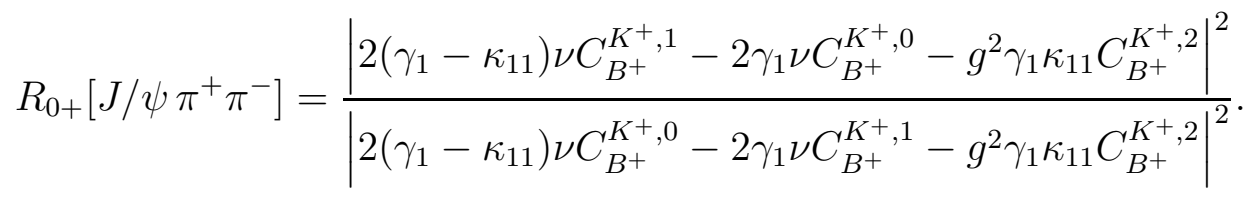


This ratio is different from 1 only if $C_{B^{+}}^{K^{+}, 1} \neq C_{B^{+}}^{K^{+}, 0}$, which means that the $I=1$ component of the amplitude for producing the charm mesons at short distance is nonzero. We will constrain this ratio to have the value in Eq. (98a).

If the line shape for $\left(D^{0} \bar{D}^{0} \pi^{0}, D^{0} \bar{D}^{0} \gamma\right)$ was completely dominated by the $X(3872)$ resonance below the $D^{* 0} \bar{D}^{0}$ threshold, the ratio $R_{0+}\left[D^{0} \bar{D}^{0} \pi^{0}, D^{0} \bar{D}^{0} \gamma\right]$ would also be given by the expression on the right side of Eq. (100). However the measured value of this ratio in Eq. (98b) is significantly larger than that for $J / \psi \pi^{+} \pi^{-}$in Eq. (98a). This is easy to understand. Both channels have a peak just below the $D^{* 0} \bar{D}^{0}$ threshold from the $X(3872)$ resonance. However the $\left(D^{0} \bar{D}^{0} \pi^{0}, D^{0} \bar{D}^{0} \gamma\right)$ channel also has a threshold enhancement above the $D^{* 0} \bar{D}^{0}$ threshold. At the peak of the threshold enhancement, which is very close to the threshold, the ratio in Eq. (100) may be a good approximation. However, above the peak in the threshold enhancement, the energy distribution decreases relatively slowly as $E$ increases. Because of this high-energy shoulder, there may be a substantial contribution from energies where the ratio $R_{0+}$ differs significantly from that in Eq. (100). Some idea of the energy range over which the threshold enhancement extends can be obtained from measurements of the $X(3872)$ resonance in the $D^{* 0} \bar{D}^{0}$ and $D^{0} \bar{D}^{* 0}$ decay channels by the Belle and Babar Collaborations $[15,16]$. The average of their measurements of the position is $2.1 \pm 1.2 \mathrm{MeV}$ above the $D^{* 0} \bar{D}^{0}$ threshold. The average of their measurements of the width is $3.5_{-1.0}^{+1.6} \mathrm{MeV}$. These measurements imply that most of the contribution to the threshold enhancement comes from the energy region below the $D^{*+} D^{-}$threshold at $\nu_{11}=8.1 \mathrm{MeV}$. These measurements of the position and the width in the $D^{* 0} \bar{D}^{0}$ channel should not be interpreted as the actual position and the width of the $X(3872)$ resonance for two reasons. First, the $X(3872)$ resonance and the $D^{* 0} \bar{D}^{0}$ threshold enhancement are smeared into a single peak by the experimental resolution. This explains why the measured width in the $D^{* 0} \bar{D}^{0}$ channel is larger than the upper bound in Eq. (54) from measurements in the $J / \psi \pi^{+} \pi^{-}$channel. This effect also biases the measurement of the position towards larger values. Second, in the analysis procedures in Refs. $[15,16]$, a $D^{0} \pi^{0}$ whose invariant mass is close to the mass of the $D^{* 0}$ is constrained to have an invariant mass exactly equal to $M_{* 0}$. This shifts $D^{0} \bar{D}^{0} \pi^{0}$ events just below the $D^{* 0} \bar{D}^{0}$ threshold to above the threshold, which further biases the measurement of the position towards larger values. These two biases explain why the measured resonance position in the $D^{* 0} \bar{D}^{0}$ channel is larger than that in Eq. (1) by more than two standard deviations.

Our prescription for the branching fraction $\mathrm{Br}_{+}$for the final state $\left(D^{0} \bar{D}^{0} \pi^{0}, D^{0} \bar{D}^{0} \gamma\right)$ is an integral over $E$ of the appropriate energy distribution:

$$
\mathrm{Br}_{+}\left[D^{0} \bar{D}^{0} \pi^{0}, D^{0} \bar{D}^{0} \gamma\right]=\frac{1}{\Gamma\left[B^{+}\right]} \int_{-\delta_{00}}^{+\nu_{11}} d E \frac{d \Gamma}{d E}\left[B^{+} \rightarrow K^{+}+\left(D^{0} \bar{D}^{0} \pi^{0}, D^{0} \bar{D}^{0} \gamma\right)\right]
$$

where $\Gamma\left[B^{+}\right]=4.02 \times 10^{-10} \mathrm{MeV}$ is the total width of the $B^{+}$. The integrand is the contribution to the inclusive energy distribution in Eq. (71) from terms with the factor $\operatorname{Im} \kappa(E)$. We have chosen the integration region somewhat arbitrarily to extend from the $D^{0} \bar{D}^{0} \pi^{0}$ threshold at $-\delta_{00}=-7.1 \mathrm{MeV}$ to the $D^{*+} D^{-}$threshold at $\nu_{11}=+8.1 \mathrm{MeV}$. We will constrain the branching fraction in Eq. (101) to have the value in Eq. (99).

Our prescription for the ratio $R_{0+}$ for $\left(D^{0} \bar{D}^{0} \pi^{0}, D^{0} \bar{D}^{0} \gamma\right)$ is the ratio of two integrals like the one in Eq. (101):

$$
R_{0+}\left[D^{0} \bar{D}^{0} \pi^{0}, D^{0} \bar{D}^{0} \gamma\right]=\frac{\int_{-\delta_{00}}^{+\nu_{11}} d E d \Gamma\left[B^{0} \rightarrow K^{0}+\left(D^{0} \bar{D}^{0} \pi^{0}, D^{0} \bar{D}^{0} \gamma\right)\right] / d E}{\int_{-\delta_{00}}^{+\nu_{11}} d E d \Gamma\left[B^{+} \rightarrow K^{+}+\left(D^{0} \bar{D}^{0} \pi^{0}, D^{0} \bar{D}^{0} \gamma\right)\right] / d E}
$$


The integrand in the numerator differs from that in the denominator by the substitutions $C_{B^{+}, 0}^{K^{+},} \rightarrow C_{B^{+}}^{K^{+}, 1}$ and $C_{B^{+}}^{K^{+}, 1} \rightarrow C_{B^{+}}^{K^{+}, 0}$. We will constrain the ratio in Eq. (102) to have the value in Eq. (98b).

\section{B. $B$ meson decays into $K+\chi_{c 1}^{\prime}$}

The $X(3872)$ could arise from a fine tuning of the $\mathrm{P}$-wave charmonium state $\chi_{c 1}^{\prime} \equiv \chi_{c 1}(2 P)$ to near the $D^{* 0} \bar{D}^{0}$ threshold. However the $\chi_{c 1}^{\prime}$ could also be a separate resonance above the $D^{*+} D^{-}$threshold. The Zero-Range+Resonance model of Section III D allows for both possibilities. If the mass of the $\chi_{c 1}^{\prime}$ is sufficiently far above the $D^{*+} D^{-}$threshold, the primary effect of the coupling of the $\chi_{c 1}^{\prime}$ to the charm mesons is through their contribution to its width. Thus information about the decay $B \rightarrow K+\chi_{c 1}^{\prime}$ in the absence of resonant interactions with charm mesons can be used to constrain the coefficients for the $B \rightarrow K$ transition.

In the decay $B \rightarrow K+\chi_{c 1}^{\prime}$, the momentum transferred to the mesons in the final state is about $1100 \mathrm{MeV}$. This might be large enough that the decay can be treated using factorization methods that separate the hard momentum scales comparable to or larger than the momentum transfer from the soft hadronic momentum scales. There have been several studies of factorization for the decays $B \rightarrow K+\chi_{c J}$, where $\chi_{c J} \equiv \chi_{c J}(1 P), J=0,1,2$, is the lowest multiplet of $\mathrm{P}$-wave charmonium states. One might expect the amplitude for this process to satisfy a simple factorization formula analogous to that for the decay of a $B$ meson into two light hadrons [45]. In that factorization formula, the factor associated with $\chi_{c J}$ is the light-front distribution amplitude for the charmonium state. At leading order in the relative velocity of the charm quarks, this factor is proportional to $R_{\chi_{c}}^{\prime}(0)$, the derivative of the radial wavefunction at the origin. However, Chao, Song, and collaborators discovered infrared divergences at next-to-leading order in $\alpha_{s}$ that are proportional to $\left(m_{c} / m_{b}\right)^{2}$ $[46,47]$. These infrared divergences signal that the factorization formula holds, at best, only up to corrections of order $\left(m_{c} / m_{b}\right)^{2}$. Beneke and Vernazza studied the factorization of the decay amplitude in the limit $m_{b}, m_{c} \rightarrow \infty$ with $m_{c} / m_{b}$ fixed [48]. They demonstrated that all infrared divergences at next-to-leading order in $\alpha_{s}$ can be absorbed into the matrix element of a color-octet operator. In the asymptotic limit in which $\chi_{c J}$ is a Coulombic charmonium state, the color-octet matrix element is proportional to $R_{\chi_{c}}^{\prime}(0)$, but in general it is an independent nonperturbative factor. Bodwin, Tormo and Lee studied the factorization of the decay amplitude in the limit $m_{b} \rightarrow \infty$ with $m_{c}$ fixed [49]. They proved the factorization to all orders in $\alpha_{s}$ up to corrections suppressed by $m_{c} / m_{b}$. Their factorization formula has the same structure as that for decays into two light mesons. The factors associated with the $\chi_{c J}$ are matrix elements of local operators in nonrelativistic QCD. The matrix element at leading order in the relative velocity of the charm quark is proportional to $R_{\chi_{c}}^{\prime}(0)$.

There have been attempts to calculate the branching fraction for $B^{+} \rightarrow K^{+}+\chi_{c 1}^{\prime}$. Meng, Gao, and Chao used a naive factorization formula with an infrared cutoff on the divergences at order $\alpha_{s}$ and obtained the branching fraction $1.8 \times 10^{-4}$ [50]. They also applied their method to the decay into $K^{+}+\chi_{c 1}$ and obtained essentially the same branching fraction, which is smaller by about a factor of 3 than the measured value. Liu and Wang calculated the branching fraction for $B^{+} \rightarrow K^{+}+\chi_{c 1}^{\prime}$ [51] using a perturbative QCD method that incorporates Sudakov effects [52]. Their result was $\left(7.9_{-3.8}^{+4.9}\right) \times 10^{-4}$. They did not calculate the corresponding result for $K^{+}+\chi_{c 1}$, so the accuracy of their method can not be judged by comparing with data. 
In the absence of any reliable calculations of the branching fraction for $B^{+} \rightarrow K^{+}+\chi_{c 1}^{\prime}$, we will use a simple phenomenological estimate. We scale the measured branching fraction for $B^{+} \rightarrow K^{+}+\chi_{c 1}$ by factors that take into account its dependence on the mass and the wavefunction of the charmonium state. The dependence on the mass $M_{\chi_{c 1}}$ is primarily through a multiplicative factor $\lambda^{3 / 2}\left(M_{B}, m_{K}, M_{\chi_{c 1}}\right)$ that comes from the phase-space integral and from the Lorentz structure of the decay amplitude. We assume that the dependence on the wave function comes primarily from a multiplicative factor $\left|R_{\chi_{c}}^{\prime}(0)\right|^{2}$, where $R_{\chi_{c}}^{\prime}(0)$ is the derivative of the radial wavefunction at the origin. The factor $\left|R_{\chi_{c}}^{\prime}(0)\right|^{2}$ for both the $2 P$ and $1 P$ multiplets has been calculated for four potential models in Ref. [53]. The ratio of this factor for $2 P$ and $1 P$ ranges from 0.97 to 1.42 . We interpret this as a ratio 1.20 with a theoretical error \pm 0.23 that can be added in quadrature with the experimental error in the branching fraction for $B^{+} \rightarrow K^{+}+\chi_{c 1}$. The measured branching fraction for $B^{+} \rightarrow K^{+}+\chi_{c 1}$ is $(4.9 \pm 0.5) \times 10^{-4}$. Multiplying by our two scaling factors, our estimate for the branching fraction is

$$
\operatorname{Br}\left[B^{+} \rightarrow K^{+}+\chi_{c 1}^{\prime}\right] \approx\left[(5.9 \pm 1.3) \times 10^{-4}\right] \frac{\lambda^{3 / 2}\left(M_{B}, m_{K}, M_{\chi_{c 1}^{\prime}}\right)}{\lambda^{3 / 2}\left(M_{B}, m_{K}, M_{\chi_{c 1}}\right)} .
$$

The last factor ranges from 0.53 if the $\chi_{c 1}^{\prime}$ is near the $D^{* 0} D^{0}$ threshold to 0.39 for $M_{\chi_{c 1}^{\prime}}=$ $4000 \mathrm{MeV}$.

Our estimate for the branching fraction in Eq. (103) is based only on information about charmonium states. Thus the appropriate limit of the Zero-Range+Resonance model in Sec. III D is the Flatté limit in which the charm mesons scatter only through their coupling to the resonance. In this limit, the partial width for $B^{+} \rightarrow K^{+}+\chi_{c 1}^{\prime}$ is given by Eq. (95), with the short-distance factor $\Gamma_{B}^{K}$ given by Eq. (72). The factor $\lambda^{3 / 2}\left(M_{B}, m_{K}, M_{* 0}+M_{0}\right)$ in $\Gamma_{B}^{K}$ came from taking the limit $E \rightarrow 0$ in $\lambda^{3 / 2}\left(M_{B}, m_{K}, M_{* 0}+M_{0}+E\right)$. This factor matches the factor $\lambda^{3 / 2}\left(M_{B}, m_{K}, M_{\chi_{c 1}^{\prime}}\right)$ in Eq. (103). Matching the other factors in Eqs. (95) and (103), we obtain the constraint

$$
\left|C_{B^{+}, 0}^{K^{+}}-C_{B^{+}}^{K^{+}, 1}+C_{B^{+}}^{K^{+}, 2}\right|^{2}=(8.1 \pm 1.8) \times 10^{-13} .
$$

\section{C. $B$ meson decays into $K+D^{* 0} \bar{D}^{0}$}

The invariant mass distributions of charm meson pairs in the decays $B \rightarrow K+$ $\left(D^{* 0} \bar{D}^{0}, D^{0} \bar{D}^{* 0}\right)$ have been measured by the Babar and Belle Collaborations from the $D^{* 0} \bar{D}^{0}$ threshold near $3872 \mathrm{MeV}$ up to $4000 \mathrm{MeV}[15,16]$. Measurements of the enhancement near the $D^{* 0} \bar{D}^{0}$ threshold, which is associated with the $X(3872)$, provide the constraints on $\mathrm{Br}_{+}\left[D^{0} \bar{D}^{0} \pi^{0}, D^{0} \bar{D}^{0} \gamma\right]$ and $R_{0+}\left[D^{0} \bar{D}^{0} \pi^{0}, D^{0} \bar{D}^{0} \gamma\right]$ described in Section VIA. The distributions from $3880 \mathrm{MeV}$ to $4000 \mathrm{MeV}$ provide additional constraints. In particular, they constrain the possibility of an additional resonance that could be associated with the $\chi_{c 1}^{\prime}$.

The Babar Collaboration used a data sample that consists of about $N_{B \bar{B}}=3.83 \times 10^{8}$ $B \bar{B}$ pairs [15]. They combined both $B^{+}$and $B^{0}$ decays and the $D^{0} \bar{D}^{0} \pi^{0}$ and $D^{0} \bar{D}^{0} \gamma$ decay channels into single measurements in each energy bin. Their efficiencies for $B^{+}$and $B^{0}$ decays were $\epsilon_{+}=4.3 \times 10^{-4}$ and $\epsilon_{0}=0.7 \times 10^{-4}$, respectively. The numbers of events were given in energy bins of $2.5 \mathrm{MeV}$. The Belle Collaboration used a data sample that consists of about $N_{B \bar{B}}=6.57 \times 10^{8} B \bar{B}$ pairs [16]. They combined $B^{+}$and $B^{0}$ decays into separate measurements for the $D^{0} \bar{D}^{0} \pi^{0}$ and $D^{0} \bar{D}^{0} \gamma$ decay channels in each energy bin. 


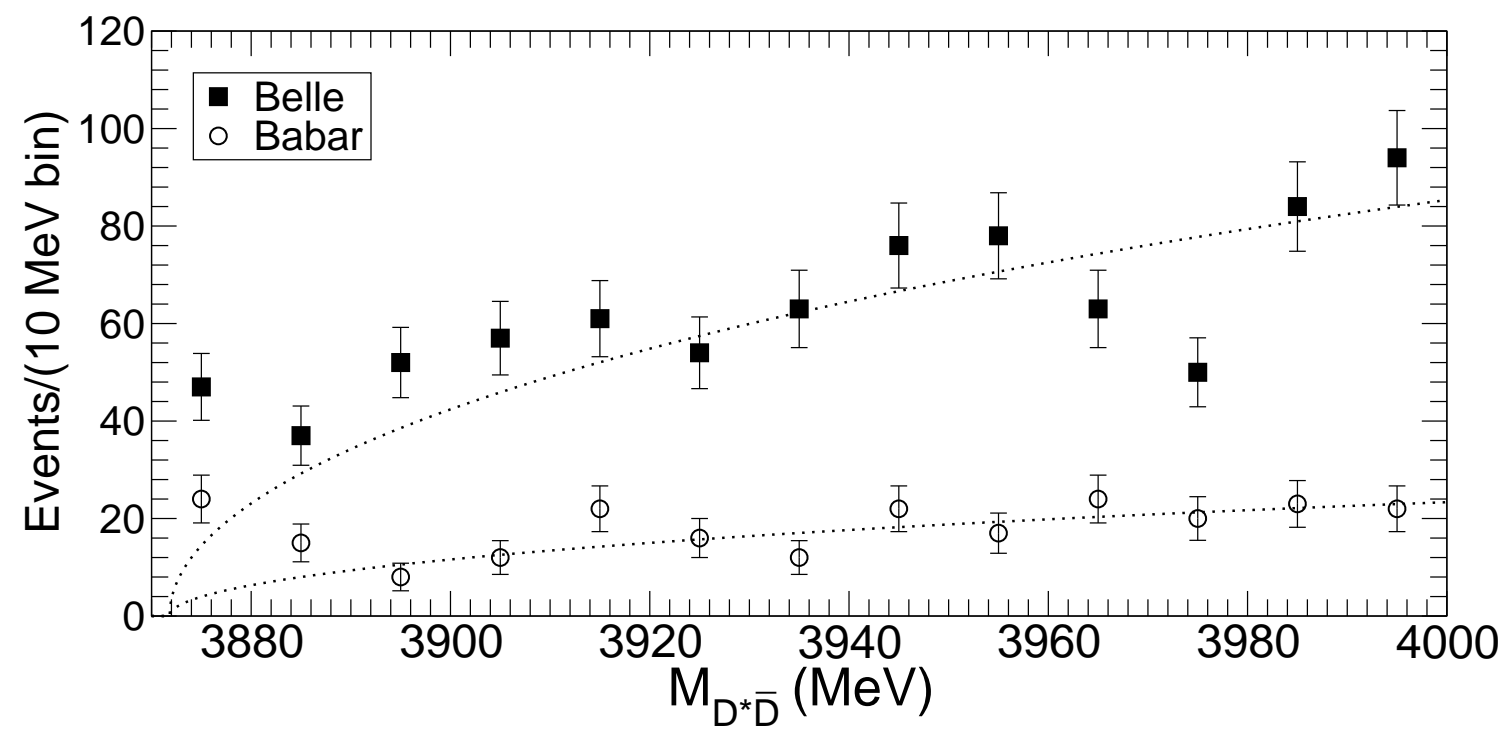

FIG. 1: $D^{* 0} \bar{D}^{0}$ invariant mass distributions from the decays $B \rightarrow K+\left(D^{* 0} \bar{D}^{0}, D^{0} \bar{D}^{* 0}\right)$. The data are the numbers of $\left(D^{* 0} \bar{D}^{0}, D^{0} \bar{D}^{* 0}\right)$ events per $10 \mathrm{MeV}$ bin observed by the Babar Collaboration [15] (open dots) and by the Belle Collaboration [16] (solid squares). The dotted curves are the best fits to the data in the bins from $3900 \mathrm{MeV}$ to $4000 \mathrm{MeV}$ with no signal and a background proportional to the phase-space volume.

From the efficiencies that were given, we infer that the efficiencies for $B^{+}$and $B^{0}$ decays were $\epsilon_{+}=3.93 \times 10^{-4}$ and $\epsilon_{0}=0.63 \times 10^{-4}$ in the $D^{0} \bar{D}^{0} \gamma$ channel and $\epsilon_{+}=4.24 \times 10^{-4}$ and $\epsilon_{0}=0.69 \times 10^{-4}$ in the $D^{0} \bar{D}^{0} \pi^{0}$ channel. The numbers of events were given in energy bins of $2 \mathrm{MeV}$. In the Belle analysis, the energy resolution increased from about $0.5 \mathrm{MeV}$ at $3880 \mathrm{MeV}$ to about $1 \mathrm{MeV}$ at $3900 \mathrm{MeV}$ and then to about $2 \mathrm{MeV}$ at $4000 \mathrm{MeV}$.

The Babar and Belle measurements provide no evidence for a second resonance above $3880 \mathrm{MeV}$, but they also do not exclude it. To simplify our analysis, we combine adjacent bins into bins with $10 \mathrm{MeV}$ width. The minimum number of events in any bin is then 8. This is large enough that the experimental uncertainty in a bin with $N$ events can be approximated by $\sqrt{N}$. The larger bins also decrease the sensitivity to the experimental energy resolution. We add the number of Belle events in the $D^{0} \bar{D}^{0} \pi^{0}$ and $D^{0} \bar{D}^{0} \gamma$ channels to obtain the total number of $\left(D^{0} \bar{D}^{0} \pi^{0}, D^{0} \bar{D}^{0} \gamma\right)$ events in each energy bin. The resulting Babar and Belle data sets are shown in Figure 1. The curves are the best fits to the data from $3900 \mathrm{MeV}$ to $4000 \mathrm{MeV}$ with no signal and with a background proportional to the phase-space volume. The large excesses in the first bin are associated with the $X(3872)$.

The predicted number of $D^{* 0} \bar{D}^{0}$ and $D^{0} \bar{D}^{* 0}$ events in an energy bin is the sum of the predicted numbers of signal events $N_{\text {sig }}$ and background events $N_{\text {bg. }}$. We consider only the energy bins above $3880 \mathrm{MeV}$, so there is a negligible overlap with the $X(3872)$ signal region, which is taken into account through the constraints on $\operatorname{Br}_{+}\left[D^{0} \bar{D}^{0} \pi^{0}, D^{0} \bar{D}^{0} \gamma\right]$ and $R_{0+}\left[D^{0} \bar{D}^{0} \pi^{0}, D^{0} \bar{D}^{0} \gamma\right]$ described in Section VI A. The predicted number of $\left(D^{0} \bar{D}^{0} \pi^{0}, D^{0} \bar{D}^{0} \gamma\right)$ signal events from both $B^{+}$decays and $B^{0}$ decays in an energy bin extending from $E_{1}$ to $E_{2}$ 
is

$$
\begin{aligned}
N_{\text {sig }}=\frac{N_{B \bar{B}}}{\Gamma\left[B^{+}\right]} \int_{E_{1}}^{E_{2}} d E( & \epsilon_{+} \frac{d \Gamma}{d E}\left[B^{+} \rightarrow K^{+}+\left(D^{0} \bar{D}^{0} \pi^{0}, D^{0} \bar{D}^{0} \gamma\right)\right] \\
& \left.+\epsilon_{0} \frac{d \Gamma}{d E}\left[B^{0} \rightarrow K^{0}+\left(D^{0} \bar{D}^{0} \pi^{0}, D^{0} \bar{D}^{0} \gamma\right)\right]\right)
\end{aligned}
$$

We take the background to be incoherent and proportional to the phase-space volumes for the two successive 2-body decays $B \rightarrow K+\left(D^{*} \bar{D}\right)$ and $\left(D^{*} \bar{D}\right) \rightarrow D^{* 0}+\bar{D}^{0}$. The predicted number of background events in an energy bin is

$$
N_{\mathrm{bg}}=C_{\mathrm{bg}} \int_{E_{1}}^{E_{2}} d E \lambda^{1 / 2}\left(M_{B}, M_{K}, M_{* 0}+M_{0}+E\right) \lambda^{1 / 2}\left(M_{* 0}+M_{0}+E, M_{* 0}, M_{0}\right),
$$

where $C_{\mathrm{bg}}$ is an adjustable constant that is different for the Babar data and for the Belle data.

The inclusive energy distribution $d \Gamma / d E$ for $B^{+}$decay is given by the factorization formula in Eq. (71). In the Zero-Range+Resonance model, the sums are over $i, j \in\{0,1,2\}$. The corresponding energy distribution for $B^{0}$ decay can be obtained by the interchange $C_{B^{+}}^{K^{+}, 0} \leftrightarrow$

$C_{B^{+}}^{K^{+}, 1}$. The energy-dependent functions $\operatorname{Im} F_{i j}(E)$ are expressed as linear combinations of the imaginary parts of $\kappa(E), \kappa_{1}(E), \gamma_{0}, \gamma_{1}$, and $\nu$ in Eqs. (42), (84), and (85). The energy distribution for $\left(D^{0} \bar{D}^{0} \pi^{0}, D^{0} \bar{D}^{0} \gamma\right)$ is the sum of the terms with the factor $\operatorname{Im} \kappa(E)$. The ZeroRange+Resonance model should give an accurate description of this energy distribution in the $D^{*} \bar{D}$ threshold region. We will use this model all the way up to $4000 \mathrm{MeV}$, which is $120 \mathrm{MeV}$ above the $D^{*+} D^{-}$threshold. At energies well above the $D^{*+} D^{-}$threshold, the resonance term in the amplitude may still be accurate, but the zero-range approximation to the non-resonance term becomes inadequate. Thus in the high energy region, our model for the non-resonance amplitude can at best be regarded as illustrative.

\section{NUMERICAL ANALYSIS}

In this section, we analyze the available data to determine whether they are able to discriminate between binding mechanisms for the $X(3872)$. Our strategy is to fix the interaction parameters of the Zero-Range+Resonance model and then to vary the $B \rightarrow K$ transition coefficients to obtain the best possible fit to all the constraints. We repeat this procedure for various values of the interaction parameters to see if there are any parameters that are preferred by the constraints.

Given a set of interaction parameters, we determine the $B \rightarrow K$ transition coefficients by minimizing the $\chi^{2}$ associated with the following constraints:

- the experimental value for $R_{0+}\left[J / \psi \pi^{+} \pi^{-}\right]$in Eq. (98a), which is set equal to the theoretical result in Eq. (100).

- the experimental value for $R_{0+}\left[D^{0} \bar{D}^{0} \pi^{0}, D^{0} \bar{D}^{0} \gamma\right]$ in Eq. (98b), which is set equal to the theoretical result in Eq. (102).

- the experimental value for $\operatorname{Br}_{+}\left[D^{0} \bar{D}^{0} \pi^{0}, D^{0} \bar{D}^{0} \gamma\right]$ in Eq. (99), which is set equal to the theoretical result in Eq. (101). 


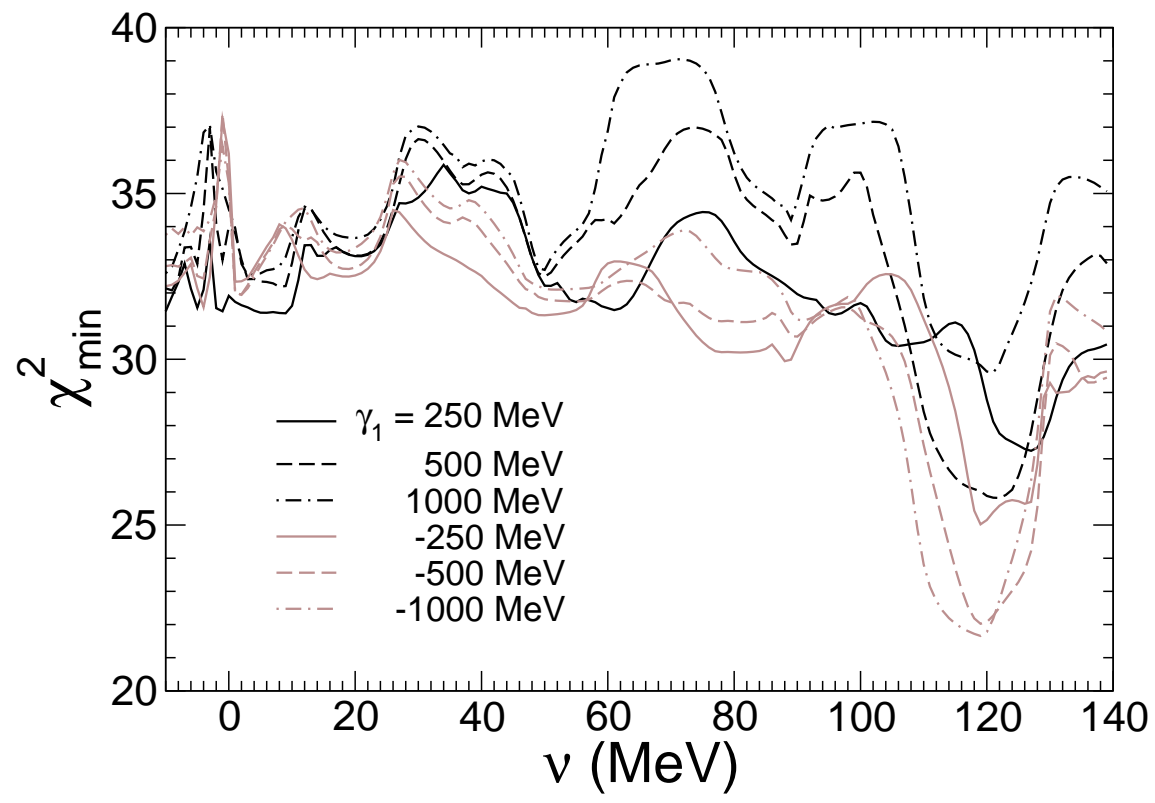

FIG. 2: The minimum of $\chi^{2}$ as a function of $\nu$ for $\gamma=28 \mathrm{MeV}, g=0.4$, and various values of $\gamma_{1}$.

- the constraint in Eq. (104), which follows from our phenomenological estimate for the branching fraction for $B^{+} \rightarrow K^{+}+\chi_{c 1}^{\prime}$.

- the numbers of $B \rightarrow K+\left(D^{* 0} \bar{D}^{0}, D^{0} \bar{D}^{* 0}\right)$ events observed by the Babar and Belle collaborations in each of the 12 energy bins covering the range 3880 to $4000 \mathrm{MeV}$. The two data sets are shown in Figure 1. The predicted number of events in an energy bin is the sum of the signal given by Eq. (105) and the background given by Eq. (106).

The total number of constraints is 28. The complex constants $C_{B^{+}}^{K^{+}, i}$ are determined up to an overall phase by 5 real parameters. The numbers of background events in the Babar and Belle data are determined by 2 additional parameters. These 7 parameters are then varied to minimize the $\chi^{2}$ associated with the 28 constraints.

In the Zero-Range+Resonance model, the independent interaction parameters are $\gamma, \gamma_{1}, g$, and $\nu$, with $\gamma_{0}$ given by Eq. (45). The allowed region for the real and imaginary parts of $\gamma$ are given in Eqs. (57) and (58). Our constraints on the energy distributions are insensitive to the value of $\gamma$, because they either involve integrals over the $D^{*} \bar{D}$ threshold region or they involve only higher energies. We therefore set the real part of $\gamma$ to its central value in Eq. (57) and its imaginary part to zero: $\gamma=28 \mathrm{MeV}$. The resonance coupling constant was determined in Eq. (59) to be $g=0.40$. The undetermined interaction parameters are the isovector inverse scattering length $\gamma_{1}$ and the resonance parameter $\nu$. Our constraints are insensitive to the imaginary parts of $\nu$ and $\gamma_{1}$, so we set them to 0 . We expect the isovector inverse scattering length $\gamma_{1}$ to be large compared to the momentum scale $\kappa_{11}=125 \mathrm{MeV}$ associated with isospin splittings. We will therefore consider values in the region $\left|\gamma_{1}\right|>2 \kappa_{11}=250 \mathrm{MeV}$. We consider values of $\nu$ in the range $-10 \mathrm{MeV}<\operatorname{Re} \nu<140 \mathrm{MeV}$. For $\nu<-10 \mathrm{MeV}$, there would be a second resonance below the $D^{* 0} \bar{D}^{0}$ threshold in addition to the $X(3872)$, which is not observed. For $\nu>140 \mathrm{MeV}$, the second resonance would be above $4000 \mathrm{MeV}$, beyond the region covered by the Belle and Babar data.

We have performed fits of the 7 parameters to the 28 constraints for $\gamma=28 \mathrm{MeV}, g=0.4$, and different values of $\nu$ and $\gamma_{1}$. The parameter $\nu$ has been varied from $-10 \mathrm{MeV}$ up to 140 
$\mathrm{MeV}$ in steps of $1 \mathrm{MeV}$. Since our results are less sensitive to $\gamma_{1}$, we have repeated the fit only for a few discrete values of $\gamma_{1}: \pm 250, \pm 500$ and $\pm 1000 \mathrm{MeV}$. The minimum values of $\chi^{2}$ for the various values of $\gamma_{1}$ are shown in Figure 2 as a function of $\nu$. For $\nu<100 \mathrm{MeV}$, the minimum $\chi^{2}$ lies in the range $30<\chi_{\min }^{2}<39$ for all values of $\gamma_{1}$, indicating that the quality of the fit is rather insensitive to $\gamma_{1}$ and $\nu$. The minimum value of $\chi_{\min }^{2}=21.7$ is reached at $\nu=119 \mathrm{MeV}$ and $\gamma_{1}=-1000 \mathrm{MeV}$. For these values of $\nu$ and $\gamma_{1}$, we verified that the fit and the value of $\chi_{\min }^{2}$ are insensitive to variations in the real and imaginary parts of $\gamma$. For $\nu>130 \mathrm{MeV}$, the minimum $\chi^{2}$ lies in the range $29<\chi_{\min }^{2}<36$, indicating again that the quality of the fit is rather insensitive to $\gamma_{1}$.

In Figure 3, we show the $D^{* 0} \bar{D}^{0}$ invariant mass distributions for $\gamma=28 \mathrm{MeV}, g=0.4$, and three pairs of parameters $\left(\nu, \gamma_{1}\right)$ that are approximate local minima of $\chi^{2}:(-10,250)$, $(50,-250)$, and $(120,-1000) \mathrm{MeV}$. The third pair of parameters is close to the global minimum of $\chi^{2}$. The predicted numbers of $D^{* 0} \bar{D}^{0}$ events in a $10 \mathrm{MeV}$ bin observed by the Belle (Babar) experiment can be obtained by averaging the upper (lower) solid line over the bin. The background contributions are shown as dotted lines. For the first set of parameters, the $X(3872)$ arises from a fine-tuning of the energy of the $\chi_{c 1}^{\prime}$. For the second and third sets of parameters, the $X(3872)$ arises from a fine-tuning of the strength of the interactions between $D^{* 0}$ and $\bar{D}^{0}$. For all three pairs of parameters, there is an $X(3872)$ resonance below the $D^{* 0} \bar{D}^{0}$ threshold and a $D^{* 0} \bar{D}^{0}$ threshold enhancement above the threshold. For the first set of parameters, there are no additional peaks in the energy distribution. For the second set of parameters, there is an additional peak near $3913 \mathrm{MeV}$ corresponding to the $\chi_{c 1}^{\prime}$ resonance. For the third set of parameters, there is a peak near $3983 \mathrm{MeV}$ corresponding to the $\chi_{c 1}^{\prime}$ resonance, but it is preceded by a sharp decrease in the number of events down to near the background level. This surprising behavior arises from destructive interference between the resonant and nonresonant amplitudes for producing charm meson pairs.

In Figure 4, we show the predicted energy distributions for the $\left(D^{0} \bar{D}^{0} \pi^{0}, D^{0} \bar{D}^{0} \gamma\right)$ signal and for the $\left(D^{+} \bar{D}^{0} \pi^{-}, D^{0} D^{-} \pi^{+}, D^{+} D^{-} \pi^{0}, D^{+} D^{-} \gamma\right)$ signal from both $B^{+}$decay and $B^{0}$ decay for the same three sets of parameters as in Figure 3 . The $\left(D^{+} \bar{D}^{0} \pi^{-}, D^{0} D^{-} \pi^{+}, D^{+} D^{-} \pi^{0}, D^{+} D^{-} \gamma\right)$ signal is the sum of the terms in the factorization formula with a factor of $\operatorname{Im} \kappa_{1}(E)$. The two signals correspond to $\left(D^{* 0} \bar{D}^{0}, D^{0} \bar{D}^{* 0}\right)$ and to $\left(D^{*+} D^{-}, D^{+} D^{*-}\right)$ above the appropriate thresholds. For all three sets of parameters, the energy distribution for $\left(D^{+} \bar{D}^{0} \pi^{-}, D^{0} D^{-} \pi^{+}, D^{+} D^{-} \pi^{0}, D^{+} D^{-} \gamma\right)$ has an $X(3872)$ resonance below the $D^{* 0} \bar{D}^{0}$ threshold, but there is no $D^{* 0} \bar{D}^{0}$ threshold enhancement just above the threshold. The resonance should be interpreted as a contribution from $D^{+} D^{-} \gamma$, because it is below the thresholds for $D^{+} \bar{D}^{0} \pi^{-}$and $D^{+} D^{-} \pi^{0}$. A quantitative treatment of this contribution would require using the energy-dependent width for the $D^{*+}$ defined in Ref. [9]. For the second set of parameters, there is a peak at the $\chi_{c 1}^{\prime}$ resonance near $3913 \mathrm{MeV}$ in both $D^{* 0} \bar{D}^{0}$ and $D^{*+} D^{-}$, but the peak is higher in $D^{*+} D^{-}$. For the third set of parameters, there is constructive interference in $D^{*+} D^{-}$at the $\chi_{c 1}^{\prime}$ resonance near $3980 \mathrm{MeV}$ in contrast to the destructive interference in $D^{* 0} \bar{D}^{0}$.

In Figure 5, we show the behavior of the line shapes in Figure 4 in the $D^{*} \bar{D}$ threshold region. At the $X(3872)$ resonance, the line shapes in $\left(D^{0} \bar{D}^{0} \pi^{0}, D^{0} \bar{D}^{0} \gamma\right)$ and in $D^{+} D^{-} \gamma$ coming from $B^{+}$decay and $B^{0}$ decay differ only in their normalizations. The line shapes in $\left(D^{0} \bar{D}^{0} \pi^{0}, D^{0} \bar{D}^{0} \gamma\right)$ have a $D^{* 0} \bar{D}^{0}$ threshold enhancement whose peak is about as far above the $D^{* 0} \bar{D}^{0}$ threshold as the $X(3872)$ resonance is below the threshold. The two maxima are well-separated, because we have set $\operatorname{Im} \gamma=0$. If $\operatorname{Im} \gamma$ is large enough, the resonance and the threshold enhancement will be smeared into a line shape with a single maximum. The 


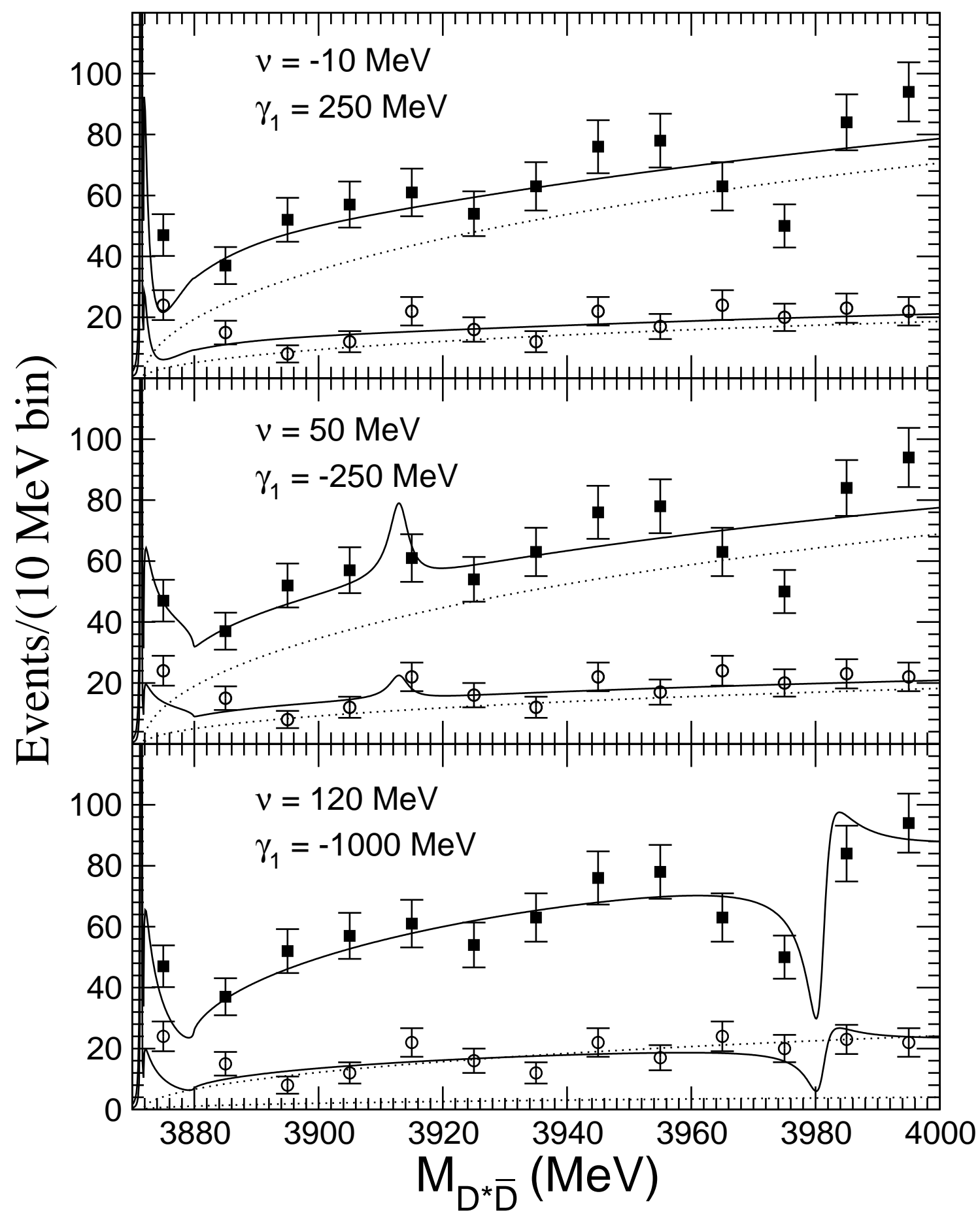

FIG. 3: Numbers of $B \rightarrow K+\left(D^{* 0} \bar{D}^{0}, D^{0} \bar{D}^{* 0}\right)$ events as functions of the invariant mass of the charm meson pair. The data are the numbers of events observed by the Babar Collaboration [15] (open circles) and by the Belle Collaboration [16] (solid squares) in $10 \mathrm{MeV}$ bins. The predicted $\left(D^{0} \bar{D}^{0} \pi^{0}, D^{0} \bar{D}^{0} \gamma\right)$ distributions for Belle (upper solid lines) and for Babar (lower solid lines) are shown for $\gamma=28 \mathrm{MeV}, g=0.4$, and three sets of values of $\left(\nu, \gamma_{1}\right)$. The background contributions are shown as dotted lines. 


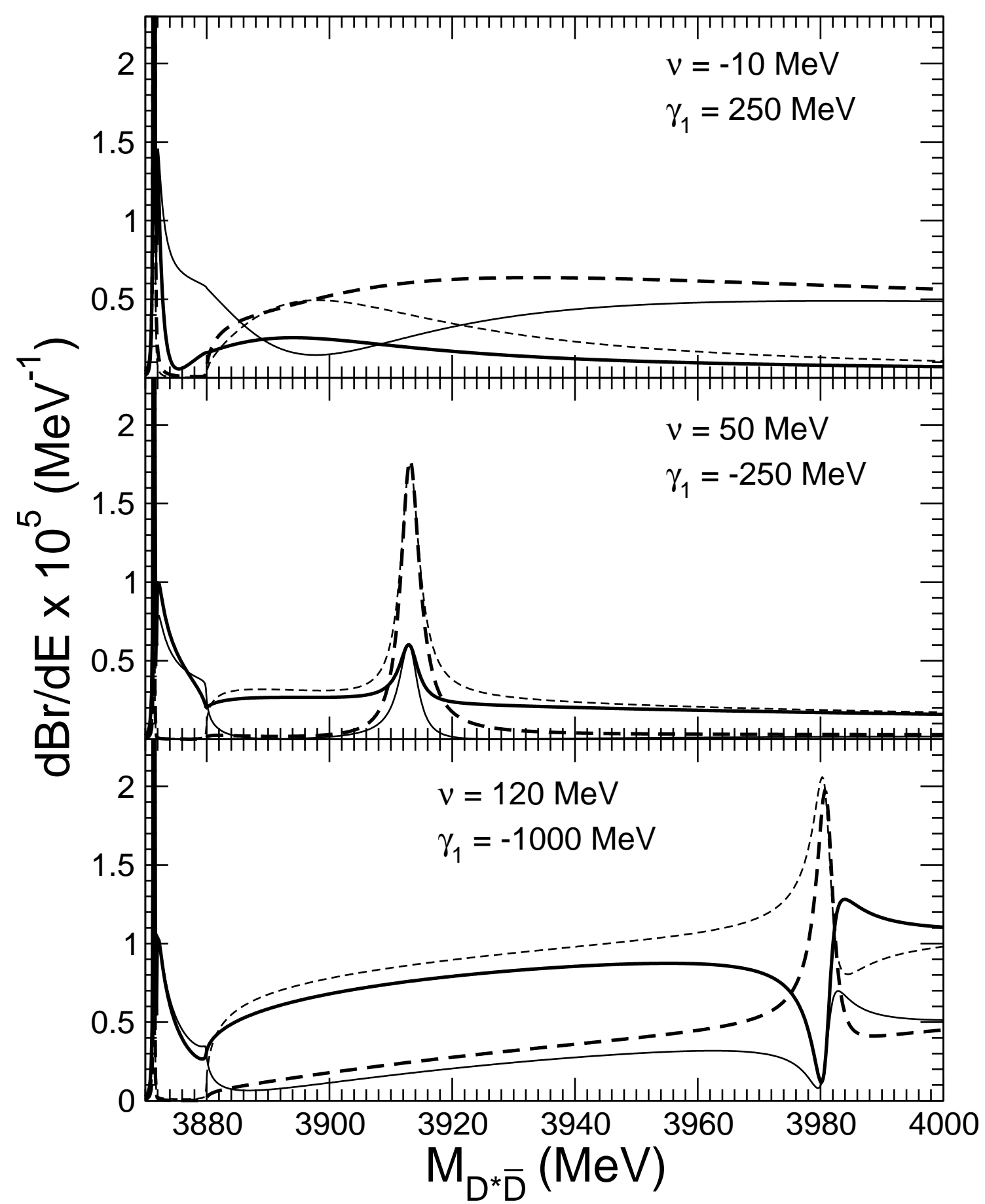

FIG. 4: Energy distributions for the $\left(D^{0} \bar{D}^{0} \pi^{0}, D^{0} \bar{D}^{0} \gamma\right)$ signal (solid lines) and for the $\left(D^{+} \bar{D}^{0} \pi^{-}, D^{0} D^{-} \pi^{+}, D^{+} D^{-} \pi^{0}, D^{+} D^{-} \gamma\right)$ signal (dashed lines) from both $B^{+}$decay (thicker lines) and $B^{0}$ decays (thinner lines) for $\gamma=28 \mathrm{MeV}, g=0.4$, and three sets of values of $\left(\nu, \gamma_{1}\right)$. 


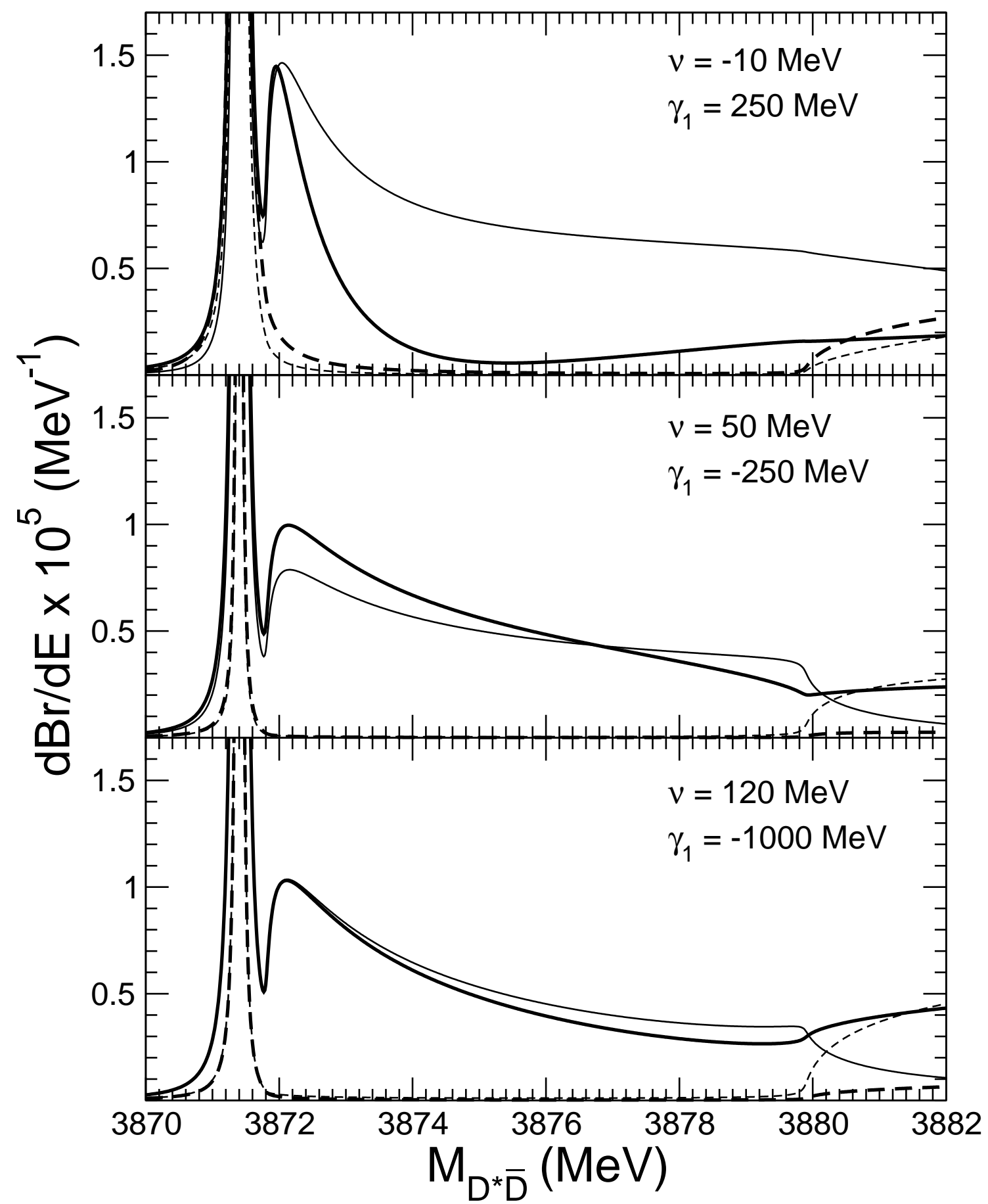

FIG. 5: Line shapes in the $D^{*} \bar{D}$ threshold region for the $\left(D^{0} \bar{D}^{0} \pi^{0}, D^{0} \bar{D}^{0} \gamma\right)$ signal (solid lines) and for the $\left(D^{+} \bar{D}^{0} \pi^{-}, D^{0} D^{-} \pi^{+}, D^{+} D^{-} \pi^{0}, D^{+} D^{-} \gamma\right)$ signal (dashed lines) from both $B^{+}$decay (thicker lines) and $B^{0}$ decays (thinner lines) for $\gamma=28 \mathrm{MeV}, g=0.4$, and three sets of values of $\left(\nu, \gamma_{1}\right)$. 
lines shapes from $B^{+}$decay and $B^{0}$ decay can be quite different in the region up to $D^{*+} D^{-}$ threshold, and they can change dramatically at that threshold. The energy distributions in $\left(D^{+} \bar{D}^{0} \pi^{-}, D^{0} D^{-} \pi^{+}, D^{+} D^{-} \pi^{0}, D^{+} D^{-} \gamma\right)$ are close to zero between the $D^{* 0} \bar{D}^{0}$ and $D^{*+} D^{-}$ thresholds, but they can increase dramatically above the $D^{*+} D^{-}$threshold.

\section{SUMMARY}

We have derived general line shapes for the $X(3872)$ that can be used to discriminate between alternative binding mechanisms. In the $D^{* 0} \bar{D}^{0}$ threshold region, which extends only to a few $\mathrm{MeV}$ from the threshold, the line shapes are universal in the sense that they are determined only by the binding energy and width of the $X(3872)$ [10]. In the $D^{*} \bar{D}$ threshold region, which extends to tens of $\mathrm{MeV}$ from the threshold, the line shapes depend on the binding mechanism. The mechanism could be the fine-tuning of the interaction between the charm mesons to near the critical strength for a bound state, in which case the appropriate line shapes are the zero-range line shapes of Ref. [10]. The mechanism could also be the fine-tuning of the energy of a resonance to near the $D^{* 0} \bar{D}^{0}$ threshold, in which case the appropriate line shapes are the Flatté line shapes. The Flatté line shapes are essentially those introduced in Ref. [20], except that one cannot ignore the essential contribution to the line shape in the $\left(D^{0} \bar{D}^{0} \pi^{0}, D^{0} \bar{D}^{0} \gamma\right)$ channel from the $X(3872)$ resonance peak below the threshold. Our general line shapes include the zero-range line shapes and the Flatté line shapes as special cases.

Our general line shapes take into account scattering between two coupled channels with the same reduced mass $\mu$. This is a good approximation if the masses of the pairs of particles in the two channels differ only by isospin splittings. These line shapes could be applied to any weakly-bound hadronic molecule that has S-wave couplings to a pair of thresholds separated by isospin splittings. However the complications associated with the coupled channels are only relevant if the binding energy of the molecule and the widths of the constituents are all much smaller than the isospin splittings, which are typically less than $10 \mathrm{MeV}$. The $X(3872)$ may be the unique hadron that satisfies this requirements. If these conditions are not satisfied, one might as well ignore the complications associated with the isospin splittings. The zero-range approximations for the direct interaction between the pair of mesons may also be inadequate in this case.

A crucial ingredient in our general line shapes are the coupled-channel scattering amplitudes for the neutral and charged charm meson channels $\left(D^{* 0} \bar{D}^{0}\right)_{+}$and $\left(D^{*+} D^{-}\right)_{+}$, which are given in Eqs. (39). The resonance propagator is given in Eq. (41). They depend on 4 interaction parameters: $\gamma_{0}, \gamma_{1}, \nu$, and $g$. The scattering amplitudes satisfy the constraints of unitarity exactly if these parameters are real. The analytic continuation of the parameters to complex values can be used to take into account some of the effects of states that are not treated explicitly. The line shapes in the universal region depend only on the inverse scattering length $\gamma$, which satisfies Eq. (43). It is therefore convenient to use Eq. (45) to eliminate $\gamma_{0}$ in favor of $\gamma$. The real and imaginary parts of $\gamma$ are constrained by measurements of the binding energy and width of the $X(3872)$. The constraints are given in Eqs. (57) and (58). We expect the isovector inverse scattering length $\gamma_{1}$ to be much larger than $\kappa_{1} \approx 125 \mathrm{MeV}$, but we do not have any useful quantitative constraints on this parameter. If the resonance is identified with the P-wave charmonium state $\chi_{c 1}^{\prime} \equiv \chi_{c 1}(2 P)$, charmonium phenomenology can be used to constrain the resonance parameters $\nu$ and $g$. The coupling constant $g$ is determined from phenomenological models of the decays $\chi_{c 1}^{\prime} \rightarrow D^{*} \bar{D}$ to have the value 
$g=0.4$ given in Eq. (59). The variations among potential models are sufficiently large that they do not provide any useful constraints on the energy parameter $\nu$.

The general line shapes for a weakly-bound hadronic molecule that is produced by a short-distance process can be expressed in terms of a factorization formula for the inclusive energy distribution. In the case of the $X(3872)$, an example of a short-distance process is a $B \rightarrow K$ transition with momentum transfer of about $1100 \mathrm{MeV}$, which can produce pairs of charm mesons in the $D^{*} \bar{D}$ threshold region. The factorization formula for the inclusive energy distribution for states with quantum numbers $1^{++}$that are produced by the $B^{+} \rightarrow K^{+}$transition is given in Eq. (71). All the dependence on the energy $E$ is in the long-distance factors $\operatorname{Im} F_{i j}(E)$. The short-distance coefficients $C_{B^{+}}^{K^{+}, i}$ are complex constants. The factorization formula for another short-distance process differs only in the values of the short-distance coefficients. The short-distance coefficients for the $B^{0} \rightarrow K^{0}$ transition are determined in terms of those for the $B^{+} \rightarrow K^{+}$transition by the isospin-symmetry relations in Eqs. (75) and (83). These short-distance coefficients are constrained by measurements of $B \rightarrow K+X(3872)$, such as those in Eqs. (98) and (99). The additional constraint in Eq. (104) was obtained from a phenomenological estimate of the decay rate for $B \rightarrow K+\chi_{c 1}^{\prime}$.

In the factorization formula for the inclusive energy distribution in Eq. (71), the longdistance functions $\operatorname{Im} F_{i j}(E)$ can be resolved into contributions from different decay channels of the $X(3872)$ by expressing them in forms consistent with the Cutkosky cutting rules. The functions $\operatorname{Im} F_{i j}(E)$ are expressed as linear combinations of the imaginary parts of the functions $\kappa(E)$ and $\kappa_{1}(E)$ and the imaginary parts of the parameters $\gamma_{0}, \gamma_{1}$, and $\nu$ in Eqs. (42), (84), and (85). The terms proportional to $\operatorname{Im} \kappa(E)$ can be interpreted as the contributions from $\left(D^{0} \bar{D}^{0} \pi^{0}, D^{0} \bar{D}^{0} \gamma\right)$. The terms proportional to $\operatorname{Im} \kappa_{1}(E)$ can be interpreted as the contributions from $\left(D^{+} \bar{D}^{0} \pi^{-}, D^{0} D^{-} \pi^{+}, D^{+} D^{-} \pi^{0}, D^{+} D^{-} \gamma\right)$. The terms proportional to $\operatorname{Im} \nu$, $\operatorname{Im} \gamma_{0}$, and $\operatorname{Im} \gamma_{1}$ can be interpreted as the contributions from other decay modes of $\chi_{c 1}^{\prime}$, other channels with isospin 0 , and other channels with isospin 1.

In Section VII, we used our line shapes to carry out a phenomenological analysis of the data on $B \rightarrow K+X(3872)$ and data from the Belle and Babar Collaborations on $B \rightarrow$ $K+D^{* 0} \bar{D}^{0}$, with the $D^{* 0} \bar{D}^{0}$ invariant mass ranging up to $4000 \mathrm{MeV}$. We assumed that the binding mechanism for the $X(3872)$ is either a fine-tuning of the strength of the interaction between the charm mesons or a fine-tuning of the energy of the P-wave charmonium state $\chi_{c 1}^{\prime}$. We extrapolated our line shapes for the $D^{*} \bar{D}$ threshold region all the way up to $128 \mathrm{MeV}$ above the $D^{* 0} \bar{D}^{0}$ threshold. In the high energy region, the nonresonant contributions to the production amplitudes, which were derived using a zero-range approximation, can at best be regarded as an illustrative model. We found that the two mechanisms for the binding of the $X(3872)$ are both compatible with the data for $B \rightarrow K+D^{* 0} \bar{D}^{0}$ and our other constraints. One way to exclude the tuning of the $\chi_{c 1}^{\prime}$ energy as a binding mechanism for the $X(3872)$ is to observe the $\chi_{c 1}^{\prime}$ resonance as a separate peak in the invariant mass distribution for $D^{* 0} \bar{D}^{0}$ and $D^{*+} D^{-}$. In the Belle and Babar data, there is no obvious peak in the $D^{* 0} \bar{D}^{0}$ invariant mass distribution between $3880 \mathrm{MeV}$ and $4000 \mathrm{MeV}$. However, the width of this peak is determined by the resonance parameters $\nu$ and $g$ and its height is determined by the short-distance coefficients $C_{B^{+}, i}^{K^{+}}$. Our calculations show that a $\chi_{c 1}^{\prime}$ resonance in this region is compatible with the data for $B \rightarrow K+D^{* 0} \bar{D}^{0}$ and with our constraints on the short-distance coefficients. An interesting possibility for this $\chi_{c 1}^{\prime}$ resonance is to have destructive interference between the resonant and nonresonant amplitudes in the $D^{* 0} \bar{D}^{0}$ decay channel and constructive interference in the $D^{*+} D^{-}$decay channel. This possibility is actually realized for the parameters that minimize the $\chi^{2}$ for our constraints, which give 
a $\chi_{c 1}^{\prime}$ resonance near $3980 \mathrm{MeV}$.

An alternative way to exclude the $\chi_{c 1}^{\prime}$ resonance mechanism for the binding of the $X(3872)$ is to calculate the $\chi_{c 1}^{\prime}$ mass using lattice gauge theory. The masses of excited charmonium states and states in the $c \bar{c}$ meson spectrum with exotic quantum numbers have been calculated by Dudek et al. using lattice gauge theory without dynamical light quarks [54]. The masses of the $2 P$ charmonium multiplet are significantly higher than those of the $1 D$ multiplet. Their analysis suggests that the $2^{++} c \bar{c}$ meson discovered near $3930 \mathrm{MeV}$ is more likely to be the ${ }^{3} D_{2}$ ground state than the first radial excitation of the ${ }^{3} P_{2}$. This suggests that the $2 P$ multiplet, including $\chi_{c 1}^{\prime}$, has higher mass. If these results are confirmed by lattice calculations with dynamical light quarks, it would exclude the $\chi_{c 1}^{\prime}$ resonance mechanism. Other resonance mechanisms, such as the tuning of the energy of a $1^{++}$tetraquark $c \bar{c}$ meson, are not easily constrained by phenomenology, but they can also be ultimately ruled out using lattice QCD calculations.

We used charmonium phenomenology to constrain the resonance parameters $g$ and $\nu$. Phenomenological estimates for the scattering parameters $\gamma_{0}$ and $\gamma_{1}$ could be obtained from meson potential models, such as those in Refs. [25, 38-42], or from meson scattering models, such as the one in Ref. [43]. Thus far, these models have been used primarily to calculate binding energies. They could also be used to calculate scattering variables, such as $\gamma_{0}$ and $\gamma_{1}$. A particularly convenient pair of scattering variables are the residues $Z_{0}$ and $Z_{1}$ of the poles in the elastic scattering amplitudes $f_{00}(E)$ for $D^{* 0} \bar{D}^{0}$ and $f_{11}(E)$ for $D^{*+} D^{-}$at the $X(3872)$ resonance. For the Zero-Range+Resonance model, the ratio $Z_{1}^{1 / 2} / Z_{0}^{1 / 2}$ is given in Eq. (47). It does not depend on the resonance parameters $g$ and $\nu$, so it is determined primarily by $\gamma_{1}$.

Our general line shapes could be used by experimentalists to carry out a global analysis of the energy distributions for the decay modes of the $X(3872)$ that is not biased towards a specific binding mechanism. One complication is the number of independent parameters in the line shapes. The line shapes in $\left(D^{0} \bar{D}^{0} \pi^{0}, D^{0} \bar{D}^{0} \gamma\right)$ depend essentially on 5 interaction parameters: the real parameters $\gamma_{1}, g$, and $\nu$ and the complex parameter $\gamma$. For other decay channels, there is also a normalization parameter, such as $\left(\operatorname{Im} \gamma_{1}\right)^{J / \psi \pi^{+} \pi^{-}}$for $J / \psi \pi^{+} \pi^{-}$and $\left(\operatorname{Im} \gamma_{0}\right)^{J / \psi \pi^{+} \pi^{-} \pi^{0}}$ for $J / \psi \pi^{+} \pi^{-} \pi^{0}$. The imaginary parts of $\gamma_{0}, \gamma_{1}$, and $\nu$ give contributions to $\operatorname{Im} \gamma$, as indicated by Eq. (46), but their effects should otherwise be negligible in the $D^{*} \bar{D}$ threshold region. In addition to the interaction parameters, there are 5 real parameters associated with the short-distance coefficients for the production of the charm meson pairs and the resonance. In an analysis of the line shapes of the $X(3872)$ produced by $B$ decays, the determination of the $5+$ interaction parameters and the 5 short-distance parameters would require analyzing several decay channels in both $B^{+}$and $B^{0}$ decays.

An alternative strategy would be to carry out two separate analyses assuming either the dynamical mechanism or the resonance mechanism for the binding of the $X(3872)$. With the dynamical mechanism, one could use the zero-range line shapes, which have $3+$ interaction parameters and 3 short-distance parameters. With the resonance mechanism, one could use the Flatté line shapes, which have $3+$ interaction parameters and 1 short-distance parameter. If one set of line shapes gives a significantly better global fit to the data, it would be evidence in favor of the corresponding binding mechanism for the $X(3872)$. 


\section{Acknowledgments}

This research was supported in part by the Department of Energy under grant DE-FG0291-ER40690 and by a joint grant from the Army Research Office and the Air Force Office of Scientific Research. One of us (E.B.) would like to thank C. Hanhart for valuable discussions.

\section{Appendix: Renormalization of the Zero-Range+Resonance Model}

The Zero-Range+Resonance model considered in Sections III D and VE can be derived from a renormalizable quantum field theory with local interactions. In this Appendix, we consider the renormalization of the interactions of this field theory and the renormalization of the local operators that describe production by a short-distance process.

\section{A. Renormalization of the Interactions}

The Zero-Range+Resonance model can be represented by a nonrelativistic field theory. The fields are scalar fields for the spin-0 charm mesons $D^{0}, \bar{D}^{0}, D^{+}$, and $D^{-}$, vector fields for the spin-1 charm mesons $D^{* 0}, \bar{D}^{* 0}, D^{*+}$, and $D^{*-}$, and a vector field for the resonance $\chi$. The vector fields, such as $\chi^{m}$, have a Cartesian vector index $m$. The interaction terms in the Hamiltonian density are

$$
\mathcal{H}_{\text {int }}=\frac{2 \pi}{\mu}\left(D^{*} \bar{D}\right)^{m \dagger} \Lambda_{0}\left(D^{*} \bar{D}\right)^{m}+\sqrt{\frac{2 \pi}{\mu}}\left[\left(D^{*} \bar{D}\right)^{m \dagger} G_{0} \chi^{m}+\chi^{m \dagger} G_{0}^{T}\left(D^{*} \bar{D}\right)^{m}\right]+\nu_{0} \chi^{m \dagger} \chi^{m} .
$$

where $\left(D^{*} \bar{D}\right)^{m}$ is a 2-component column vector with a Cartesian vector index $m$. Its upper and lower entries are the combinations of fields that annihilate pairs of charm mesons in the neutral channel $\left(D^{* 0} \bar{D}^{0}\right)_{+}$and in the charged channel $\left(D^{*+} D^{-}\right)_{+}$given in Eqs. (2). The bare parameters are the three independent entries of the symmetric matrix $\Lambda_{0}$, the two entries of the column vector $G_{0}$, and $\nu_{0}$. The factors of $\sqrt{2 \pi / \mu}$ in the Hamiltonian density have been inserted to simplify the renormalization equations. The $\chi^{m \dagger} \chi^{m}$ term has been included in the interaction Hamiltonian, because its coefficient $\nu_{0}$ requires renormalization.

The matrix of transition amplitudes $\mathcal{A}(E)$ for charm meson pairs in the channels $\left(D^{* 0} \bar{D}^{0}\right)_{+}$and $\left(D^{*+} D^{-}\right)_{+}$can be calculated by summing diagrams constructed out of the two types of vertices, resonance propagators, and loop subdiagrams involving pairs of charm mesons. The ultraviolet divergences in the loop diagrams can be regularized with an ultraviolet momentum cutoff $\Lambda_{\mathrm{UV}}$. The matrix $\mathcal{A}(E)$ can also be derived by solving the Lippmann-Schwinger integral equation, as described in Ref. [55]. It is convenient to express the solution in terms of the matrix of scattering amplitudes $f(E)$ defined by Eq. (12), which differs from $\mathcal{A}(E)$ by a factor of $\mu /(2 \pi)$. The solution for the inverse of $f(E)$ is

$$
f(E)^{-1}=-\left(\Lambda_{0}+G_{0} \frac{1}{E-\nu_{0}} G_{0}^{T}\right)^{-1}-\frac{2}{\pi} \Lambda_{\mathrm{UV}} I+K(E),
$$

where $I$ is the identity matrix and $K(E)$ is the diagonal matrix given in Eq. (14). The energy dependence on the right side of Eq. (108) allows $f(E)^{-1}$ to be expressed in the form in Eq. (35), in which the dependence on $\Lambda_{\mathrm{UV}}$ has been absorbed into renormalized parameters. 
The renormalized parameters are the three independent entries of the symmetric matrix $\Lambda$, the two entries of the column vector $G$, and $\nu$. The relations between the renormalized parameters and the bare parameters are given by

$$
\begin{aligned}
\Lambda & =Z^{-1} \Lambda_{0} \\
G & =Z^{-1} G_{0} \\
\nu & =\nu_{0}-G_{0}^{T}\left(1-Z^{-1}\right) \Lambda_{0}^{-1} G_{0}
\end{aligned}
$$

where the renormalization matrix $Z$ is

$$
Z=I+\frac{2}{\pi} \Lambda_{\mathrm{UV}} \Lambda_{0}
$$

The verification of the equality between the expressions for $f(E)^{-1}$ in Eqs. (108) and (35) can be simplified by taking advantage of the existence of renormalization-invariant combinations of parameters. Rewriting Eq. (109a) as $Z^{-1}=\Lambda \Lambda_{0}^{-1}$ and inserting it into Eqs. (109b) and $(109 \mathrm{c})$, we find that the following combinations of parameters are renormalization invariants:

$$
\begin{aligned}
\Lambda_{0}^{-1} G_{0} & =\Lambda^{-1} G \\
G_{0}^{T} \Lambda_{0}^{-1} & =G^{T} \Lambda^{-1}, \\
\nu_{0}-G_{0}^{T} \Lambda_{0}^{-1} G_{0} & =\nu-G^{T} \Lambda^{-1} G .
\end{aligned}
$$

The following function of $E$ is also a renormalization invariant:

$$
\left(\Lambda_{0}+G_{0} \frac{1}{E-\nu_{0}} G_{0}^{T}\right)^{-1}-\Lambda_{0}^{-1}=\left(\Lambda+G \frac{1}{E-\nu} G^{T}\right)^{-1}-\Lambda^{-1} .
$$

This can be verified by multiplying both sides on the left by $\Lambda_{0}+G_{0} G_{0}^{T} /\left(E-\nu_{0}\right)$ and on the right by $\Lambda+G G^{T} /(E-\nu)$ and then using the renormalization invariants in Eqs. (111). The equality between the expressions for $f(E)^{-1}$ in Eqs. (108) and (35) follows immediately from Eq. (112).

The complete propagator for the resonance $\chi$ can be obtained by summing the geometric series of self-energy corrections. The self-energy can be obtained by summing a geometric series of one-loop diagrams:

$$
\Sigma(E)=-G_{0}^{T}\left(\frac{2}{\pi} \Lambda_{\mathrm{UV}} I-K(E)\right)\left[I+\Lambda_{0}\left(\frac{2}{\pi} \Lambda_{\mathrm{UV}} I-K(E)\right)\right]^{-1} G_{0} .
$$

The complete resonance propagator is therefore

$$
P(E)=\left[E-\nu_{0}+G_{0}^{T}\left(\frac{2}{\pi} \Lambda_{\mathrm{UV}} I-K(E)\right)\left(\Lambda_{0}^{-1}+\frac{2}{\pi} \Lambda_{\mathrm{UV}} I-K(E)\right)^{-1} \Lambda_{0}^{-1} G_{0}\right]^{-1}
$$

This can be expressed in the form

$$
P(E)=\left[E-\nu_{0}+G_{0}^{T} \Lambda_{0}^{-1} G_{0}-G_{0}^{T} \Lambda_{0}^{-1}\left(\Lambda^{-1}-K(E)\right)^{-1} \Lambda_{0}^{-1} G_{0}\right]^{-1} .
$$

By using the renormalization invariants in Eqs. (111), this propagator can be expressed in terms of renormalized parameters and then simplified to the renormalized expression in Eq. (36). 


\section{B. Renormalization of the Production Operators}

The production of particles at short distances can be represented in an effective field theory by local operators that create the particles when acting on the vacuum. The matrix elements of the local operators are in general ultraviolet divergent. The corresponding renormalized operators are linear combinations that have finite matrix elements. The renormalized operators can be determined by calculating matrix elements of the local operators and then constructing linear combinations whose matrix elements are finite. Alternatively, the renormalized operators can be deduced by inspired guesswork.

In the Zero-Range+Resonance model, the particle that are produced at short distances can be pairs of charm mesons or the resonance. There are three leading operators: the two components of the column vector $\left(D^{*} \bar{D}\right)^{m}$ that appears in the interaction Hamiltonian in Eq. (107) and the resonance field $\chi^{m}$. They all have a Cartesian vector index $m$. We denote the corresponding renormalized operators by $\mathcal{O}_{0}^{m}, \mathcal{O}_{1}^{m}$, and $\mathcal{O}_{2}^{m}$. The Green's function $F_{i j}(E)$ for pairs of the operators $\mathcal{O}_{i}^{m}$ are defined in Eq. (70). The Green's function for a pair of operators $\chi^{m}$ is just $-P(E)$, where $P(E)$ is the resonance propagator in Eq. (114). Since this propagator can be expressed in the renormalized form in Eq. (36), the resonance field has finite matrix elements. We can therefore choose its hermitian conjugate as one of the renormalized operators:

$$
\mathcal{O}_{2}^{m \dagger}=\chi^{m}
$$

The matrix elements of $\left(D^{*} \bar{D}\right)^{m}$ are ultraviolet divergent. The renormalized operators $\mathcal{O}_{0}^{m}$ and $\mathcal{O}_{1}^{m}$ are linear combinations of the hermitian conjugates of all three local operators. A particular convenient choice consists of the operators obtained by differentiating $\mathcal{H}_{\text {int }}$ with respect to the components of $\left(D^{*} \bar{D}\right)^{m}$. The Green's functions for these operators are proportional to the scattering amplitudes. Our choices for the last two renormalized operators are

$$
\left(\begin{array}{c}
\mathcal{O}_{0}^{m \dagger} \\
\mathcal{O}_{1}^{m \dagger}
\end{array}\right)=\sqrt{\frac{2 \pi}{\mu}} \Lambda_{0}\left(D^{*} \bar{D}\right)^{m}+G_{0} \chi^{m}
$$

The normalization factor has been chosen so that the Green's functions for these operators are exactly equal to the scattering amplitudes $f_{i j}(E)$ for $i, j \in\{0,1\}$. The remaining Green's function $F_{i 2}(E)$ for $i=0,1$ are then given by

$$
F_{i 2}(E)=\left(f(E)\left[\left(E-\nu_{0}\right) \Lambda_{0}+G_{0} G_{0}^{T}\right]^{-1} G_{0}\right)_{i},
$$

where $f(E)$ is the matrix of scattering amplitudes. The corresponding renormalized expression is obtained by replacing $\nu_{0}, \Lambda_{0}$, and $G_{0}$ by $\nu, \Lambda$, and $G$. The equality of the two expressions for $F_{i 2}(E)$ follows from the fact that $\Lambda_{0}^{-1} G_{0}=\Lambda^{-1} G$ is an eigenvector of both $\nu_{0} I-\Lambda_{0}^{-1} G_{0} G_{0}^{T}$ and $\nu I-\Lambda^{-1} G G^{T}$ :

$$
\begin{aligned}
\left(\nu_{0} I-\Lambda_{0}^{-1} G_{0} G_{0}^{T}\right) \Lambda_{0}^{-1} G_{0} & =\left(\nu_{0}-G_{0}^{T} \Lambda_{0}^{-1} G_{0}\right) \Lambda_{0}^{-1} G_{0} \\
\left(\nu I-\Lambda^{-1} G G^{T}\right) \Lambda^{-1} G & =\left(\nu-G^{T} \Lambda^{-1} G\right) \Lambda^{-1} G .
\end{aligned}
$$

The Green's functions $F_{i j}(E)$ for $i, j \in\{0,1\}$ are not exactly equal to the scattering amplitudes $f_{i j}(E)$. The diagrams contributing to the $F_{i j}(E)$ are the same as the diagrams for $f_{i j}(E)$ except that the leading order diagram for scattering of charm meson pairs is 
omitted. Thus $F_{i j}(E)$ actually differs from $f_{i j}(E)$ by the additive constant $\left(\Lambda_{0}\right)_{i j}$. This constant gives an energy-independent contribution to the inclusive energy distribution in Eq. (71). This additive contribution can be interpreted as a constant background in decay channels of the $X(3872)$ that are not treated explicitly.

The renormalized operators $\mathcal{O}_{0}^{m}$ and $\mathcal{O}_{1}^{m}$ defined by Eq. (117) have different dimensions from the renormalized operator $\mathcal{O}_{2}^{m}=\chi^{m}$. For some purposes, it is more convenient for all the renormalized operators to have the same dimensions. One way to arrange for $\mathcal{O}_{2}^{m}$ to have the same dimension as $\mathcal{O}_{0}^{m}$ and $\mathcal{O}_{1}^{m}$ is to multiply $\chi^{m}$ by a constant with the appropriate dimension. In the text, the multiplying factor is chosen to be $\left(G^{T} G / 2\right)^{1 / 2}$, where $G$ is the two-component vector of renormalized interaction parameters. With this choice, the renormalized expressions for $F_{22}(E)$ and $F_{i 2}(E)$ are given by Eqs. (80) and (81).

[1] S. K. Choi et al. [Belle Collaboration], Phys. Rev. Lett. 91, 262001 (2003) [arXiv:hepex/0309032].

[2] B. Aubert et al. [BABAR Collaboration], Phys. Rev. D 74, 071101 (2006) [arXiv:hepex/0607050].

[3] K. Abe et al. [Belle Collaboration], arXiv:hep-ex/0505037.

[4] B. Aubert et al. [BABAR Collaboration], Phys. Rev. Lett. 102, 132001 (2009) [arXiv:0809.0042 [hep-ex]].

[5] A. Abulencia et al. [CDF Collaboration], Phys. Rev. Lett. 98, 132002 (2007) [arXiv:hepex/0612053].

[6] K. Abe et al. [Belle Collaboration], arXiv:hep-ex/0505038.

[7] G. Gokhroo et al. [Belle Collaboration], Phys. Rev. Lett. 97, 162002 (2006) [arXiv:hepex/0606055].

[8] P. del Amo Sanchez et al. [BABAR Collaboration], arXiv:1005.5190 [hep-ex].

[9] E. Braaten and M. Lu, Phys. Rev. D 76, 094028 (2007) [arXiv:0709.2697 [hep-ph]].

[10] E. Braaten and M. Lu, Phys. Rev. D 77, 014029 (2008) [arXiv:0710.5482 [hep-ph]].

[11] V. M. Abazov et al. [D0 Collaboration], Phys. Rev. Lett. 93, 162002 (2004) [arXiv:hepex/0405004].

[12] B. Aubert et al. [BABAR Collaboration], Phys. Rev. D 77, 111101 (2008) [arXiv:0803.2838 [hep-ex]].

[13] I. Adachi et al. [Belle Collaboration], arXiv:0809.1224 [hep-ex].

[14] T. Aaltonen et al. [CDF Collaboration], Phys. Rev. Lett. 103, 152001 (2009) [arXiv:0906.5218 [hep-ex]].

[15] B. Aubert et al. [BABAR Collaboration], Phys. Rev. D 77, 011102 (2008) [arXiv:0708.1565 [hep-ex]].

[16] T. Aushev et al. [Belle Collaboration], Phys. Rev. D 81, 031103 (2010) [arXiv:0810.0358 [hep-ex]].

[17] C. Hanhart, Yu. S. Kalashnikova and A. V. Nefediev, Phys. Rev. D 81, 094028 (2010) [arXiv:1002.4097 [hep-ph]].

[18] D. Acosta et al. [CDF II Collaboration], Phys. Rev. Lett. 93, 072001 (2004) [arXiv:hepex/0312021].

[19] B. Aubert et al. [BABAR Collaboration], Phys. Rev. D 71, 071103 (2005) [arXiv:hepex/0406022]. 
[20] C. Hanhart, Yu. S. Kalashnikova, A. E. Kudryavtsev and A. V. Nefediev, Phys. Rev. D 76, 034007 (2007) [arXiv:0704.0605 [hep-ph]].

[21] O. Zhang, C. Meng and H. Q. Zheng, Phys. Lett. B 680, 453 (2009) [arXiv:0901.1553 [hep-ph]].

[22] E. Braaten and J. Stapleton, Phys. Rev. D 81, 014019 (2010) [arXiv:0907.3167 [hep-ph]].

[23] Yu. S. Kalashnikova and A. V. Nefediev, Phys. Rev. D 80, 074004 (2009) [arXiv:0907.4901 [hep-ph]].

[24] M. B. Voloshin, Phys. Rev. D 76, 014007 (2007) [arXiv:0704.3029 [hep-ph]].

[25] Y. R. Liu, X. Liu, W. Z. Deng and S. L. Zhu, Eur. Phys. J. C 56, 63 (2008) [arXiv:0801.3540 [hep-ph]].

[26] C. Amsler et al. [Particle Data Group], Phys. Lett. B 667, 1 (2008).

[27] V. Baru, C. Hanhart, Yu. S. Kalashnikova, A. E. Kudryavtsev and A. V. Nefediev, Eur. Phys. J. A 44, 93 (2010) [arXiv:1001.0369 [hep-ph]].

[28] C. Chin, R. Grimm, P. Julienne, and E. Tiesinga, Rev. Mod. Phys. 82, 1225 (2010) [arXiv:0812.1496].

[29] S. Godfrey and N. Isgur, Phys. Rev. D 32, 189 (1985).

[30] J. Zeng, J. W. Van Orden and W. Roberts, Phys. Rev. D 52, 5229 (1995) [arXiv:hep$\mathrm{ph} / 9412269]$.

[31] D. Ebert, R. N. Faustov and V. O. Galkin, Phys. Rev. D 67, 014027 (2003) [arXiv:hep$\mathrm{ph} / 0210381]$.

[32] T. Barnes and S. Godfrey, Phys. Rev. D 69, 054008 (2004) [arXiv:hep-ph/0311162].

[33] E. J. Eichten, K. Lane and C. Quigg, Phys. Rev. D 69, 094019 (2004) [arXiv:hep-ph/0401210].

[34] T. Barnes, S. Godfrey and E. S. Swanson, Phys. Rev. D 72, 054026 (2005) [arXiv:hepph/0505002].

[35] E. J. Eichten, K. Lane and C. Quigg, Phys. Rev. D 73, 014014 (2006) [Erratum-ibid. D 73, 079903 (2006)] [arXiv:hep-ph/0511179].

[36] S. F. Radford and W. W. Repko, Phys. Rev. D 75, 074031 (2007) [arXiv:hep-ph/0701117].

[37] M. Suzuki, Phys. Rev. D 72, 114013 (2005) [arXiv:hep-ph/0508258].

[38] C. E. Thomas and F. E. Close, Phys. Rev. D 78, 034007 (2008) [arXiv:0805.3653 [hep-ph]].

[39] N. A. Tornqvist, Z. Phys. C 61, 525 (1994) [arXiv:hep-ph/9310247].

[40] N. A. Tornqvist, Phys. Lett. B 590, 209 (2004) [arXiv:hep-ph/0402237].

[41] X. Liu, Z. G. Luo, Y. R. Liu and S. L. Zhu, Eur. Phys. J. C 61, 411 (2009) [arXiv:0808.0073 [hep-ph]].

[42] E. S. Swanson, Phys. Lett. B 598, 197 (2004) [arXiv:hep-ph/0406080].

[43] D. Gamermann and E. Oset, Phys. Rev. D 80, 014003 (2009) [arXiv:0905.0402 [hep-ph]].

[44] D. Gamermann, J. Nieves, E. Oset and E. Ruiz Arriola, Phys. Rev. D 81, 014029 (2010) [arXiv:0911.4407 [hep-ph]].

[45] M. Beneke, G. Buchalla, M. Neubert and C. T. Sachrajda, Nucl. Phys. B 591, 313 (2000) [arXiv:hep-ph/0006124].

[46] Z. z. Song and K. T. Chao, Phys. Lett. B 568, 127 (2003) [arXiv:hep-ph/0206253].

[47] Z. Z. Song, C. Meng, Y. J. Gao and K. T. Chao, Phys. Rev. D 69, 054009 (2004) [arXiv:hep$\mathrm{ph} / 0309105]$.

[48] M. Beneke and L. Vernazza, Nucl. Phys. B 811, 155 (2009) [arXiv:0810.3575 [hep-ph]].

[49] G. T. Bodwin, X. G. i. Tormo and J. Lee, Phys. Rev. D 81, 114014 (2010) [arXiv:1003.0061 [hep-ph]].

[50] C. Meng, Y. J. Gao and K. T. Chao, arXiv:hep-ph/0506222.

[51] X. Liu and Y. M. Wang, Eur. Phys. J. C 49, 643 (2007). 
[52] H. n. Li and H. L. Yu, Phys. Rev. Lett. 74, 4388 (1995) [arXiv:hep-ph/9409313].

[53] E. J. Eichten and C. Quigg, Phys. Rev. D 52, 1726 (1995) [arXiv:hep-ph/9503356].

[54] J. J. Dudek, R. G. Edwards, N. Mathur and D. G. Richards, Phys. Rev. D 77, 034501 (2008) [arXiv:0707.4162 [hep-lat]].

[55] E. Braaten, M. Kusunoki and D. Zhang, Annals Phys. 323, 1770 (2008) [arXiv:0709.0499 [cond-mat.other]]. 